Alma Mater Studiorum - University OF Bologna

DEPARTMENT OF ELECTRICAL ELECTRONIC AND INFORMATION ENGINEERING - DEI

PhD in Electrical Engineering

XXVI CYCLE

SC 09/E1 - Elettrotecnica

SSD ING-IND/31 - Elettrotecnica

\title{
Peak-to-Peak Output Current Ripple Analysis in Multiphase and Multilevel Inverters
}

CANDIDATE:

Jelena Loncarski
TUTOR:

prof. Gabriele Grandi

PhD COORDINATOR: prof. Domenico Casadei 



\section{Table of contents}

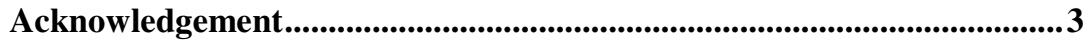

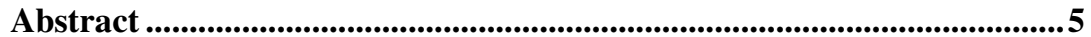

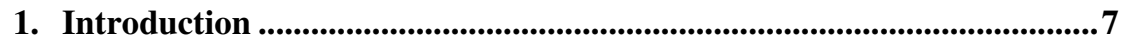

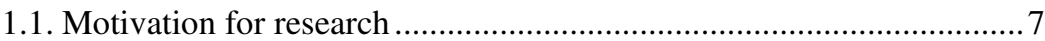

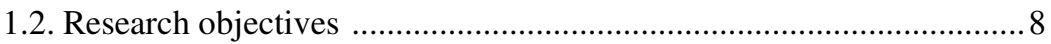

1.3. Outlines and original contribution of the dissertation ....................... 9

2. Analysis of the current ripple in three-phase two-level VSIs.................11

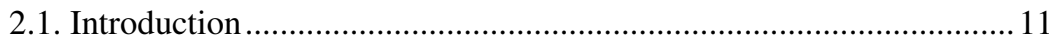

2.1.1. Modulation techniques............................................................ 12

2.2. Output current ripple definition in PWM converters ......................... 15

2.3. Peak-to-peak output current ripple .................................................... 17

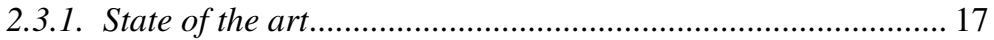

2.3.2. Calculation of the peak-to-peak output current ripple............ 19

2.3.3. Peak-to-peak current ripple diagrams .................................... 24

2.3.4. Maximum and minimum of the current ripple ........................ 24

2.4. Numerical and experimental results ..............................................28

3. Analysis of the output current ripple in multiphase VSIs .....................39

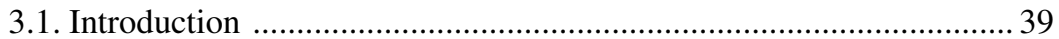

3.1.1. Modulation techniques .......................................................... 40

3.2. Extension of the peak-to-peak output current ripple analysis to

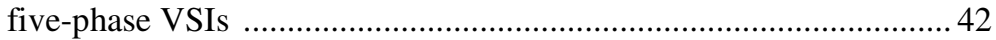

3.2.1. State of the art...................................................................... 42

3.2.2. Calculation of the peak-to-peak output current ripple ............ 43

3.2.3. Peak-to-peak current ripple diagrams .................................. 51

3.2.4. Maximum of the current ripple ……......................................52

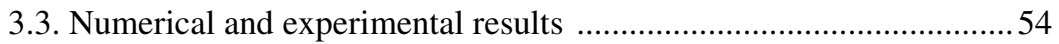

3.4. Extension of the peak-to-peak output current ripple analysis to sevenphase VSIs 
3.4.1. State of the art 61

3.4.2. Calculation of the peak-to-peak output current ripple ............ 61

3.4.3. Peak-to-peak current ripple diagrams .................................. 71

3.4.4. Maximum of the current ripple ............................................. 73

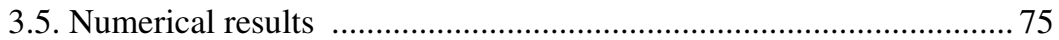

4. Analysis of the output current ripple in multilevel VSIs ........................83

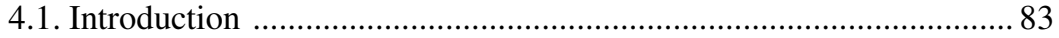

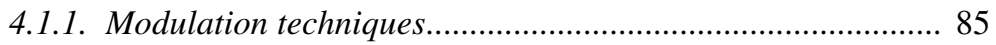

4.2. Extension of the peak-to-peak output current ripple analysis to three-

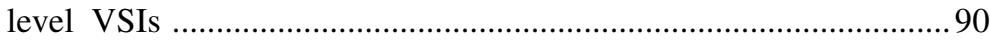

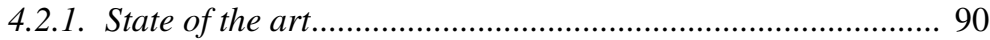

4.2.2. Calculation of the peak-to-peak output current ripple........... 90

4.2.3. Peak-to-peak current ripple diagrams ................................. 105

4.3. Numerical and experimental results ................................................ 107

5. Comparison of the peak-to-peak current ripple ......................................119

5.1. Comparison between different phase number ................................. 119

5.1.1. Review of peak-to-peak current ripple evaluation in

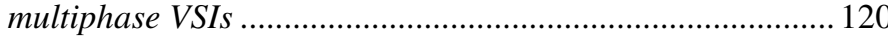

5.1.2. Comparison of the peak-to-peak current ripple diagrams ... 122

5.1.3. Numerical results ........................................................... 125

5.2. Comparison in between different number of level .......................... 128

5.2.1. Review of peak-to-peak current ripple evaluation in

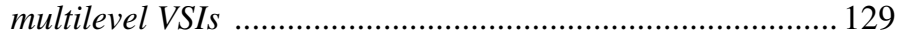

5.2.2. Comparison of the peak-to-peak current ripple diagrams... 131

5.2.3. Numerical results .............................................................. 134

6. Examples of application ................................................................................ 137

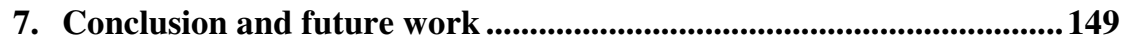

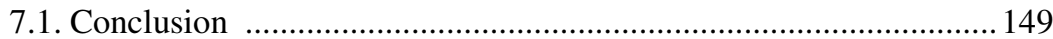

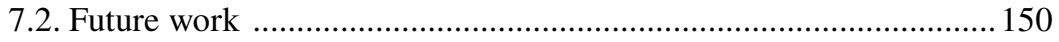




\section{Acknowledgement}

It is my honor to express sincere gratitude to all the people that came across during my research period at University of Bologna and if perhaps have not been listed here, were a great impact on me and my work.

First of all, I would like to thank to my family in Serbia, for all the support and encouragement they provided during all the years of my growth. Without their support I would not have been able to complete my $\mathrm{PhD}$ research work successfully. Furthermore, I would like to thank to all my friends in Serbia for all the care and support they provided me, regardless the distance, and in spite of their own problems due to the decades of difficult situation in Serbia.

My deepest gratitude goes to my advisor, prof. Gabriele Grandi, who has unselfishly helped me with all the issues we have come across during my $\mathrm{PhD}$ research activity. I was very lucky to have had the opportunity to work with an extraordinary person such as himself. Among many difficulties we had to solve, most appreciable was his help from academic point of view. His professional supervision and immense technical knowledge have helped my research to steer in the right direction and framed this thesis. His support and guidance have always given me strength to continue forward. All the merits for the results obtained during my research period should be addressed to him. I am looking forward to extend our collaboration also for the future research work.

Two important persons from the Dept. of Electrical Engineering at UniBO have encouraged my work. First is our $\mathrm{PhD}$ coordinator, prof. Domenico Casadei, to whom I thank for the theoretical and practical concepts he has 
provided to all of us. Second is prof. Claudio Rossi, who has always given practical knowledge and advised me from the point of view of applications.

I wish to express my sincere appreciation for the support I have received during my $\mathrm{PhD}$ course from The Institute of the Advanced Studies (ISA), and especially to the coordinator PhD Barbara Cimatti. Their hospitality meant a lot to me, and it is great to have such an institution at Alma Mater.

I would like to thank also to the group of prof. Emil Levi, Liverpool John Moores University, in particular to $\mathrm{PhD}$ Obrad Dordevic, for the support given to obtain the experimental results, their devoted cooperation and useful adivices.

It has been a priviledge to collaborate with Department for Electrical Engineering, University of Bologna, and I would like to thank to all the colleagues for the work facilities they provided me and their willingness to collaborate.

Bologna, 15.02.2014.

Jelena Loncarski 


\section{Abstract}

Analysis of the peak-to-peak output current ripple amplitude for multiphase and multilevel inverters is presented and discussed in this $\mathrm{PhD}$ thesis. The current ripple is calculated on the basis of the alternating voltage component, and peak-to-peak value is defined by the current slopes and application times of the voltage levels in a switching period. Detailed analytical expressions of peak-to-peak current ripple distribution over a fundamental period are given as function of the modulation index.

For all the cases, reference is made to centered and symmetrical switching patterns, generated either by carrier-based or space vector PWM. By this PWM strategy, the dc bus utilization is maximized in a simple and effective way, and a nearly-optimal modulation is obtained to minimize the current ripple RMS.

Starting from the definition and the analysis of the output current ripple in three-phase two-level inverters, the theoretical developments have been extended to the case of multiphase inverters, with emphasis on the five- and seven-phase inverters. The instantaneous current ripple is introduced for a generic balanced multiphase loads consisting of series RL impedance and ac back emf (RLE). Simplified and effective expressions to account for the maximum of the output current ripple have been defined. The peak-to-peak current ripple diagrams are presented and discussed. Both simulation and experimental results are carried out to prove the validity of the analytical developments.

The analysis of the output current ripple has been extended also to multilevel inverters, specifically three-phase three-level inverters. The optimal modulation has been identified with reference to current ripple RMS 
minimization, as in the previous cases. Also in this case, the current ripple analysis is carried out for a balanced three-phase system consisting of series RL impedance and ac back emf (RLE), representing both motor loads and gridconnected applications. The peak-to-peak current ripple diagrams are presented and discussed. In addition, simulation and experimental results are carried out to prove the validity of the analytical developments.

The cases with different phase numbers and with different number of levels are compared among them, and some useful conclusions have been pointed out. Furthermore, some application examples are given. 


\section{Introduction}

\subsection{Motivation for research}

Three-phase voltage source inverters (VSIs) are widely utilized in ac motor drives, controlled rectifiers, and in general grid-connected applications as dc-ac power conversion devices. In general, the problems related to high-power applications can be overcome by the increase of the number of phases. The increase of the phase number is considered a viable solution and has become more and more used in order to obtain additional degree of freedom. Multiphase motor drives have many advantages over the traditional 3-phase motor drives. Most important are ability to reduce the amplitude and to increase the frequency of torque pulsations, to reduce the rotor harmonic current losses and to lower the dc link current harmonics. Additionally, due to their redundant structure, multiphase motor drives improve the system reliability.

On the other hand, multilevel inverters widely replace the conventional twolevel three-phase voltage source inverters nowadays. Multilevel inverters became more and more popular in last decades, due to the increased power ratings, improved output voltage waveforms, and reduced electromagnetic interference emission. A simple and reliable implementation of multilevel inverter is based on cascaded connection of single-phase inverters (H-bridge), leading to a structure capable of reaching high output voltage amplitudes by using standard low-voltage components. Usually multilevel inverters also feature a high modularity degree due to the fact that each inverter can be seen as module with similar circuit topology, control structure and modulation. Three-level PWM VSIs are the viable converters for many high-power applications, both grid-connected and motor-load. Compared with traditional two-level inverters, three-level inverters have half of the voltage stress on switching devices for the same maximum output voltage and generate lower harmonics for the same switching frequency.

Despite the numerous PWM schemes for multiphase and multilevel VSIs with optimal sinusoidal outputs have been developed, no detailed analysis of the impact of these modulation schemes on the output peak-to-peak current 
ripple amplitude has been reported. This dissertation is devoted towards the definition and the analysis of the output current ripple in multiphase and multilevel inverters.

\subsection{Research objectives}

The main objective of this dissertation is to study and develop the peak-topeak output current ripple for multiphase and multilevel inverters, and to verify the theoretical developments by numerical simulation software and on a real experimental setup. In detail, the objectives are focused towards:

1) Defining the output current ripple in a general case.

2) Development of the three-phase converter with its optimal modulation, in terms of the RMS of the output current, in numerical simulation software Matlab-Simulink. Calculation of the peak-to-peak output current ripple in the case of three-phase inverter. Numerical and experimental verification of the results obtained.

3) Extension of the study of three-phase inverter to the multiphase inverter, with special emphasis on five- and seven-phase inverters, with optimal modulation strategies in numerical simulation software Matlab-Simulink. Extension of the ripple analysis to the case of multiphase inverters. Numerical and experimental verification of the results obtained.

4) Comparison of the output current ripple in multiphase inverters.

5) Extension of the study of three-phase two-level inverter to the three-level inverter, with its optimal modulation, in terms of the RMS of the output current, in numerical simulation software Matlab-Simulink. Extension of the ripple analysis to the case of multilevel inverters. Numerical and experimental verification of the results obtained.

6) Comparison of the output current ripple in two-and three-level inverters.

By achieving the objectives listed above, a significant new knowledge has been produced. This has been evidenced by the already published research papers that have resulted from the thesis, which can be found within cited 
references. Chapters 2-7 contain the original results from the research and therefore represent the main contribution of this work.

\subsection{Outlines and original contribution of the dissertation}

This thesis is divided into following major parts and original contribution is provided by chapters $2-7$ :

\section{Chapter 2: "Analysis of the current ripple in three-phase two-level VSIs"}

This chapter provides survey on optimal three-phase inverter techniques, carrier-based CB-PWM and space vector PWM. Chapter proceeds with the definition of the output current ripple for a general case. Further on, it gives the complete analysis of the peak-to-peak output current ripple in three-phase inverters, with reference to the optimal modulation and three-phase loads, such are both grid-connected and motor loads. The analysis is accompanied with the numerical and experimental results.

\section{Chapter 3: "Analysis of the output current ripple in multiphase VSIs"}

This chapter provides survey on optimal multiphase inverter techniques, carrier-based CB-PWM and space vector PWM, with the emphasis on the fiveand seven-phase inverters. Chapter proceeds with the extension of the analysis of the peak-to-peak output current ripple to five- and seven-phase inverters, with reference to the optimal modulation and multiphase loads such are motor loads. The analysis is accompanied with the numerical and experimental results.

\section{Chapter 4: "Analysis of the output current ripple in multilevel VSIs"}

This chapter provides survey on optimal multilevel inverter techniques, carrier-based CB-PWM and space vector PWM. Chapter proceeds with the extension of the analysis of the peak-to-peak output current ripple in threephase two-level inverters to the three-level inverters, with reference to the optimal modulation and three-phase three-level loads, such are both gridconnected and motor loads. The analysis is accompanied with the numerical and experimental results. 


\section{Chapter 5: "Comparison of the peak-to-peak current ripple"}

This chapter gives the overview on all the developed cases, and proceeds with the comparison within different cases. First presented is the comparison of the output current ripple between cases with different phase numbers, i.e. three-, five-, and seven-phase inverters. Further on, the comparison of the output current ripple in two- and three-level inverters is presented.

\section{Chapter 6: "Examples of application"}

The possible examples of applications are presented in this chapter.

\section{Chapter 7: "Conclusion and future work"}

The main conclusion of this dissertation based on numerical simulation and experimental results are presented. Finally, chapter provides few recommendations for future research work. 


\section{Analysis of the current ripple in three-phase two-level VSIs}

\subsection{Introduction}

Three-phase voltage source inverters (VSIs) are widely utilized in ac motor drives, controlled rectifiers, and in general grid-connected applications as dc-ac power conversion devices. The topology of the three-phase inverter with a generic three-phase $R-L-E M F$ load (RLE) is given in Fig. 2.1. Most of VSI applications employ carrier-based PWM (CB-PWM) control schemes due to their simplicity of implementation, both in analog and digital ways, fixed switching frequency and well defined harmonic spectrum characteristics [1]. Furthermore, switching losses are easier to control with constant switching frequency, and the inverter design can take advantage of an accurate losses calculation. Different types of CB-PWM together with space vector PWM (SV-PWM) are investigated, and optimal solutions are proposed with reference to reduce the current ripple and switching losses [2]-[4]. CB-PWM leads to equivalent switching patterns as SV-PWM by proper zero-sequence modulating signal injection [5].

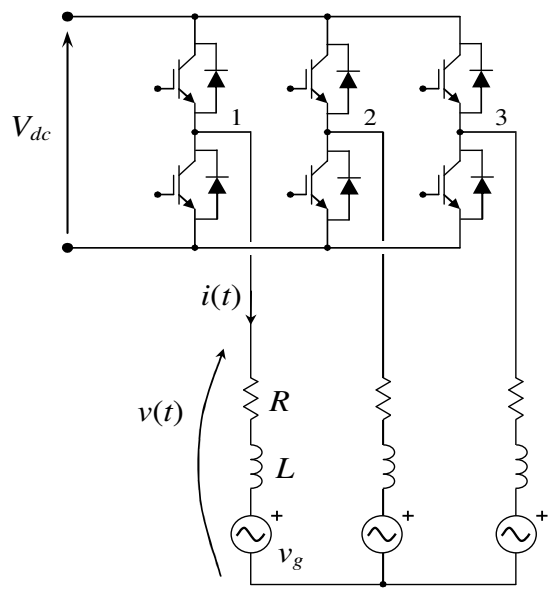

Fig. 2.1. RLE circuit of three-phase inverter. 
A complete analysis of three-phase PWM converter system addressed to switching losses, dc link harmonics, and inverter input/output harmonics is given by [6]. It has been shown that reduction of the output current harmonics in case of continuous PWM can be achieved by proper zero-sequence signal injection. A further reduction of the RMS value of the current ripple is possible in some cases by development of discontinuous PWM schemes, which allow increasing the carrier frequency while maintaining the switching losses as same as continuous PWM. In the case of a loss minimization, two cases are observed in [7], which correspond to the continuous and to the discontinuous modulation as can be seen by comparing the dependencies of the normalized harmonic power losses on the converters modulation index.

\subsubsection{Modulation techniques}

Most common modulation techniques of the three-phase inverter are the carrier-based PWM (CB-PWM) and space vector PWM (SV-PWM).

The traditional method for generating the switching patterns in three-phase VSI with CB-PWM is the use of naturally or regularly sampled sinusoidal waveforms. In general, carrier-based modulation compares a sine wave reference $v^{*}$ with a triangular wave carrier signal. In naturally sampled technique the reference sine wave is allowed to vary during the carrier interval, while in regularly sampled the reference sine wave is held at a constant value during the carrier interval. Regularly sampled modulation can be symmetric, where the reference sine wave is kept constant for the whole carrier period, or asymmetric, where it is resampled in the half of the carrier period. As a result of this comparison the desired inverter switching pattern is realized. In particular, in CB-PWM schemes the three reference phase voltages $v_{a}^{*}, v_{b}^{*}$ and $v_{c}^{*} \quad$ (modulating signals corresponding to the reference voltage vector in SVM), are compared with triangle carrier waveforms as represented in Fig. 2.2, for modulation index $m=0.5$. The frequency of these carriers defines the switching frequency, and their amplitude is the half of the dc-link voltage, eventually in per units. The range of the modulation index defined as $m=$ $V^{*} / V_{d c}$, in this case is $0<m<0.5$.

The zero-sequence injection methods, when the zero-sequence signal is added to the sine wave references, leads to a larger variety of modulating waves. The first studied technique in the literature was the third harmonic 


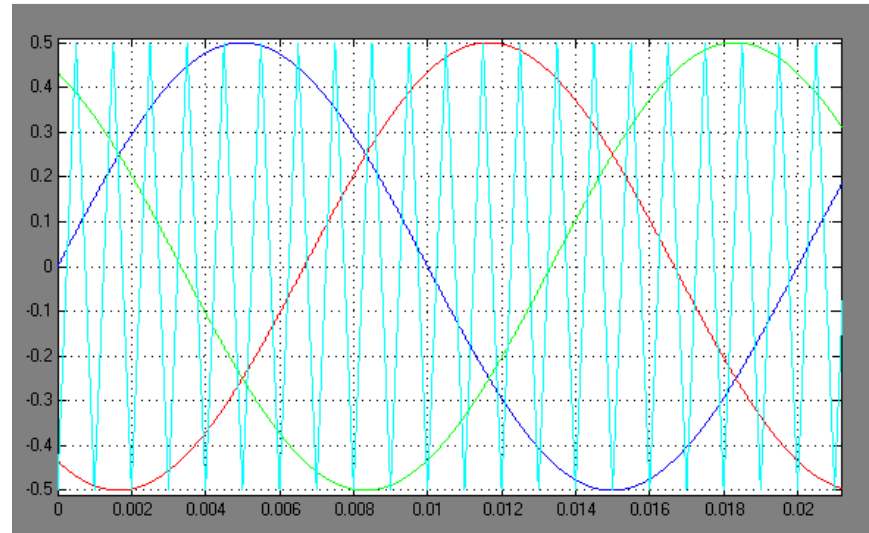

Fig. 2.2. Carrier-based modulation, $m=0.5$.

injection technique, when the $17 \%$ third harmonic component is added to the reference waveforms. Nowadays, most used technique is the so called centered PWM, obtained by centering the reference voltages with $\mathrm{min} / \mathrm{max}$ injection. Common mode signal to be added to the reference voltages $(\mathrm{min} / \mathrm{max}$ injection) is defined for each phase as

$$
v_{c m}=-\frac{1}{2}\left[\max \left(v_{k}^{*}\right)-\min \left(v_{k}^{*}\right)\right], \quad k=\mathrm{a}, \mathrm{b}, \mathrm{c} .
$$

The resulting waveform obtained by adding the common-mode signal to the sine wave references is

$$
\tilde{v}_{k}^{*}=v_{k}^{*}+v_{c m} .
$$

and shown in Fig. 2.3, for modulation index $m=0.577=m_{\max }$. Centered carrier-based modulation allows overmodulation with the respect to the original sine wave PWM, while proved that obtains better outputs spectrum and that is the optimal technique for the output ripple RMS minimization. The modulation limit is $0<m \leq m_{\max }=1 / \sqrt{ } 3$, according to the generalized expression given in [8] for $n$ phases, $m_{\max }=[2 \cos (\pi / 2 n)]^{-1}$.

The SV-PWM of three-phase inverters is based on the determination of application times of active and null inverter voltage vectors $\boldsymbol{v}$ in every switching period $T_{s}$. In case of symmetrical SV-PWM, the sequence is 


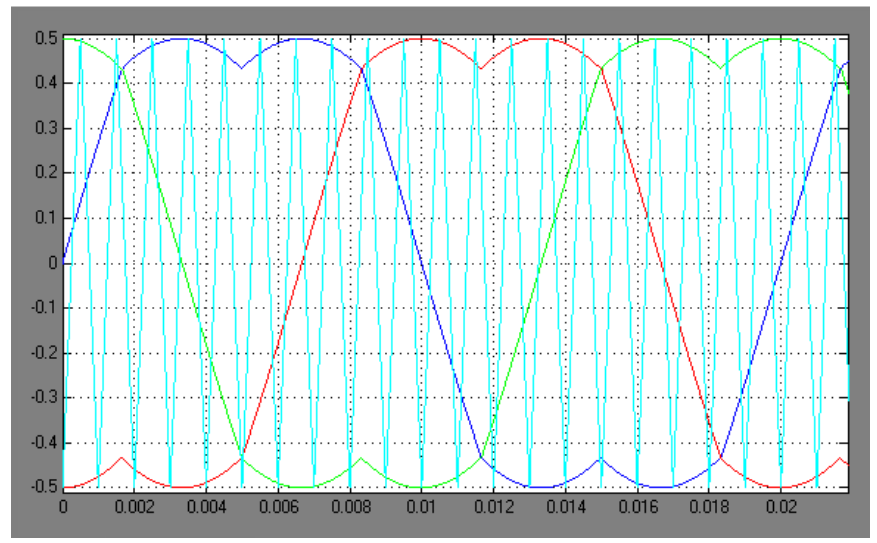

Fig. 2.3. Carrier based modulation with centered reference voltages, $m=0.577=m_{\max }$.

determined in $T_{s} / 2$ and symmetrically repeated in the next half switching period. The principle of SV-PWM in three-phase inverters is summarized with the help of Fig. 2.4, representing the output voltage space vectors corresponding to all possible switch configurations.

The output voltage space vector diagram appears to be a hexagon, consisting of 6 main triangles, numbered I-VI in Fig. 2.4. In two-level inverters the redundancy implies availability of multiple switching states to produce a given voltage vector. There is the only redundancy in zero vector with two possible states, $\{000\}$ and $\{111\}$. The three nearest voltage vectors (NTV) are used to synthesize the reference output voltage space vector $v^{*}$ in each switching period $T_{s}$, being $\boldsymbol{v}^{*}=V^{*} \exp (j \vartheta), V^{*}=m V_{d c}, \vartheta=\omega t, m$ the modulation index, and $V_{d c}$ the dc-bus voltage. The switching sequence begins and ends with the null switching state.

It has been proved that the CB-PWM and SVPWM can be equivalent. By equally sharing the application time of the null voltage vector between the switch configurations 000 and 111 , the centered switching pattern is realized, equivalent to the common-mode injection defined by (2.1) in carrier-based modulation, and nearly-optimal modulation able to minimize the RMS of current ripple is obtained [9]. 


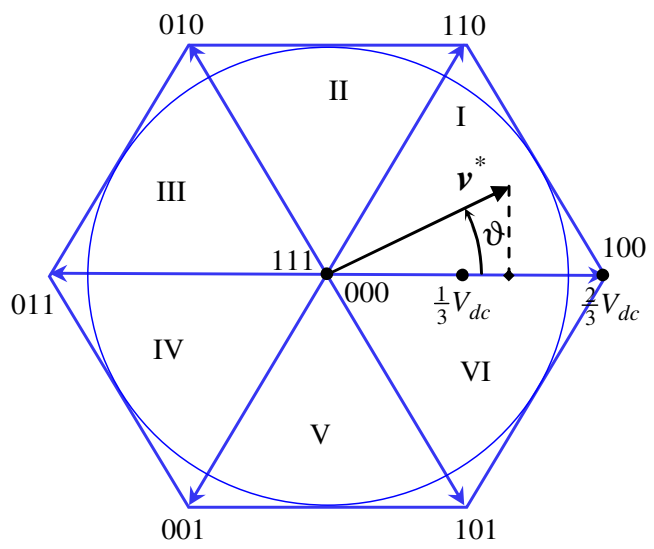

Fig. 2.4. Space vector diagram of inverter output voltage.

\subsection{Output current ripple definition in PWM converters}

Basic circuit scheme for each phase of a balanced $R-L-E M F$ load (RLE) is represented in Fig. 2.5. The voltage equation can be written for each phase as

$$
v(t)=R i(t)+L \frac{d i}{d t}+v_{g}(t) .
$$

Averaging (2.3) over the switching period $T_{s}$ leads to

$$
\bar{v}\left(T_{s}\right)=R \bar{i}\left(T_{s}\right)+L \frac{\Delta i}{T_{s}}+\bar{v}_{g}\left(T_{s}\right),
$$

being:

$$
\Delta i=i\left(T_{s}\right)-i(0) \text {. }
$$

The alternating voltage $\tilde{v}(t)$ is defined as the difference between instantaneous and average voltage components as

$$
\tilde{v}(t)=v(t)-\bar{v}\left(T_{s}\right) .
$$

By introducing (2.3) and (2.4) in (2.6) leads to 


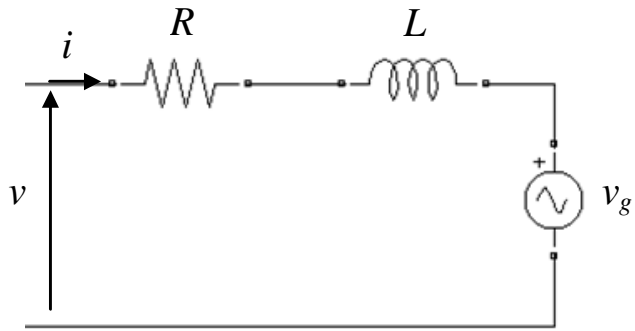

Fig. 2.5. Basic RLE circuit model for one phase.

$$
\tilde{v}(t)=R\left[i(t)-\bar{i}\left(T_{\mathrm{s}}\right)\right]+L\left[\frac{d i}{d t}-\frac{\Delta i}{T_{s}}\right]+\left[v_{g}(t)-\bar{v}_{g}\left(T_{\mathrm{s}}\right)\right] .
$$

The alternating voltage component has amplitude in the order of $V_{d c}$, as shown in Figure 2.6. The first term on the right side in (2.7) accounts for the resistive voltage drop of the current ripple, which is much lower than the rated current. The third (last) term in (2.7) is the deviation of the generator's actual voltage from its average value in the switching period, that is negligible if $v_{g}(t)$ is almost sinusoidal. For these reasons, the expression of alternating voltage component can be simplified to

$$
\tilde{v}(t) \cong L\left[\frac{d i}{d t}-\frac{\Delta i}{T_{s}}\right] .
$$

The current variation in sub-period [0-t], also depicted in Figure 2.6, can be calculated from (2.8) by integrating as

$$
\Delta i(t) \cong \frac{1}{L} \int_{0}^{t} \tilde{v}(t) d t+\frac{t}{T_{s}} \Delta i
$$

Finally, the instantaneous current ripple $\tilde{i}(t)$ can be defined on the basis of (2.9), according to Fig. 2.6

$$
\tilde{i}(t)=\Delta i(t)-\frac{t}{T_{s}} \Delta i \cong \frac{1}{L} \int_{0}^{t} \tilde{v}(t) d t .
$$




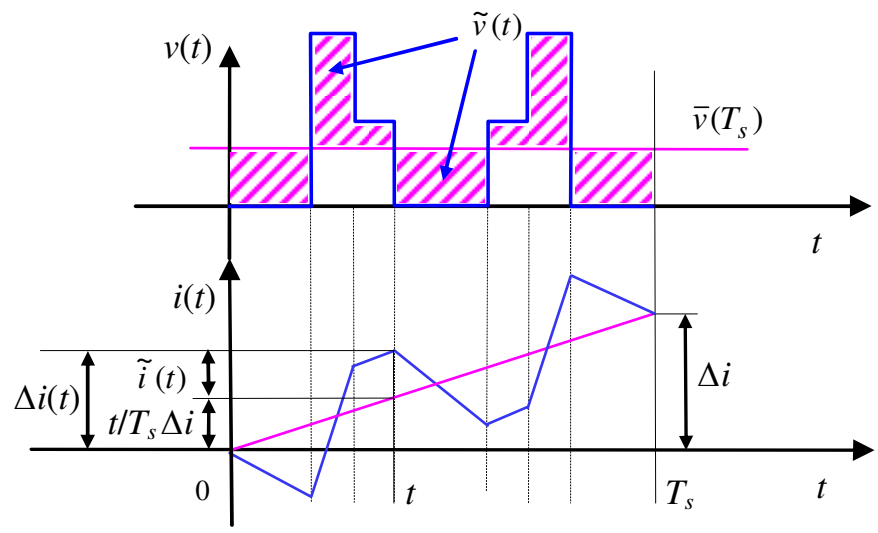

Fig. 2.6. Details of a generic output voltage and current ripple in the switching period.

An example of the behavior of voltage and current in a switching period is given in Fig. 2.6. Note that the current ripple (2.10) corresponds to the difference between the instantaneous current value and its fundamental component.

The peak-to-peak current ripple amplitude is defined as the range of (2.10) in the switching period

$$
\tilde{i}_{p p}=\max \{\tilde{i}(t)\}_{0}^{T_{s}}-\min \{\tilde{i}(t)\}_{0}^{T_{s}} .
$$

\subsection{Peak-to-peak output current ripple}

\subsubsection{State of the art}

In the literature current ripple is studied in the terms of the RMS minimization, rather than the peak-to-peak value. An analytical approach for the analysis of current ripple in SV-PWM controlled induction motor drives for three-phase systems has been presented in [9], where the optimal SVM technique is proposed with calculation of the duty-cycles of the two zero voltage vectors, and on the basis of that the switching pattern of the optimal SVM technique is obtained. Another analysis of current ripple in a sub-period is given in [10], [11], as the error volt-second quantities which are the errors 
between instantaneous applied and reference voltage vectors. Of interest was to study the current ripple RMS over a sub-period and to utilize this knowledge to design hybrid PWM techniques, which reduces the line current distortion. Current ripple RMS is also analyzed in [12], for three-phase inverted feeding passive load. The analysis are conducted on one switching period and of interest was to derive RMS value of output current ripple as function of modulation index.

Newer studies on output current ripple RMS in multiphase drives are presented in [13] and [14], where a five-phase system is considered. Due to existence of more planes (two 2-D planes for five-phase systems), the concept of harmonic flux is used to mitigate initially the dependencies on the equivalent inductances in each plane, where current ripple occurs. It is shown that output current ripple RMS cannot be minimized by injection of fifth harmonic and its odd multiples, but it is also pointed out that, from the practical point of view, differences in current ripple RMS are relatively small considering sinusoidal PWM and SV-PWM. In [14] two SV-PWM techniques are compared (four large vs. two large and two middle vectors) in terms of THD of the current and voltage with established correlations between the flux HDF and the current THD, and squared RMS current ripple. In [15] an attempt to evaluate the output RMS current ripple of a five-phase inverter has been reported, on the basis of polygon load connection and phase variables in the original domain, without the need to use space-vector theory. However, only a single (adjacent) polygon connection has been considered, and the output current-ripple RMS does not represent the total output current ripple [16].

The importance of current ripple amplitude (peak-to-peak) in three-phase systems is recognized in [17], where ripple is analyzed by introducing Thévenin equivalent circuit of the 8 different voltage vectors. In general, the evaluation of current ripple allows adjusting the switching frequency for certain ripple requirements [18]. As another example of application, the knowledge of the peak-to-peak current ripple distribution can be useful to determine the output voltage distortion due to the inverter dead-time in case of output currents with high ripple, by determining the multiple zero-crossing interval [19]. This aspect will be examined in more details in further chapter which deals with applications. Furthermore, the peak-to-peak current ripple amplitude, in addition to the fundamental current component, is useful to 
determine the absolute current peak, affecting the thresholds of protection systems and the design of power components

\subsubsection{Calculation of the peak-to-peak output current ripple}

The output current ripple affects the noise and loss in both the PWM converter and the load, and it should be minimized to improve the system efficiency. In general, the impact of PWM techniques on the current ripple should be understood to further develop the modulation strategy. The analysis of three-phase inverters by the space vector transformation leads to better understanding and more simple calculation of voltage levels and corresponding application times. In the following analysis is considered the centered PWM, as described in Section 2.1.1. The reference is made to a generic balanced RLE load, representing both grid-connected and motor loads.

As result of the SV-PWM, for each phase, the average of the inverter output voltage $\bar{v}\left(T_{s}\right)$ corresponds to the reference voltage $v^{*}$. In the case of sinusoidal balanced output voltages supplying a balanced load, the reference output voltage vector is $v^{*}=m V_{d c} \exp (j \vartheta)$. Reference is made to Fig. 2.7. In this case, $\mathrm{SV}$ modulation is quarter-wave symmetric, so it can be analyzed in the range $\left[0,90^{\circ}\right]$ of the phase angle $\vartheta=\omega t$. In particular, the two ranges $0 \leq \vartheta \leq 60^{\circ}$, and $60^{\circ} \leq \vartheta \leq 90^{\circ}$ can be considered, as depicted in Fig. 2.7.

In the range $0 \leq \vartheta \leq 60^{\circ}$, the application times are:

$$
\begin{array}{rr}
t_{1}=m \sqrt{3} \frac{T_{s}}{2} \sin \left(60^{\circ}-\vartheta\right), & \{100\}, \\
t_{2}=m \sqrt{3} \frac{T_{s}}{2} \sin \vartheta, & \{110\}, \\
t_{0}=\frac{T_{s}}{2}-\left(t_{1}+t_{2}\right)=\frac{T_{s}}{2}\left[1-\sqrt{3} m \sin \left(60^{\circ}+\vartheta\right)\right], & \left\{\begin{array}{l}
000 \\
111
\end{array}\right\} .
\end{array}
$$

In the range $60^{\circ} \leq \vartheta \leq 90^{\circ}$, the application times are:

$$
\begin{array}{ll}
t_{1}=m \sqrt{3} \frac{T_{s}}{2} \sin \left(120^{\circ}-\vartheta\right), & \{110\}, \\
t_{2}=m \sqrt{3} \frac{T_{s}}{2} \sin \left(\vartheta-60^{\circ}\right), & \{010\},
\end{array}
$$




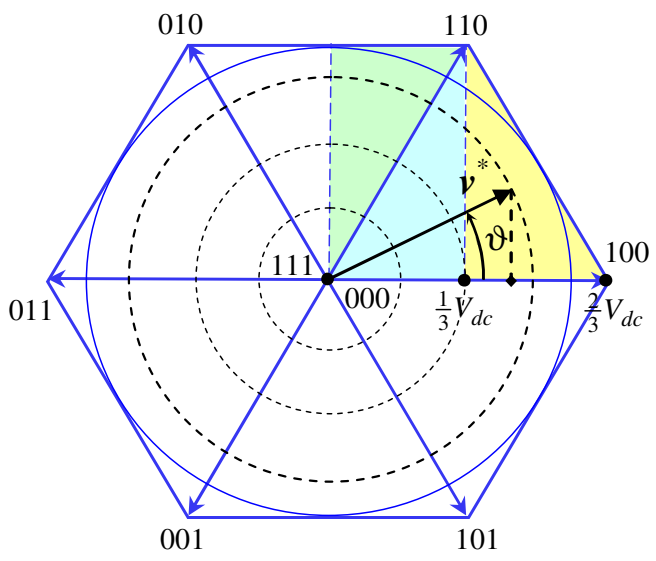

Fig. 2.7. Space vector diagram of inverter output voltage with six sectors and the three identified triangles. Dashed circles represent $m=1 / 6,1 / 3,1 / 2$.

$$
t_{0}=\frac{T_{s}}{2}-\left(t_{1}+t_{2}\right)=\frac{T_{s}}{2}[1-\sqrt{3} m \sin \vartheta],\left\{\begin{array}{l}
000 \\
111
\end{array}\right\} .
$$

Due to the symmetry among the three phases in the considered case of sinusoidal balanced currents, only the first phase is examined in the following analysis. In terms of space vectors, the variables of the first phase are given by the projection of the corresponding space vectors on the real axes. In particular, the average output voltage is given by

$$
\bar{v}\left(T_{s}\right)=v^{*}=\operatorname{Re}\left\{\mathbf{v}^{*}\right\}=V^{*} \cos \vartheta=V_{d c} m \cos \vartheta .
$$

By introducing (2.18) in (2.6), the alternative component of inverter output voltage of the first phase can be written as

$$
\tilde{v}(t)=\left[S_{1}-\frac{1}{3}\left(S_{1}+S_{2}+S_{3}\right)\right] V_{d c}-m V_{d c} \cos \vartheta,
$$

being $S_{k}=[0,1]$ the switch state of the $k$-th inverter leg. In order to evaluate the current ripple in the whole phase angle range $0<\vartheta<90^{\circ}$, the three different triangles depicted in Fig. 2.7 must be separately considered. 


\section{1) Evaluation in the range $0 \leq \vartheta \leq 60^{\circ}$}

Considering the first sector of the hexagon, $0 \leq \vartheta \leq 60^{\circ}$, two different cases can be distinguished, $0 \leq m \cos \vartheta \leq 1 / 3$ and $m \cos \vartheta \geq 1 / 3$, corresponding to the two colored triangular regions in Fig. 2.7.

For modulation index $0 \leq m \cos \vartheta \leq 1 / 3$, the current ripple $\tilde{i}$ and its peakto-peak value $\tilde{i}_{p p}$ are depicted in Fig. 2.8 , together with the instantaneous output voltage $v(t)$. According to Fig. 2.8, $\tilde{i}_{p p}$ can be evaluated by (2.10), (2.11), and (2.19), considering application interval $t_{0}$ (light blue area in Fig. 2.7), leading to

$$
\tilde{i}_{p p}=\frac{1}{L}\left\{V_{d c} m \cos \vartheta t_{0}\right\} .
$$

The case of $1 / 3 \leq m \cos \vartheta \leq 1 / \sqrt{ } 3$ is depicted in Fig. 2.9. In this case $\tilde{i}_{p p}$ can be evaluated considering the application intervals $t_{0} / 2$ and $t_{2}$ (yellow area in Fig. 2.7), leading to

$$
\tilde{i}_{p p}=\frac{2}{L}\left\{V_{d c} m \cos \vartheta \frac{t_{0}}{2}+\left(V_{d c} m \cos \vartheta-\frac{V_{d c}}{3}\right) t_{2}\right\} .
$$

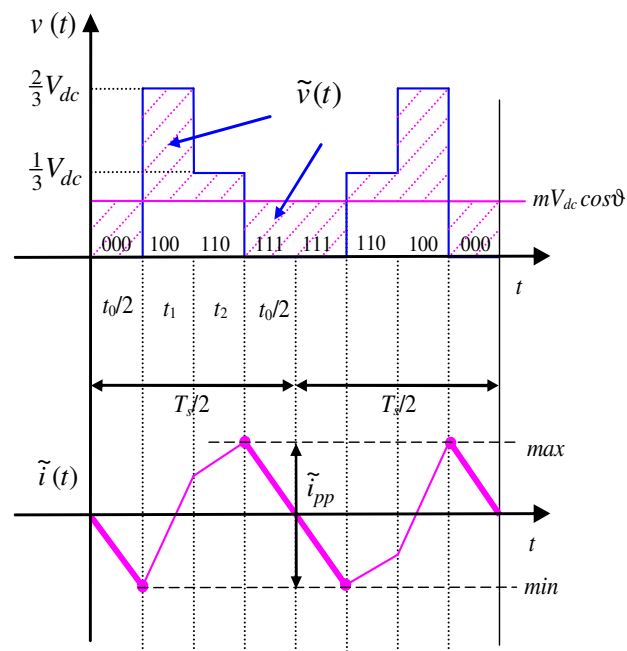

Fig. 2.8. Output voltage and current ripple in one switching period $\left(0 \leq \vartheta \leq 60^{\circ}, 0 \leq m \cos \vartheta \leq 1 / 3\right)$. 


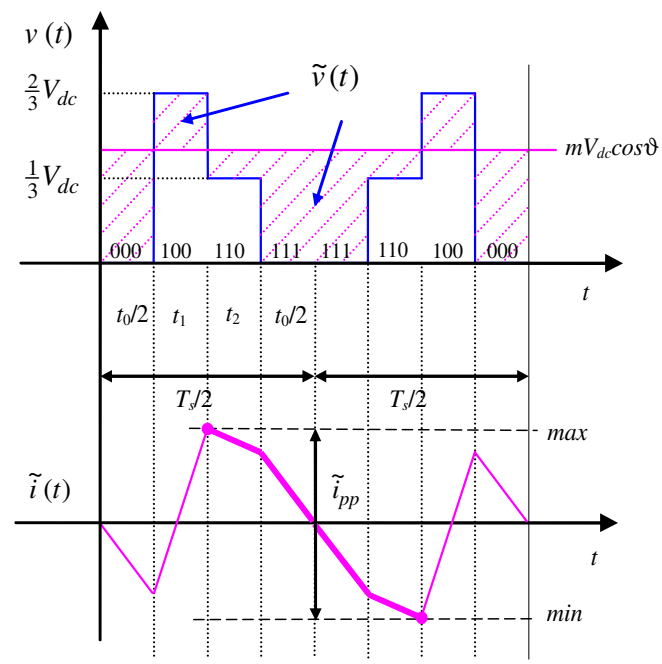

Fig. 2.9. Output voltage and current ripple in one switching period $\left(0 \leq \vartheta \leq 60^{\circ}, m \cos \vartheta>1 / 3\right)$.

For both cases (2.20) and (2.21), introducing the expressions for application intervals $t_{0} / 2$ and $t_{2},(2.14)$ and (2.13), peak-to-peak current ripple can be written as

$$
\tilde{i}_{p p}=\frac{V_{d c} T_{s}}{2 L} r(m, \vartheta),
$$

being $r(m, \vartheta)$ the normalized peak-to-peak current ripple amplitude given by

$$
\begin{aligned}
r(m, \vartheta)= & m \cos \vartheta\left[1-\sqrt{3} m \sin \left(\vartheta+60^{\circ}\right)\right], \quad 0 \leq m \cos \vartheta \leq \frac{1}{3}, \\
r(m, \vartheta)= & m\left\{\cos \vartheta\left[1-\sqrt{3} m \sin \left(\vartheta+60^{\circ}\right)\right]+\right. \\
& \left.+2 \sqrt{3} \sin \vartheta\left(m \cos \vartheta-\frac{1}{3}\right)\right\}, \quad \frac{1}{3} \leq m \cos \vartheta \leq \frac{1}{\sqrt{3}} .
\end{aligned}
$$




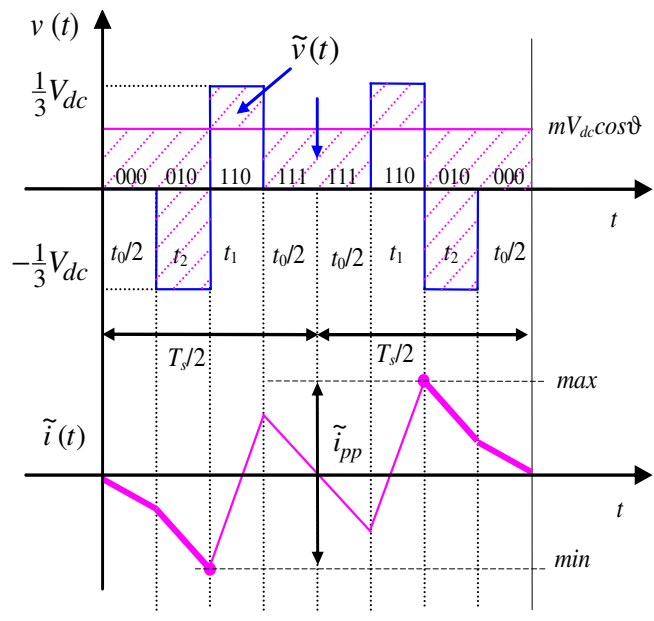

Fig. 2. 10. Output voltage and current ripple in one switching period $\left(60^{\circ} \leq \vartheta \leq 90^{\circ}\right)$.

2) Evaluation in the range $60^{\circ} \leq \vartheta \leq 90^{\circ}$

Considering the third triangle depicted in Fig. 2.7, (green area), $60^{\circ} \leq \vartheta \leq$ $90^{\circ}$, the peak-to-peak current ripple amplitude can be determined considering the application intervals $t_{0} / 2$ and $t_{2}$ for all modulation indexes, according to Fig. 2.10. Introducing (2.19) in (2.10) and (2.11) leads to

$$
\tilde{i}_{p p}=\frac{2}{L}\left\{V_{d c} m \cos \vartheta \frac{t_{0}}{2}+\left[V_{d c} m \cos \vartheta+\frac{1}{3} V_{d c}\right] t_{2}\right\} .
$$

Considering the expressions for application intervals $t_{0}$ and $t_{2},(2.17)$ and (2.16), normalized current ripple amplitude is

$$
r(m, \vartheta)=m\left\{\frac{1}{\sqrt{3}} \sin \vartheta-3 m \cos ^{2} \vartheta\right\} .
$$




\subsubsection{Peak-to-peak current ripple diagrams}

In order to show the behavior of the peak-to-peak current ripple amplitude in the fundamental period for all the possible cases, in Figs. 2.11 and 2.12 is represented the normalized function $r(m, \vartheta)$ defined by (2.22). Figure 2.11. shows $r(\vartheta)$ for $m=1 / 6,1 / 3$, and $1 / 2$, corresponding to the dashed circles in Fig. 2.7. Since symmetric PWM modulation is considered, ripple is symmetric as well, so the ripple envelope corresponds to the half of $\tilde{i}_{p p}$. The two regions ( 0 $\leq \vartheta \leq 60^{\circ}$ and $60^{\circ} \leq \vartheta \leq 90^{\circ}$ ) can be distinguished for $m=1 / 6$ and $1 / 3$. All the three regions are visible for $m=1 / 2$, according to Fig. 2.7.

Figure 2.12 shows the colored map of $r(m, \vartheta)$ in the $1^{\text {st }}$ quadrant within the modulation limits. The ripple amplitude is almost proportional to $m$ in the neighborhoods of $m=0$. Phase angle with minimum ripple can be identified, that is $\vartheta \approx 50^{\circ} \div 60^{\circ}$, and a phase angle with maximum ripple, that is $\vartheta=90^{\circ}$. These aspects are further developed in the following section.

\subsubsection{Maximum and minimum of the current ripple}

In order to estimate current ripple amplitude in the whole fundamental period, the maximum and the minimum of the current ripple can be evaluated. For this purpose, four relevant points can be noticed in Figs. 2.11 and 2.12, as mentioned above: two local maxima, for $\vartheta=0$ and for $\vartheta=90^{\circ}$, and two local minima for $\vartheta=60^{\circ}$ and for $m \cos \vartheta=1 / 3$, i.e., around $\vartheta=50^{\circ}$.

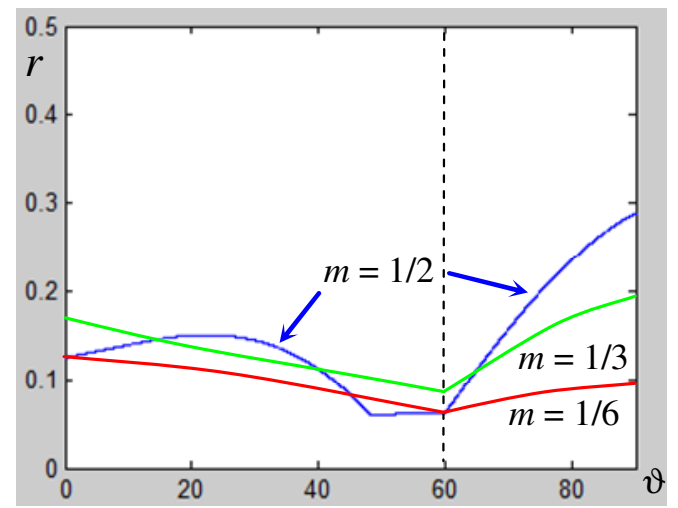

Fig. 2.11. Normalized peak-to-peak current ripple amplitude $r(m, \vartheta)$ for three modulation indexes, $m=1 / 6,1 / 3$, and $1 / 2$, in the phase angle range $\left[0,90^{\circ}\right]$. 


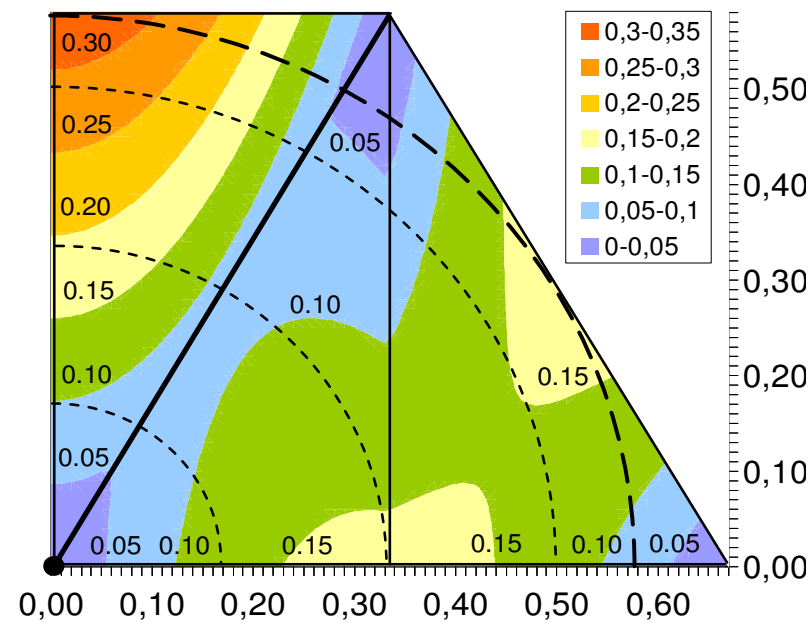

Fig. 2.12. Map of the normalized peak-to-peak current ripple amplitude $r(m, \vartheta)$.

To determine the local maxima, $\vartheta=0$ can be set in (2.23), and $\vartheta=90^{\circ}$ in (2.26), leading to the following global maximum

$$
r^{\max }(m)=\max \left\{m\left[1-\frac{3}{2} m\right], \frac{1}{\sqrt{3}} m\right\} .
$$

The intersection between the two local maxima gives the border value of modulation index

$$
m\left[1-\frac{3}{2} m\right]=\frac{1}{\sqrt{3}} m,
$$

leading to $m \approx 0.282$. Finally, combining (2.27) and (2.28), the maximum of normalized current ripple is

$$
r^{\max }(m)= \begin{cases}m\left[1-\frac{3}{2} m\right] & \text { for } m<0.282, \\ \frac{1}{\sqrt{3}} m & \text { for } m>0.282 .\end{cases}
$$


In order to determine the local minima, $\vartheta=60^{\circ}$ can be set in either (2.23) or (2.26), and $m \cos \vartheta=1 / 3$ in (2.23). The expression for the global minimum of normalized current ripple is

$$
r^{\min }(m)=\min \left\{m\left[\frac{1}{2}-\frac{3}{4} m\right], \frac{1}{6}\left[1-\sqrt{3 m^{2}-\frac{1}{3}}\right]\right\} .
$$

The intersection between the two local minima gives the border value of the modulation index

$$
m\left[\frac{1}{2}-\frac{3}{4} m\right]=\frac{1}{6}\left[1-\sqrt{3 m^{2}-\frac{1}{3}}\right],
$$

leading to $m \approx 0.479$. Finally, combining (2.30) and (2.31), the minimum of normalized current ripple is

$$
r^{\min }(m)= \begin{cases}m\left[\frac{1}{2}-\frac{3}{4} m\right] & \text { for } m<0.479 \\ \frac{1}{6}\left[1-\sqrt{3 m^{2}-\frac{1}{3}}\right] & \text { for } m>0.479 .\end{cases}
$$

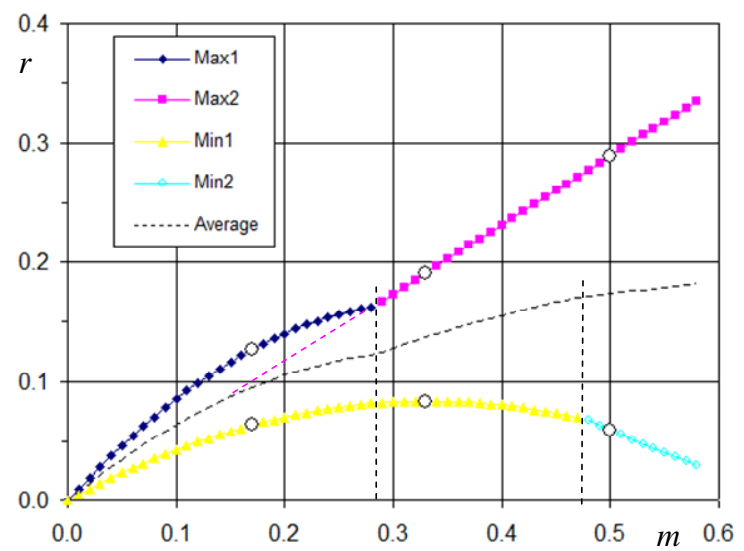

Fig. 2.13. Maximum and minimum of normalized peak-to-peak current ripple amplitude as function of modulation index. 
The composition of the two local minima and maxima are given in Fig. 2.13, leading to global maximum and minimum. The approximated average value is presented with dashed line. The white dots represent the specific points for $m=$ $1 / 6,1 / 3$, and $1 / 2$, displayed in Fig. 2.11, and further examined in simulations and experiments. It can be noted that the maximum function is almost linear for every modulation index, strictly for $m>0.282$. Then, on the basis of (2.22) and (2.29), a simplified expression for maximum of peak-to-peak current ripple amplitude is obtained as

$$
\tilde{i}_{p p}{ }^{\max } \cong \frac{V_{d c} T_{s}}{2 \sqrt{3} L} m
$$

The exact average of the normalized peak-to-peak current ripple, calculated for the more points, is presented in Fig. 2.14. It shows very similar behavior to the approximated one shown in Fig. 2.13. Almost linear behavior can be observed for the modulation indexes $m>0.282$.

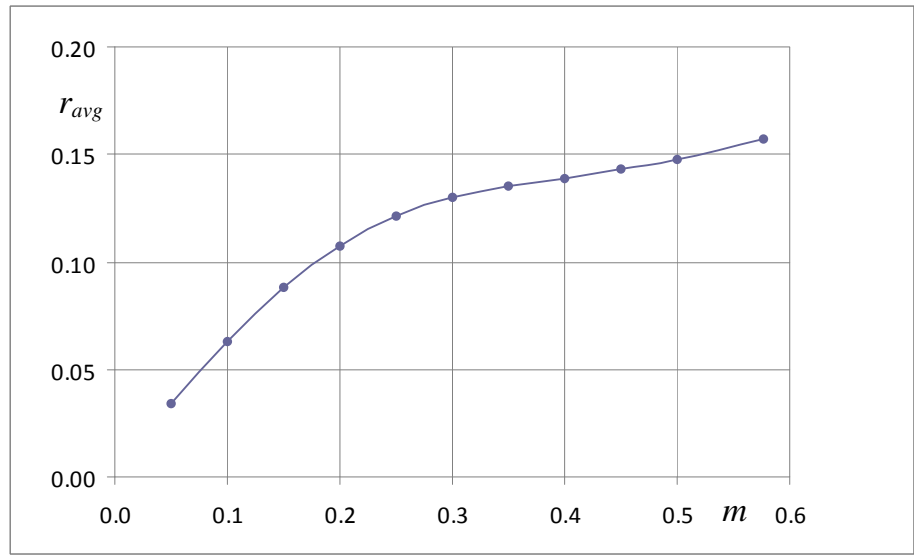

Fig. 2.14. The average of the normalized peak-to-peak current ripple amplitude as function of modulation index. 


\subsection{Numerical and experimental results}

In order to verify the analytical developments proposed in previous sections, numerical simulations and corresponding experimental tests are carried out. Circuit simulations are performed by Sim-PowerSystems of Matlab considering three-phase inverter with ideal switches connected to sinusoidal voltage sources by RL impedance, having $R=4 \Omega$ and $L=24 \mathrm{mH}$. Power transistors have been modeled with ideal switches in order to have a very precise matching with the analytical developments. By introducing non idealities in simulations, such as commutation times, voltage drops, parasitic elements, dead-times etc, leads to output voltage distortion with additional harmonics [20], making more difficult the validation of the proposed theoretical approach. This topology could represent a general three-phase inverter with either motor or grid-connected loads. In all simulations the fundamental frequency is set to $50 \mathrm{~Hz}$, the switching frequency $1 / T_{s}$ is $2.1 \mathrm{kHz}$, and the dc voltage supply $V_{d c}$ is $600 \mathrm{~V}$. A centered symmetrical carrier-based PWM technique, equivalent to centered space vector modulation, is considered in both simulations and experiments, being easier for the implementation.

The experimental setup with custom-built two-level three-phase inverter is shown in Fig. 2.15. Power switches are Infineon FS50R12KE3 IGBT pack. DSpace ds1006 hardware has been employed for the real-time implementation of the algorithm. Dc-bus voltage $\left(V_{d c}\right)$ is set to $600 \mathrm{~V}$ by Sorensen SGI 600/25 dc supply. Switching frequency was set to $2.1 \mathrm{kHz}$ and the inverter's dead time of $6 \mu$ s (not compensated) is implemented in the hardware. Fundamental frequency was kept at $50 \mathrm{~Hz}$ for easier comparison with analytical developments. The nearly-optimal centered space vector PWM is implemented.

The load was a three-phase induction motor (mechanically unloaded). Main motor parameters (all referred to stator) are: stator resistance $R_{s}=2.4 \Omega$, rotor resistance $R_{r}^{\prime}=1.6 \Omega$, stator leakage inductance $L_{l s}=12 \mathrm{mH}$, rotor leakage inductance $L_{l r}{ }^{\prime}=12 \mathrm{mH}$, magnetizing inductance $L_{m}=300 \mathrm{mH}$, and pole pairs $p=2$. According to the model of induction motor for higher order harmonics, which are determining the current ripple, the equivalent inductance $L=L_{l s}+$ $L_{l r}{ }^{\prime}=24 \mathrm{mH}$ is considered for the ripple evaluation. 

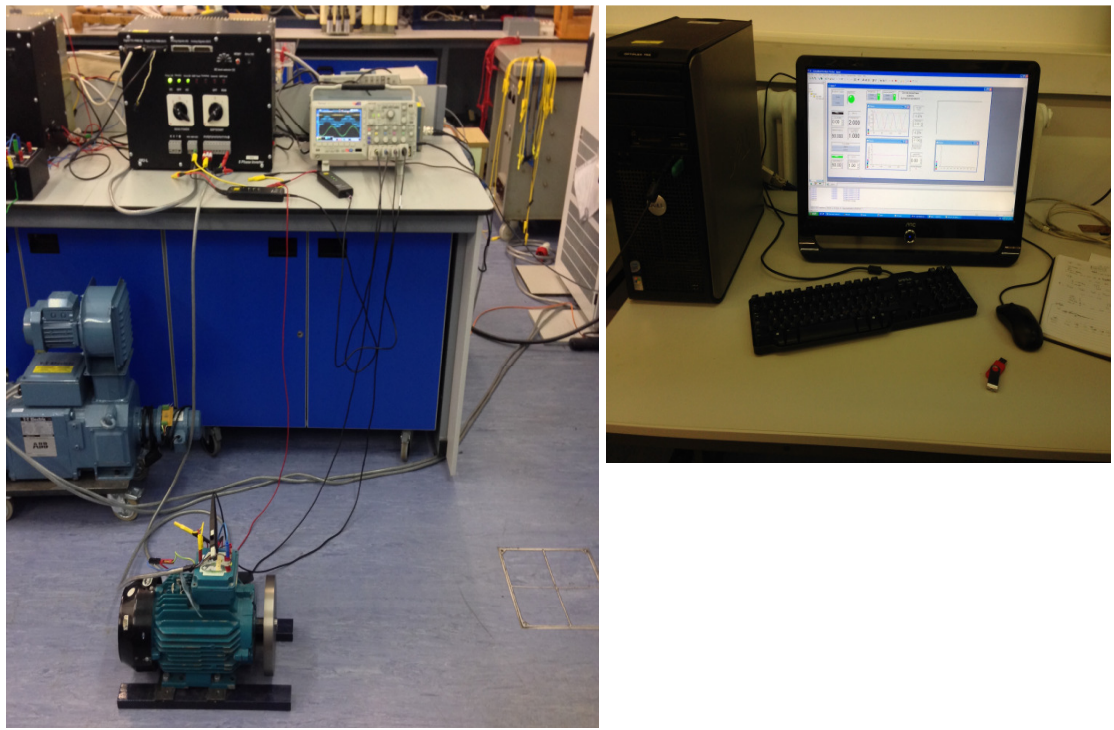

Fig. 2.15. Experimental setup

Tektronix oscilloscope MSO2014 with current probe TCP0030 was used for measurements, and the built-in noise filter (cut-off frequency $f_{c}=600 \mathrm{kHz}$ ) was applied. The instantaneous current ripple $\tilde{i}$ in both simulations and experiments is calculated as the difference between the instantaneous current and its fundamental component, i.e.

$$
\tilde{i}(t)=i(t)-I_{\text {fund }}(t) .
$$

The three-phase system is well balanced and the first phase is selected for further analysis, as in analytical developments. Different values of $m$ have been investigated $\left(1 / 6,1 / 4,1 / 3,1 / 2\right.$, and $\left.1 / \sqrt{ } 3=m_{\max }\right)$.

In Figs. 2.16, 2.18, 2.20, 2.22, and 2.24 is shown the current ripple obtained with simulations (a) and experiments (b), for different modulation indexes, respectively. Comparison is made with the half of the peak-to-peak current ripple, $\tilde{i}_{p p} / 2$. Positive and negative envelopes (blue traces) are determined by the equations presented in Section 2.3.2. The corresponding experimental results are shown as gray traces. 
In Figs. 2.17, 2.19, 2.21, 2.23, and 2.25 is depicted the instantaneous output current obtained in simulations (a) and experiments (b) with different modulation indexes, respectively. Current is shown with the calculated upper/lower ripple envelope, depicted in blue colors. The corresponding experimental results are shown in grey traces.

The values of the modulation index cover all possible sub-cases (different colored regions in Fig. 2.6). The agreement is almost good in the whole fundamental period. Small mismatches can be observed in the experimental current ripple, especially for the higher modulation indexes. These mismatches were probably introduced by the dead-times which in this case were not compensated, but this point can be further examined in the future work. 
(a)

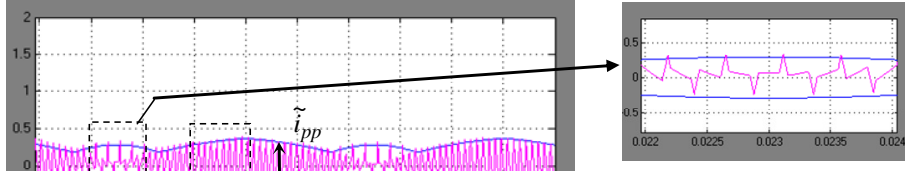

(b)

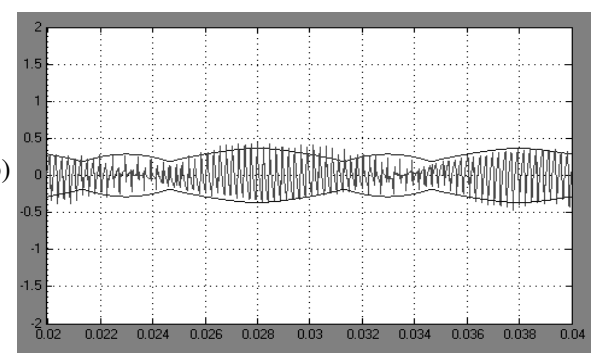

Fig. 2.16. Current ripple (pink), $m=1 / 6$, and evaluated peak-to-peak envelope (blue) for one fundamental period: (a) simulation, with details, and (b) experimental results.

(a)

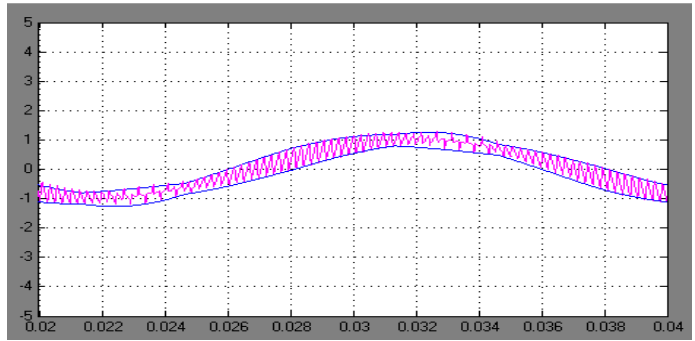

(b)

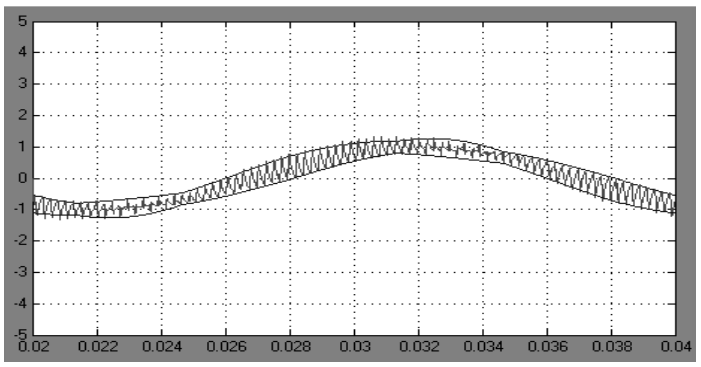

Fig. 2.17. Instantaneous output current (pink) with calculated current envelopes (blue traces) for $m=1 / 6$ : (a) simulated, (b) experimental results. 
(a)

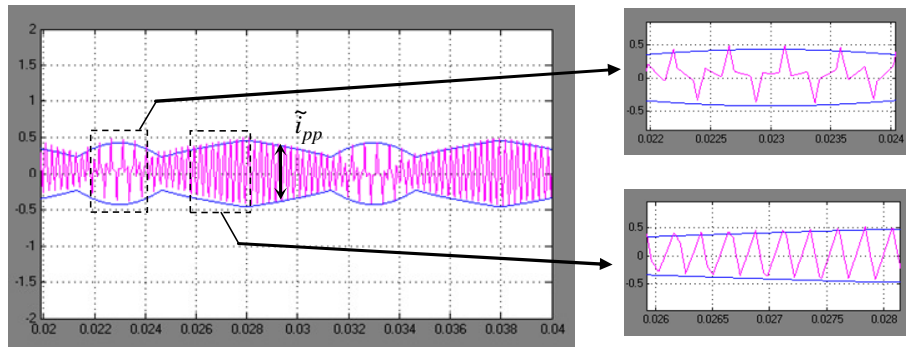

(b)

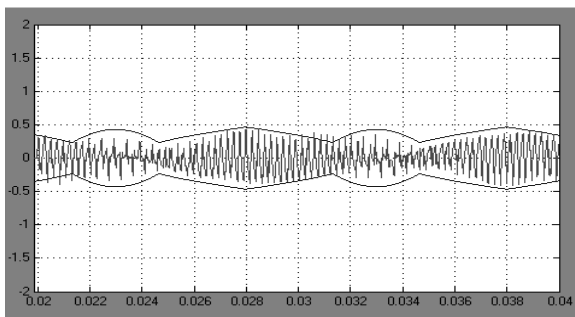

Fig. 2.18. Current ripple (pink), $m=1 / 4$, and evaluated peak-to-peak envelope (blue) for one fundamental period: (a) simulation, with details, and (b) experimental results.

(a)

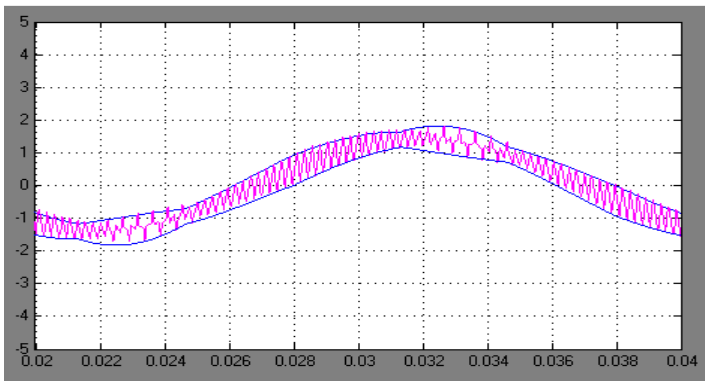

(b)

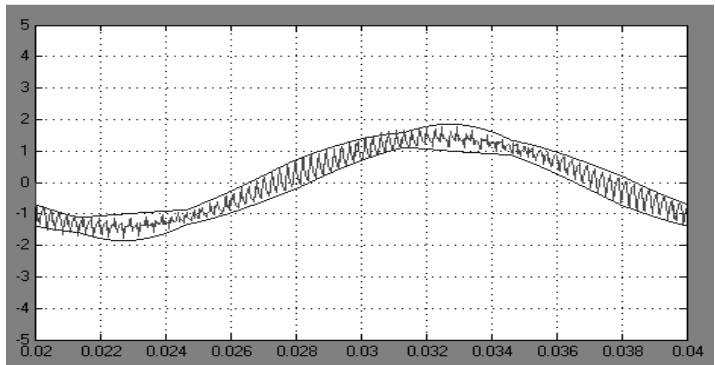

Fig. 2.19. Instantaneous output current (pink) with calculated current envelopes (blue traces) for $m=1 / 4$ : (a) simulated, (b) experimental results. 
(a)

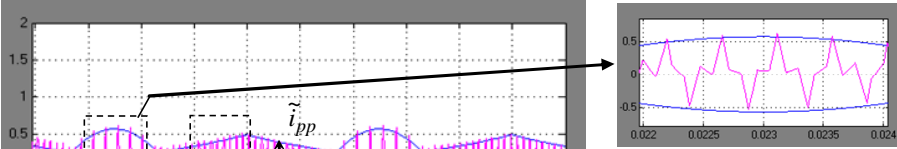

(b)

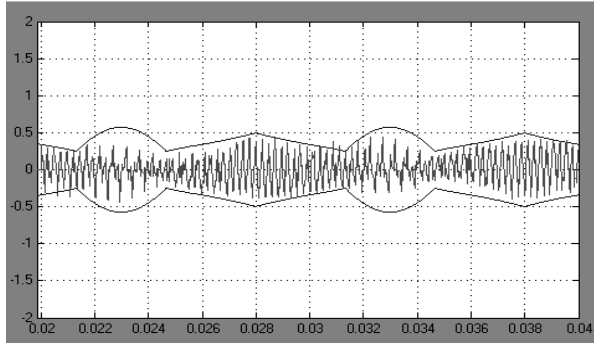

Fig. 2.20. Current ripple (pink), $m=1 / 3$, and evaluated peak-to-peak envelope (blue) for one fundamental period: (a) simulation, with details, and (b) experimental results.

(a)

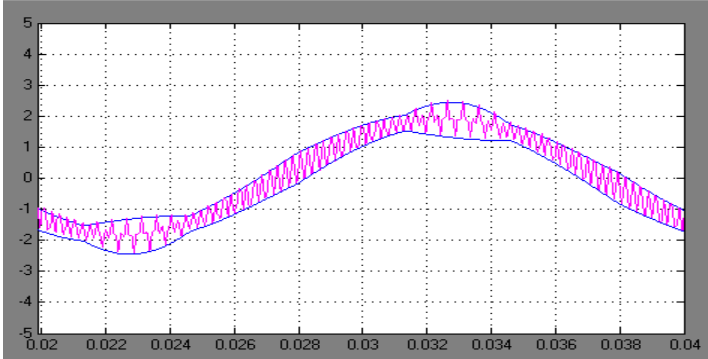

(b)

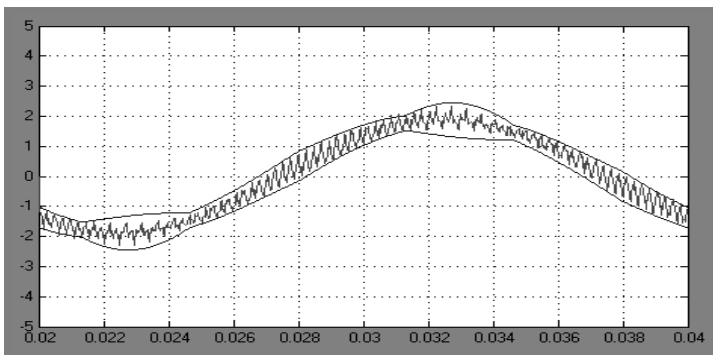

Fig. 2.21. Instantaneous output current (pink) with calculated current envelopes (blue traces) for $m=1 / 3$ : (a) simulated, (b) experimental results. 
(a)

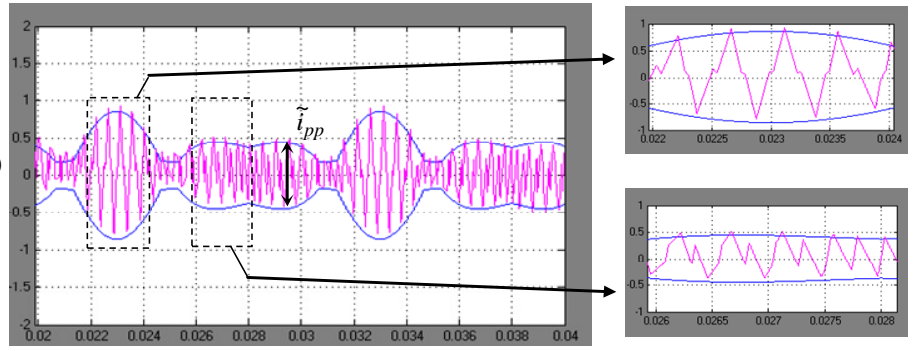

(b)

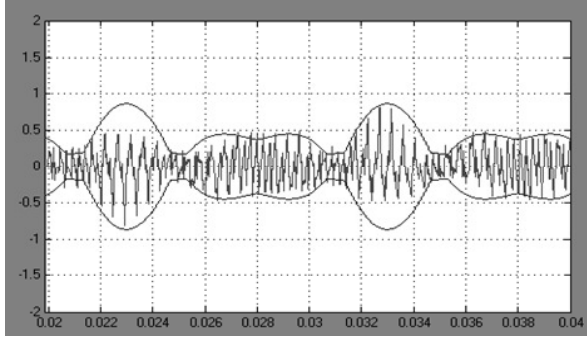

Fig. 2.22. Current ripple (pink), $m=1 / 2$, and evaluated peak-to-peak envelope (blue) for one fundamental period: (a) simulation, with details, and (b) experimental results.

(a)

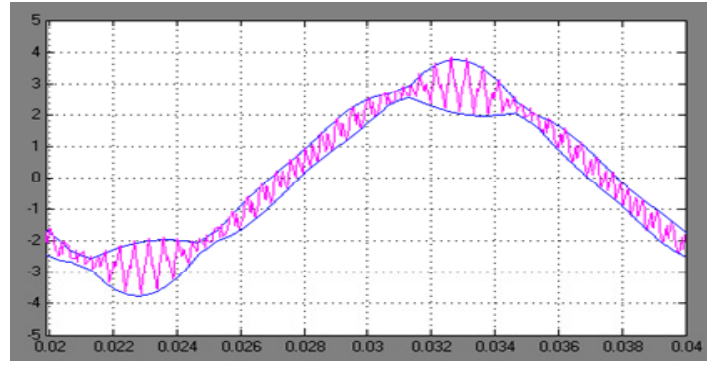

(b)

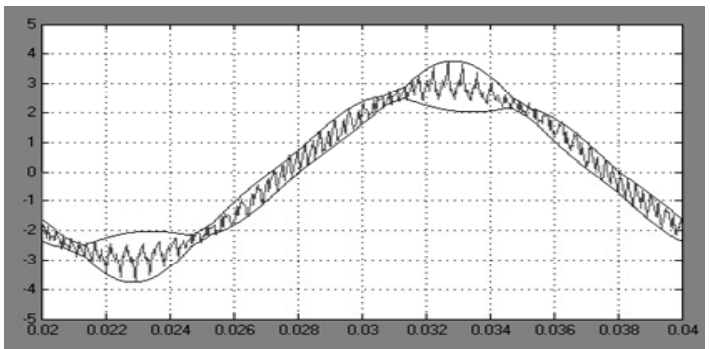

Fig. 2.23. Instantaneous output current (pink) with calculated current envelopes (blue traces) for $m=1 / 2$ : (a) simulated, (b) experimental results. 
(a)

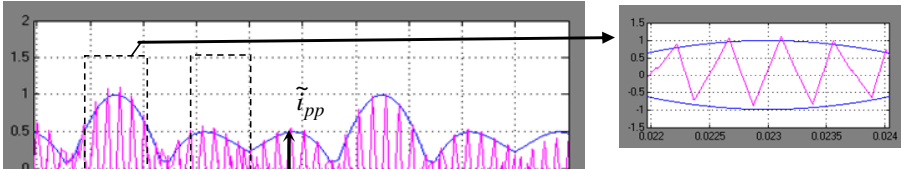

(b)

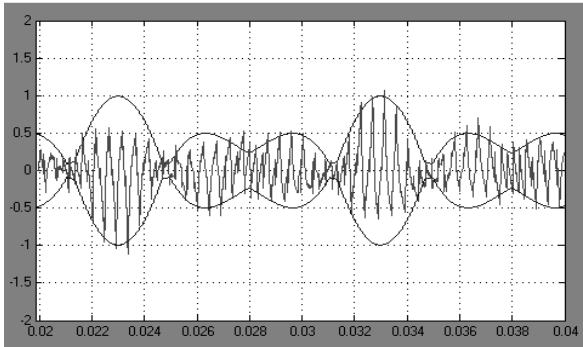

Fig. 2.24. Current ripple (pink), $m=1 / \sqrt{3}=m_{\max }$, and evaluated peak-to-peak envelope (blue) for one fundamental period: (a) simulation, with details, and (b) experimental results.

(a)

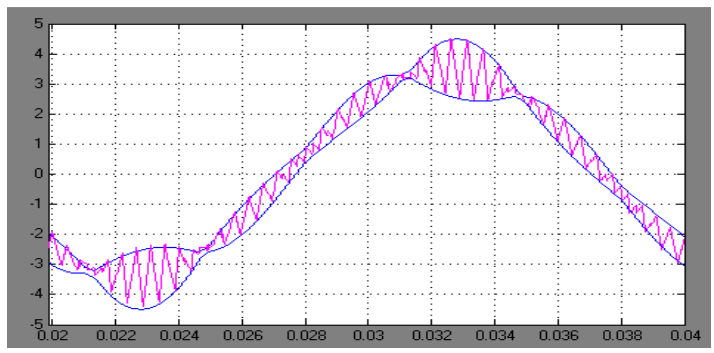

(b)

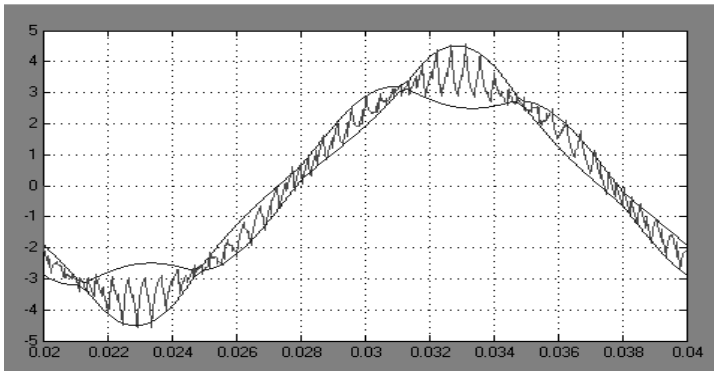

Fig. 2.25. Instantaneous output current (pink) with calculated current envelopes (blue traces) for $m=1 / \sqrt{ } 3=m_{\max }$ : (a) simulated, (b) experimental results. 


\subsection{References}

[1] A.M. Hava, R. J. Kerkman, T.A. Lipo, "Carrier-based PWM-VSI overmodulation strategies: analysis, comparison, and design," IEEE Trans. On Power Electronics, vol. 13, no. 4, pp. 674-689, July 1998.

[2] D. Zhao, V.S.S. Pavan Kumar Hari, G. Narayanan, R. Ayyanar, "Spacevector hybrid pulsewidth modulation techniques for reduced harmonic distortion and switching loss," IEEE Trans. On Power Electronics, vol. 25, no. 3, pp. 760-774, March 2010.

[3] D. Casadei, M. Mengoni, G. Serra, A. Tani, L. Zarri, "A new carrierbased PWM strategy with minimum output current ripple for five-phase inverters," In Proc. of the $14^{\text {th }}$ European Conference on Power Electronics and Applications (EPE), Birmingham UK, 30. Aug. 2011- 1. Sept. 2011, pp. 1-10.

[4] X. Mao, R. Ayyanar, H.K. Krishnamurthy, "Optimal variable switching frequency scheme for reducing switching loss in single-phase inverters based on time-domain ripple analysis," IEEE Trans. On Power Electronics, vol. 24, no. 4, pp. 991-1001, April 2009.

[5] G.D. Holmes, T.A. Lipo (2003), "Pulse width modulation for power converters - principles and practice," IEEE Press Series on Power Engineering, John Wiley and Sons, Piscataway, NJ, USA.

[6] J. W. Kolar, H. Ertl, F.C. Zach, "Influence of the modulation method on the conduction and switching losses of a PWM converter system," IEEE Trans. On Industry applications, vol. 27, no. 6, pp. 1063-1075, November/December 1991.

[7] J.W. Kolar, H. Ertl, F.C. Zach, “ Minimizing the current harmonics RMS value of three-phase PWM converter systems by optimal and suboptimal transition between continuous and discontinuous modulation," In Proc. of $22^{\text {nd }}$ Annual IEEE Power Electronics Specialists Conference (PESC '91), Cambridge MA, 24-27 Jun 1991, pp. 372-381.

[8] E. Levi, D. Dujic, M. Jones, G. Grandi, “Analytical determination of DCbus utilization limits in multi-phase VSI supplied AC drives," IEEE Trans. on Energy Convers., vol. 23, no. 2, pp. 433-443, June 2008. 
[9] D. Casadei, G. Serra, A. Tani, L. Zarri, "Theoretical and experimental analysis for the RMS current ripple minimization in induction motor drives controlled by SVM technique," IEEE Transactions on Industrial Electronics, vol. 51, no. 5, pp. 1056-1065, Oct. 2004.

[10] G. Narayanan, D. Zhao, H.K. Krishnamurthy, R. Ayyanar, V.T. Ranganathan, "Space vector based hybrid PWM techniques for reduced current ripple," IEEE Trans. On Industrial Electronics, vol. 55, no. 4, pp. 1614-1627, April 2008.

[11] T.B. Reddy, J. Amarnath, D. Subbarayudu, "New hybrid SVPWM methods for direct torque controlled induction motor drive for reduced current ripple," In Proc. of International Conference on Power Electronics, Drives and Energy Systems (PEDES '06), New Delhi IN, 1215 Dec. 2006, pp. 1-6.

[12] A. Murnandityo, P.A. Dahono, "Analysis of output current ripple of three-phase PWM inverter under discontinuous modulation techniques," Industrial Electronic Seminar, 2010.

[13] D. Dujic, M. Jones, E. Levi, "Analysis of output current ripple rms in multiphase drives using space vector approach," IEEE Trans. On Power Electronics, vol. 24, no. 8, pp. 1926-1938, August 2009.

[14] M. Jones, D. Dujic, E. Levi, J. Prieto, F. Barrero, "Switching ripple characteristics of space vector PWM schemes for five-phase two-level voltage source inverters-Part2: Current ripple," IEEE Trans. On Industrial Electronics, vol. 58, no. 7, pp. 2799-2808, July 2011.

[15] P.A. Dahono, Deni, E.G. Supriatna, "Output current-ripple analysis of five-phase PWM inverters," IEEE Trans. On Industry Applications, vol. 45, no. 6, pp. 2022-2029, November/December 2009.

[16] D. Dujic, M. Jones, E. Levi, "Analysis of output current-ripple RMS in multiphase drives using polygon approach," IEEE Trans. Power Electron. vol. 25, no. 7, pp. 1838-1849, July 2010.

[17] Jiang, D., Wang, F, "Current-ripple prediction for three-phase PWM converters," IEEE Trans. on Ind. Applications, vol. 50, no. 1, pp. 531538, Jan/Feb 2014. 
[18] D. Jiang, F. Wang, "Variable switching frequency PWM for three-phase converters based on current ripple prediction," IEEE Trans. on Power Electronics, vol. 28, no. 11, pp. 4951-4961, Nov. 2013.

[19] G. Grandi, J. Loncarski, R. Seebacher, "Effects of current ripple on dead-time analysis of three-phase inverters, " In Proc. of IEEE Energy Conference (ENERGYCON), Florence IT, 9-12 Sept. 2012, pp. 207212.

[20] G. Grandi and J. Loncarski, "Analysis of dead-time effects in multi-phase voltage sourse inverters," In Proc. of IET Power Electronics, Machines and Drives (PEMD), Bristol UK, 27-29 March 2012, CD-ROM paper 0223 .

\subsection{Authored papers}

- G. Grandi, J. Loncarski, "Evaluation of current ripple amplitude in threephase PWM voltage source inverters," In Proc. of $8^{\text {th }}$ IEEE Intl. Conference on Compatibility and Power Electronics, CPE, Ljubljana SLO, 5-7 June 2013. DOI: $10.1109 /$ CPE.2013.6601146 


\section{Analysis of the output current ripple in multiphase VSIs}

\subsection{Introduction}

The area of multiphase systems has experienced a substantial growth since the beginning of this century. All multiphase motor drives are supplied by the multiphase voltage source inverters. An example of the multiphase inverter supplying a RL load is represented in Fig. 3.1. Multiphase power systems have many advantages over the traditional three-phase counterparts. There are few important reasons for the emerged interest in multiphase systems. The efficiency is higher than in a three-phase machine due to the fact that stator excitation in a multiphase machine produces a field with a lower spaceharmonic content. Another advantage is that multiphase machines have a greater fault tolerance than their three-phase counterparts. This is especially true in means when open-circuit occurs. Furthermore, multiphase machines are less sensitive than their three-phase counterparts to time-harmonic components in the excitation waveform, whose components produce pulsating torques at even multiples of the fundamental excitation frequency. Another important

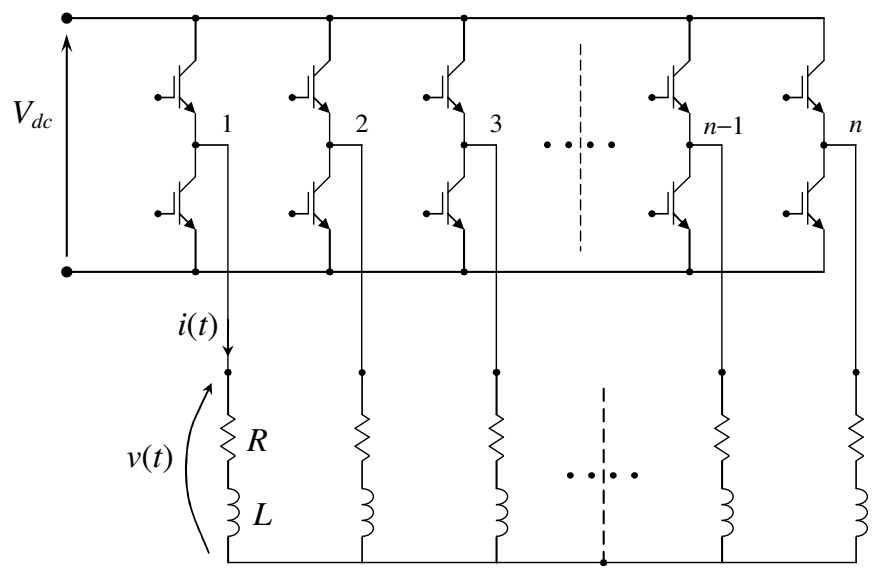

Fig. 3.1. Multiphase inverter supplying a $R L$ load 
reason for employing multiphase motor variable-speed drives is in the high power drives, because of the possibility of reducing the required rating of power electronic components for the given motor output power, when compared to a three-phase motor drive. Utilization of multiphase motor drives also enables improvement in the noise characteristics [1].

Additionally, besides that multiphase motor drives have the ability to reduce the amplitude and increase the frequency of torque pulsations, to reduce the rotor harmonic current losses and to lower the dc link current harmonics multiphase systems, they improve the reliability owing to their redundant structure [2]-[7]. The use of multiphase inverters together with multiphase ac machines has been recognized as a viable approach to obtain high power ratings with current limited devices.

\subsubsection{Modulation techniques}

In CB-PWM schemes the $n$ - phase reference voltages are compared with triangle carrier waveform. The frequency of these carriers defines the switching frequency, and their amplitude is the half of the dc-link voltage, eventually in per units. The range of the modulation index defined as $m=V^{*} / V_{d c}$ in this case is $0<m<0.5$. By centering the modulation signals, and optimal modulation from the point of view of output current ripple RMS minimization and better harmonic spectrum is obtained. The common-mode voltage defined

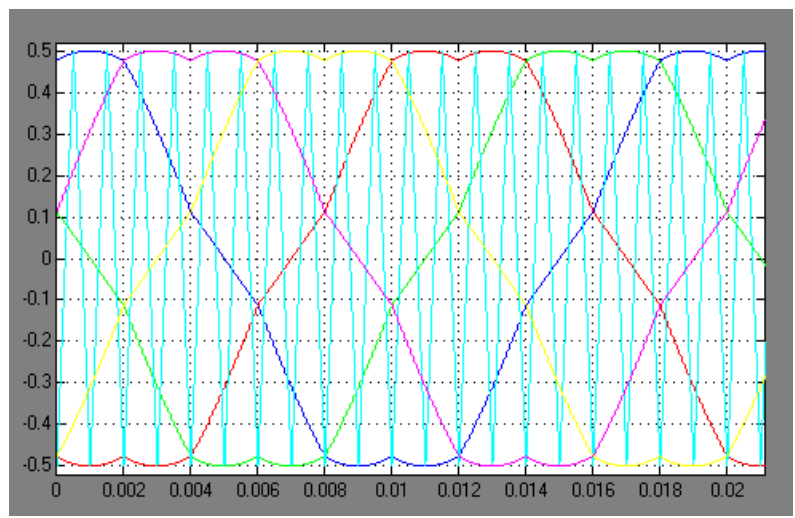

Fig. 3.2. Carrier based modulation of five-phase inverter with centered reference voltages, $m=$ $m_{\max }=0.526$ 
by (2.1), considering $k=\mathrm{a}, \mathrm{b}, \mathrm{c}, \ldots \mathrm{n}$, is added to the $n$ - phase references, and new references able to obtain centered carrier-based modulation are presented in Fig. 3.2, for the example of the five-phase inverter. Modulation limit is in this case defined according to the generalized expression given in [6] for $n$ phases, $m_{\max }=[2 \cos (\pi / 2 n)]^{-1}$, i.e. for five-phase $m_{\max }=0.526$, and for sevenphase inverter $m_{\max }=0.513$.

The behavior of multiphase systems can be represented by the multiple space vector theory, as a natural extension of the traditional three-phase space vector transformation, leading to an elegant and effective vectorial approach in multiple $\alpha-\beta$ planes [7]. In particular, the space vectors can be usefully adopted for the modulation of multiphase inverters. The space vector modulation for five-phase voltage source VSIs has been developed in [8]-[11]. Analysis of seven-phase inverters and their modulation is given in [12]-[16]. The principle of SV-PWM in multiphase inverters is summarized in Fig. 3.3 for five-phase and Fig. 3.4 for seven-phase inverters, representing the output voltage space vectors corresponding to all possible switch configurations.

In general, for any number of phases, it has been proven that the SV-PWM provides the same switching pattern such as the CB-PWM when a "min/max centered" common-mode voltage is injected into the modulating signals. By equally sharing the application time of the null voltage vector between the two null switch configurations, the centered switching pattern is realized, and nearly-optimal modulation able to minimize the RMS of current ripple is obtained [14], [17].
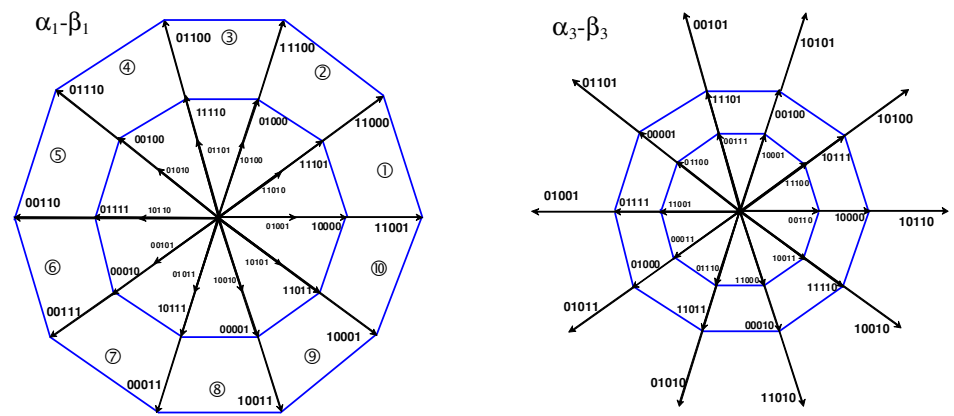

Fig. 3.3. Space vector diagrams of five-phase inverter output voltage in the planes $\alpha_{1}-\beta_{1}$ and $\alpha_{3}-\beta_{3}$. 

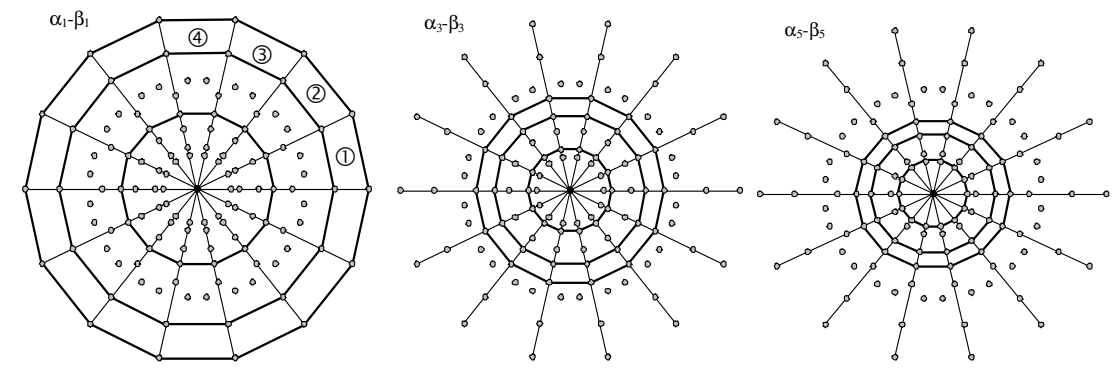

Fig. 3.4. Space vector diagrams of seven-phase inverter output voltage in the planes $\alpha_{1}-\beta_{1}$, $\alpha_{3}-\beta_{3}$, and $\alpha_{5}-\beta_{5}$.

\subsection{Extension of the peak-to-peak output current ripple analysis to five- phase VSIs}

\subsubsection{State of the art}

Also in the case of the multiphase inverters, the output current ripple is studied in the terms of the overall effect, i.e. its RMS value, rather than the peak-to-peak amplitude. Recent studies about RMS of the output current ripple in multiphase motor drives are given in [18]-[21], considering a five-phase system. In [18] the optimal value of the common-mode voltage injection in CBPWM has been analytically determined to minimize the RMS current ripple in each switching period. Furthermore, it is shown that the strategy called SVPWM, corresponding to centered and symmetric modulation, has a nearlyoptimal behavior in term of the current ripple RMS. In [19] is shown that output current ripple RMS cannot be minimized by injection of fifth harmonic and its odd multiples, but it is also pointed out that, from the practical point of view, differences in current ripple RMS are relatively small considering sinusoidal PWM and SV-PWM. In [20] two SV-PWM techniques are compared (four large vs. two large and two middle vectors) in terms of THD of the current and voltage with established correlations between the flux HDF and the current THD, and squared RMS current ripple. In [21] an attempt to evaluate the output RMS current ripple has been reported, on the basis of polygon load connection and phase variables in the original domain. However, only a 
single (adjacent) polygon connection has been used, and the calculated output current ripple RMS does not represent the total output current ripple [22].

In addition to the RMS analysis, the benefit of the peak-to-peak current ripple evaluation is recognized in [23], where the ripple amplitude is investigated for three-phase PWM inverters. This analysis has been extended to the multiphase case [24].

\subsubsection{Calculation of the peak-to-peak output current ripple}

The analysis of five-phase inverters by the space vector transformation leads to better understanding and more simple calculation of voltage levels and corresponding application times. In the following analysis is considered the centered PWM, as described in Sect. 3.1.1. The instantaneous current ripple is introduced for a generic balanced five-phase $R-L-E M F$ load (RLE). The analysis can be extended to five-phase ac motor drives as well, but the circuit model of the specific ac motor must be carefully considered to evaluate the cases in which it can be reduced to the basic RLE equivalent circuit [19, 22].

Multiple space vectors are introduced to represent voltage and current phase quantities in multiphase systems [7]. For the five-phase system $\left\{x_{1}, x_{2}, x_{3}, x_{4}\right.$, $\left.x_{5}\right\}$, the two space vectors $x_{1}$ and $x_{3}$ lie in the two planes $\alpha_{1}-\beta_{1}$ and $\alpha_{3}-\beta_{3}$, respectively, and are expressed as

$$
\left\{\begin{array}{l}
\boldsymbol{x}_{1}=\frac{2}{5}\left[x_{1}+x_{2} \boldsymbol{\alpha}+x_{3} \boldsymbol{\alpha}^{2}+x_{4} \boldsymbol{\alpha}^{3}+x_{5} \boldsymbol{\alpha}^{4}\right] \\
\boldsymbol{x}_{3}=\frac{2}{5}\left[x_{1}+x_{2} \boldsymbol{\alpha}^{3}+x_{3} \boldsymbol{\alpha}+x_{4} \boldsymbol{\alpha}^{4}+x_{5} \boldsymbol{\alpha}^{2}\right] \\
x_{0}=\frac{1}{5}\left[x_{1}+x_{2}+x_{3}+x_{4}+x_{5}\right]
\end{array}\right.
$$

being $\boldsymbol{\alpha}=\exp (j 2 \pi / 5)$ and the $x_{0}$ the zero-sequence component, always null in case of balanced systems.

The inverse transformation of (3.1) is

$$
x_{k}=x_{0}+\boldsymbol{x}_{1} \cdot \boldsymbol{\alpha}^{(k-1)}+\boldsymbol{x}_{3} \cdot \boldsymbol{\alpha}^{3(k-1)}, k=1,2, \ldots, 5 .
$$


With reference to a five-phase VSI supplied by the dc voltage $V_{d c}$, the output space voltage vectors can be written as function of the five switching leg states $S_{k}=[0,1]$ as

$$
\left\{\begin{array}{l}
v_{1}=\frac{2}{5} V_{d c}\left[S_{1}+S_{2} \boldsymbol{\alpha}+S_{3} \boldsymbol{\alpha}^{2}+S_{4} \boldsymbol{\alpha}^{3}+S_{5} \boldsymbol{\alpha}^{4}\right] \\
v_{3}=\frac{2}{5} V_{d c}\left[S_{1}+S_{2} \boldsymbol{\alpha}^{3}+S_{3} \boldsymbol{\alpha}+S_{4} \boldsymbol{\alpha}^{4}+S_{5} \boldsymbol{\alpha}^{2}\right]
\end{array} .\right.
$$

The space vector diagrams representing all possible switch configurations in planes $\alpha_{1}-\beta_{1}$ and $\alpha_{3}-\beta_{3}$ are given in Fig. 3.3.

The SV-PWM of five-phase inverters is based on the determination of application times of active and null inverter voltage vectors $\boldsymbol{v}_{1}$ and $\boldsymbol{v}_{3}$ in every switching period $T_{s}$. In the case of symmetrical SV-PWM, the sequence is determined in $T_{s} / 2$ and symmetrically repeated in the next half switching period. As result of this modulation, the average of the inverter output voltage $\bar{v}\left(T_{s}\right)$ corresponds to the reference voltage $v^{*}$, for each phase.

In the case of sinusoidal balanced output voltages supplying a balanced load, the zero-sequence component is null. Introducing the modulation index $m$ $=V^{*} / V_{d c}$, the reference space voltage vectors become

$$
\left\{\begin{array}{l}
v_{1}^{*}=v^{*}=m V_{d c} e^{j \vartheta} \\
v_{3}^{*}=0 .
\end{array}\right.
$$

In this case, SV modulation is quarter-wave symmetric, and it can be analyzed in the range $[0, \pi / 2]$ of the phase angle $\vartheta=\omega t$. With reference to Fig. 3.5 , the sectors (1) and (2) are considered for $0 \leq \vartheta \leq \pi / 5$ and $\pi / 5 \leq \vartheta \leq 2 \pi / 5$, respectively, and the half of sector (3) is considered for $2 \pi / 5 \leq \vartheta \leq \pi / 2$.

For sector (1) the application times of the switch configurations involved in the modulation sequence from 00000 to 11111 in the half period $T_{s} / 2$ can be determined as [8]

$$
\begin{array}{ll}
t_{1}=m T_{s} K_{1} \sin (\pi / 5-\vartheta), & \{10000\} \\
t_{2}=m T_{s} K_{3} \sin \vartheta, & \{11000\} \\
t_{3}=m T_{s} K_{3} \sin (\pi / 5-\vartheta), & \{11001\}
\end{array}
$$




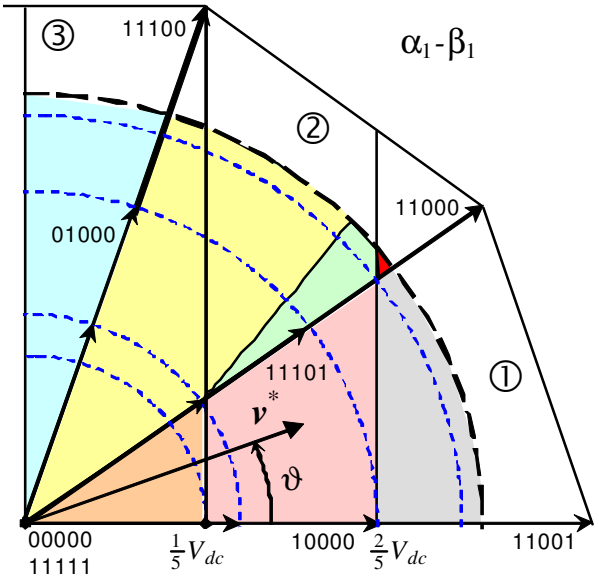

Fig. 3.5. Space vector diagram of inverter output voltage on plane $\alpha_{1}-\beta_{1}$ in the range $\vartheta=\left[0,90^{\circ}\right]$. Outer dashed circle is the modulation limit, $m_{\max } \approx 0.526$. Colored areas represent different equations to determine the current ripple.

$$
\begin{aligned}
& t_{4}=m T_{s} K_{1} \sin \vartheta,\{11101\} \\
& t_{0}=\frac{T_{s}}{2}-\left(t_{1}+t_{2}+t_{3}+t_{4}\right)= \\
&=\frac{T_{s}}{2}\left[1-m\left(1+\cos \frac{\pi}{5}\right) \cos \vartheta-m K_{1} \sin \vartheta\right],\{00000\} \\
&\{11111\}
\end{aligned}
$$

being

$$
\left\{\begin{array}{l}
K_{1}=\sin \frac{\pi}{5} \cong 0.588 \\
K_{3}=\sin \frac{3 \pi}{5} \cong 0.951 .
\end{array}\right.
$$

Equations (3.5) - (3.9) can be extended to any sector $k$ by replacing the phase angle $\vartheta$ by $\vartheta-(k-1) \pi / 5, k=1,2, \ldots, 10$.

Due to the symmetry among all phases in the considered case of sinusoidal balanced currents, only the first phase is examined in the following analysis. In terms of multiple space vectors, the phase variables are given by (3.2). For the 
first phase, it results in the projection of the two space vectors on the real axes. In particular, introducing (3.4) in (3.2), the average output voltage of the first phase is given by

$$
\bar{v}\left(T_{s}\right)=v^{*}=\Re e\left\{\boldsymbol{v}_{1}^{*}\right\}+\Re e\left\{\boldsymbol{v}_{3}^{*}\right\}=m V_{d c} \cos \vartheta .
$$

By introducing (3.11) in the expression for alternating voltage (2.6), and calculating $v(t)$ by (3.2) and (3.3), the alternating component of inverter output voltage of the first phase can be written as

$$
\tilde{v}(t)=\left[S_{1}-\frac{1}{5}\left(S_{1}+S_{2}+S_{3}+S_{4}+S_{5}\right)\right] V_{d c}-m V_{d c} \cos \vartheta .
$$

In order to evaluate the current ripple in the whole phase angle range $0<$ $\vartheta<\pi / 2$, the three main cases corresponding to the three sectors depicted in Fig. 3.5 should be considered. Additional sub-cases, also determined by the value of modulation index are identified in Fig. 3.5 with different colors.

The current ripple is depicted in a separate diagram for each sector, from Fig. 3.6 to Fig. 3.8. In general, the ripple shows 2 peaks in the switching period (two positive and the symmetric negative). For all cases, one peak always bigger than the other in the considered sector, for any specific range of $m \cos \vartheta$, due to current slopes and application times. The only exception is in the second sector (2), where either one or the other peak is bigger depending on the values of $m$ and $\vartheta$.

\section{1) Evaluation in the first sector}

Considering sector (1) $0 \leq \vartheta \leq \pi / 5$, three different sub-cases can be distinguished: $0 \leq m \cos \vartheta \leq 1 / 5,1 / 5 \leq m \cos \vartheta \leq 2 / 5$, and $2 / 5 \leq m \cos \vartheta \leq m_{\max } \cos \vartheta<$ 3/5. All this sub-cases are represented in Fig. 3.6.

The sub-case $0 \leq m \cos \vartheta \leq 1 / 5$ (orange area in Fig. 3.5) is considered in diagram 1 of Fig. 3.6, where the current ripple $\tilde{i}$ and its peak-to-peak value $\tilde{i}_{p p}$ are depicted, together with the instantaneous output voltage $v(t)$. In this case, according to Fig. 3.6, $\tilde{i}_{p p}$ can be evaluated by the expressions for the instantaneous current ripple and its peak-to-peak value, (2.10), (2.11), and (3.12) considering switch configurations 11111 or 00000 with the corresponding application interval $t_{0}$, leading to 


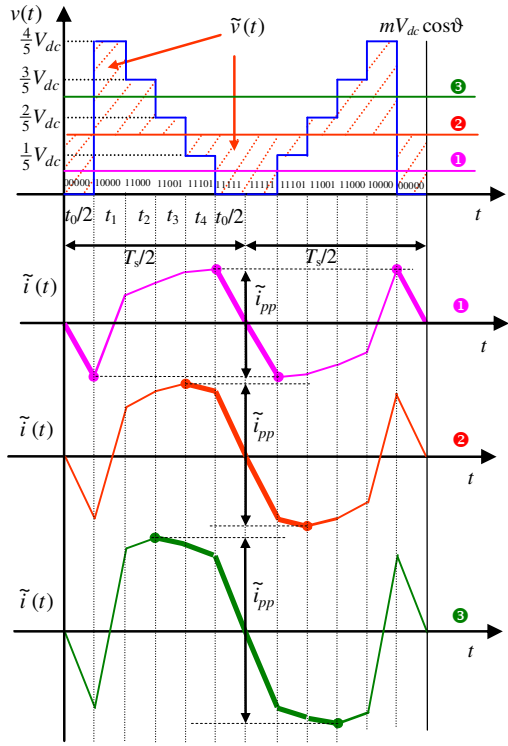

Fig. 3.6. Output voltage and current ripple in one switching period for sector (1), $0 \leq \vartheta \leq \pi / 5$

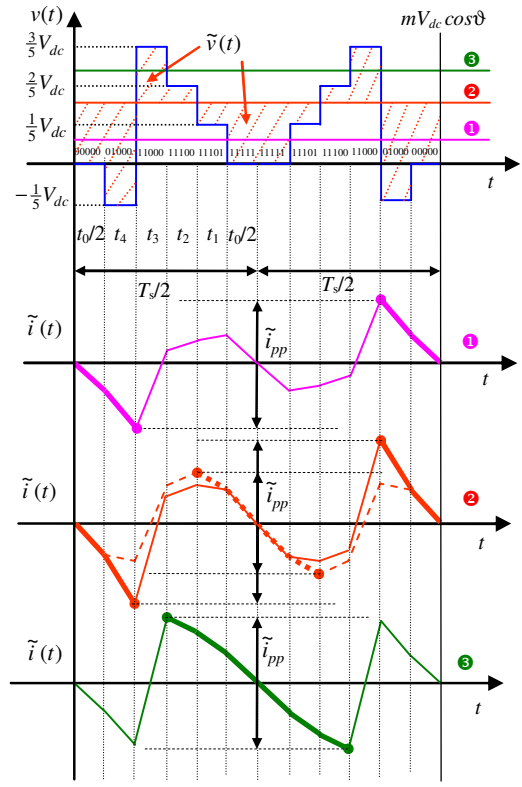

Fig. 3.7. Output voltage and current ripple in one switching period for sector (2), $\pi / 5 \leq \vartheta \leq$ $2 \pi / 5$.

$$
\tilde{i}_{p p}=\frac{1}{L}\left\{m V_{d c} \cos \vartheta t_{0}\right\} .
$$

Peak-to-peak current ripple can also be expressed as

$$
\tilde{i}_{p p}=\frac{V_{d c} T_{s}}{2 L} r(m, \vartheta),
$$

being $r(m, \vartheta)$ the normalized peak-to-peak current ripple amplitude.

Introducing the duty-cycle $\delta_{k}=t_{k} / T_{s} / 2$, the expression for the normalized peak-to-peak current ripple in this case is

$$
r(m, \vartheta)=m \cos \vartheta \delta_{0}
$$

The sub-case $1 / 5 \leq m \cos \vartheta \leq 2 / 5$ (pink area in Fig. 3.5) is depicted in diagram 2 of Fig. 3.6. In this case $\widetilde{i}_{p p}$ can be evaluated considering the switch 
configurations 11111 and 11101, with corresponding application intervals $t_{0} / 2$ and $t_{4}$, leading to

$$
\tilde{i}_{p p}=\frac{2}{L}\left\{m V_{d c} \cos \vartheta \frac{t_{0}}{2}+\left(m V_{d c} \cos \vartheta-\frac{V_{d c}}{5}\right) t_{4}\right\} .
$$

Normalizing by (3.14) and introducing the duty-cycles, the normalized current ripple becomes

$$
r(m, \vartheta)=m \cos \vartheta \delta_{0}+2\left(m \cos \vartheta-\frac{1}{5}\right) \boldsymbol{\delta}_{4} .
$$

The sub-case $2 / 5 \leq m \cos \vartheta \leq m_{\max } \cos \vartheta$ (gray area in Fig. 3.5) is depicted in diagram 3 of Fig. 3.6. In this case $\tilde{i}_{p p}$ can be evaluated considering the switch configurations 11111, 11101, and 11001, with the corresponding application intervals $t_{0} / 2, t_{4}$, and $t_{3}$, leading to

$$
\begin{aligned}
\tilde{i}_{p p}=\frac{2}{L}\left\{m V_{d c} \cos \vartheta\right. & \frac{t_{0}}{2} \\
+ & \left(m V_{d c} \cos \vartheta-\frac{V_{d c}}{5}\right) t_{4}+ \\
& \left.+\left(m V_{d c} \cos \vartheta-\frac{2 V_{d c}}{5}\right) t_{3}\right\} .
\end{aligned}
$$

The corresponding normalized current ripple is

$$
r(m, \vartheta)=m \cos \vartheta \delta_{0}+2\left(m \cos \vartheta-\frac{1}{5}\right) \delta_{4}+2\left(m \cos \vartheta-\frac{2}{5}\right) \delta_{3} .
$$

2) Evaluation in the second sector

Considering sector (2), $\pi / 5 \leq \vartheta \leq 2 \pi / 5$, three different sub-cases can distinguished: $0 \leq m \cos \vartheta \leq 1 / 5,1 / 5 \leq m \cos \vartheta \leq 2 / 5$, and $2 / 5 \leq m \cos \vartheta \leq m_{\max } \cos \vartheta<$ 3/5. All these sub-cases are represented in Fig. 3.7.

The sub-case $0 \leq m \cos \vartheta \leq 1 / 5$ (yellow area in Fig. 3.5) is depicted in diagram 10 of Fig. 3.7. According to this figure, $\tilde{i}_{p p}$ can be evaluated by (2.10), (2.11) and (3.12) considering the switch configurations 00000 and 01000 with corresponding application intervals $t_{0} / 2$ and $t_{4}$, leading to 


$$
\tilde{i}_{p p}=\frac{2}{L}\left\{m V_{d c} \cos \vartheta \frac{t_{0}}{2}+\left(m V_{d c} \cos \vartheta+\frac{1}{5} V_{d c}\right) t_{4}\right\}
$$

Normalizing by (3.14) and introducing the duty-cycles, the normalized current ripple becomes

$$
r(m, \vartheta)=m \cos \vartheta \delta_{0}+2\left(m \cos \vartheta+\frac{1}{5}\right) \delta_{4} .
$$

The sub-case $1 / 5 \leq m \cos \vartheta \leq 2 / 5$ is depicted in diagram 2 of Fig. 3.7. There are two possible situations for evaluating $\tilde{i}_{p p}$, corresponding to the yellowgreen areas of sector (2) in Fig. 3.5:

- in the first situation, yellow area, (solid orange line in Fig. 3.7), $\tilde{i}_{p p}$ can be determined as in previous sub-case by considering switch configurations 00000 and 01000 with corresponding application intervals $t_{0} / 2$ and $t_{4}$, leading to (3.20) and (3.21);

- in the second situation, green area (dashed orange line in Fig. 3.7), $\tilde{i}_{p p}$ can be determined by considering the switch configurations 11111 and 11101 with the corresponding application intervals $t_{0} / 2$ and $t_{1}$, leading to

$$
\tilde{i}_{p p}=\frac{2}{L}\left\{m V_{d c} \cos \vartheta \frac{t_{0}}{2}+\left(m V_{d c} \cos \vartheta-\frac{1}{5} V_{d c}\right) t_{1}\right\} \text {. }
$$

The normalized current ripple is

$$
r(m, \vartheta)=m \cos \vartheta \delta_{0}+2\left(m \cos \vartheta-\frac{1}{5}\right) \delta_{1} .
$$

The last sub-case $2 / 5 \leq m \cos \vartheta \leq m_{\max } \cos \vartheta$ (red area in Fig. 3.5) is depicted in diagram 3 of Fig. 3.7. According to this figure, $\tilde{i}_{p p}$ can be evaluated considering the switch configurations 11111, 11101, and 11100 with the corresponding application intervals $t_{0} / 2, t_{1}$, and $t_{2}$, leading to

$$
\begin{aligned}
\tilde{i}_{p p}=\frac{2}{L}\left\{m V_{d c} \cos \vartheta\right. & \frac{t_{0}}{2}+\left(m V_{d c} \cos \vartheta-\frac{V_{d c}}{5}\right) t_{1}+ \\
& \left.+\left(m V_{d c} \cos \vartheta-\frac{2 V_{d c}}{5}\right) t_{2}\right\} .
\end{aligned}
$$


The normalized current ripple in this case is

$$
r(m, \vartheta)=m \cos \vartheta \delta_{0}+2\left(m \cos \vartheta-\frac{1}{5}\right) \delta_{1}+2\left(m \cos \vartheta-\frac{2}{5}\right) \delta_{2} .
$$

\section{3) Evaluation in the third sector (half)}

With reference to the half of the sector (3), $2 \pi / 5 \leq \vartheta \leq \pi / 2$ (blue area in Fig. $3.5)$, there are not sub-cases, and the only occurrence is $0 \leq m \cos \vartheta \leq m_{\max }$ $\cos \vartheta<1 / 5$, as depicted in Fig. 3.8. In this case, $\tilde{i}_{p p}$ can be evaluated by (2.10), (2.11) and (3.12), considering the switch configurations 00000, 01000, and 01100 with the corresponding application intervals $t_{0} / 2, t_{1}$ and $t_{2}$, leading to

$$
\begin{aligned}
\tilde{i}_{p p}=\frac{2}{L}\left\{m V_{d c} \cos \vartheta \frac{t_{0}}{2}\right. & +\left(m V_{d c} \cos \vartheta+\frac{1}{5} V_{d c}\right) t_{1}+ \\
& \left.+\left(m V_{d c} \cos \vartheta+\frac{2}{5} V_{d c}\right) t_{2}\right\} .
\end{aligned}
$$

Normalized current ripple is

$$
r(m, \vartheta)=m \cos \vartheta \delta_{0}+2\left(m \cos \vartheta+\frac{1}{5}\right) \delta_{1}+2\left(m \cos \vartheta+\frac{2}{5}\right) \delta_{2}
$$

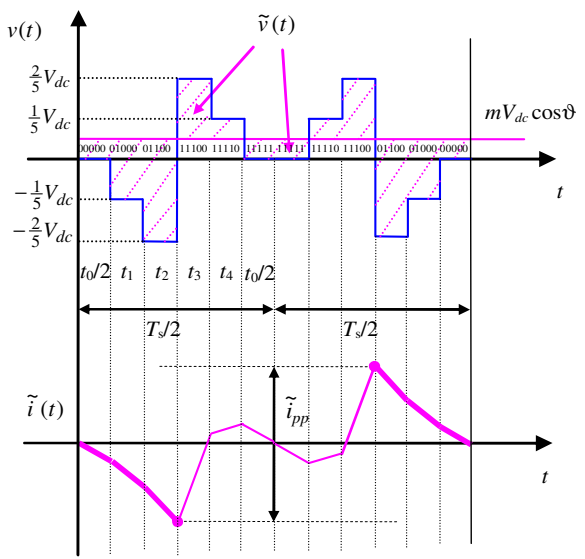

Fig. 3.8. Output voltage and current ripple in one switching period for sector (3), $2 \pi / 5 \leq \vartheta \leq \pi / 2$. 


\subsubsection{Peak-to-peak current ripple diagrams}

In order to show the behavior of the peak-to-peak current ripple amplitude in the fundamental period for all the possible cases, in Figs. 3.9 and 3.10 is represented the normalized function $r(m, \vartheta)$ defined by (3.14). Fig. 3.9 shows $r(\vartheta)$ for $m=0.2,0.247,0.4$, and $0.494(=2 \times 0.247)$, corresponding to the dashed circles in Fig. 3.5. The three ranges of $\vartheta$ correspond to the three sectors from (1) to (3). The further sub-region in sector (2) (green- and red-colored areas in Fig. 3.5) can be distinguished for $m=0.4$.

Fig. 3.10 shows the colored map of $r(m, \vartheta)$ for the first quadrant within the modulation limits. It can be noted that ripple amplitude is obviously zero for $m=0$, since the null configurations are the only applied, increasing almost proportionally with $m$ in the neighborhoods of $m=0$. Phase angle with minimum ripple can be identified, that is $\vartheta \approx 40^{\circ} \div 45^{\circ}$, and a phase angle with maximum ripple, that is $\vartheta=90^{\circ}$, with ripple amplitude proportional to modulation index: $r\left(m, 90^{\circ}\right)=2 / 5\left[K_{1}+K_{3}\right] m \approx 0.616 m$, resulting from (3.27). This aspect is further developed in the following sub-section.

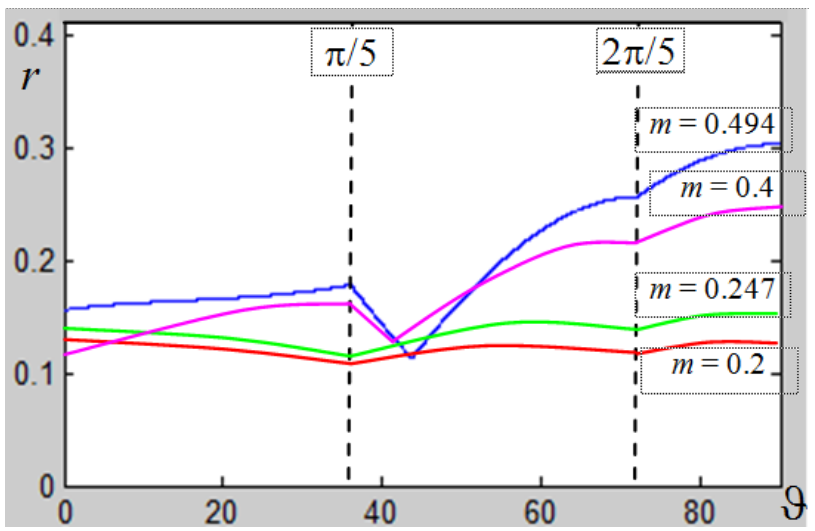

Fig. 3.9. Normalized peak-to-peak current ripple amplitude $r(m, \vartheta)$ for different modulation indexes in the phase angle range $\vartheta=[0, \pi / 2]$. 


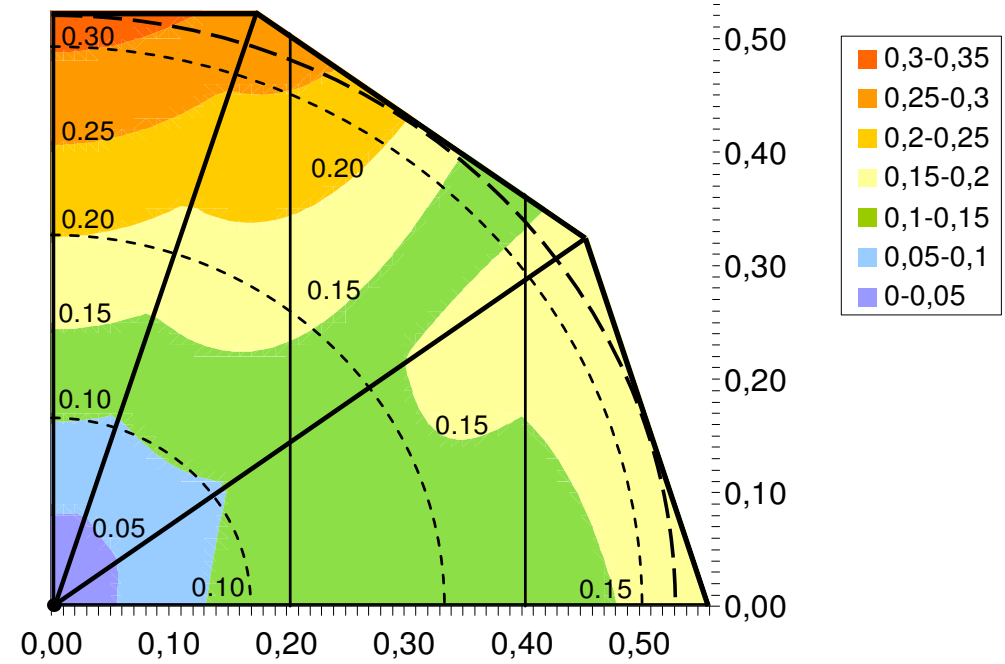

Fig. 3.10. Map of the normalized peak-to-peak current ripple amplitude $r(m, \vartheta)$

\subsubsection{Maximum of the current ripple}

In order to estimate current ripple amplitude in the whole fundamental period, the maximum of the current ripple can be evaluated in the phase angle range $\left[0,90^{\circ}\right]$. For this purpose, two relevant angles can be observed in Figs. 3.9 and 3.10: a local maximum for $\vartheta=0^{\circ}$, and further local maximum for $\vartheta=90^{\circ}$. In particular, to determine these two local maxima, it can be set $\vartheta=0$ in (3.15), and $\vartheta=90^{\circ}$ in (3.27), introducing the application times given by (3.5) - (3.9). The maximum value of normalized peak-to-peak current ripple amplitude as a function of the modulation index becomes

$$
r^{\max }(m)=\max \left\{m\left[1-2 m K_{1}^{2}-2 m K_{1} K_{3}\right], \frac{2}{5}\left[K_{1}+K_{3}\right] m\right\} .
$$

The intersection between the two local maxima gives the border value of modulation index

$$
m\left[1-2 m K_{1}^{2}-2 m K_{1} K_{3}\right]=\frac{2}{5}\left[K_{1}+K_{3}\right] m,
$$




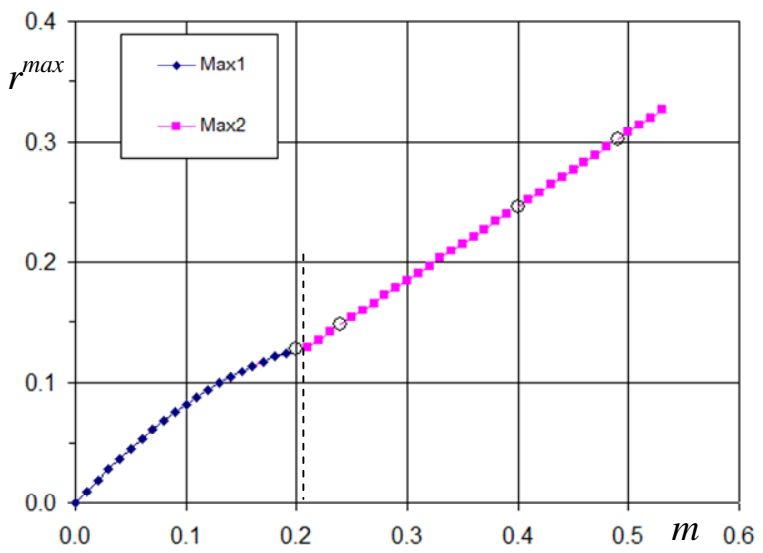

Fig. 3.11. Maximum of the normalized peak-to-peak current ripple amplitude as function of modulation index.

leading to $m \approx 0.212$. Finally, combining (3.28) and (3.29), the maximum of the normalized current ripple is

$$
r^{\max }(m)=\left\{\begin{array}{l}
m\left[1-2 m K_{1}{ }^{2}-2 m K_{1} K_{3}\right] \text { for } m \leq 0.212, \\
\frac{2}{5}\left[K_{1}+K_{3}\right] m \quad \text { for } m \geq 0.212 .
\end{array}\right.
$$

The composition of the two local maxima is given in Fig. 3.11, leading to the global maximum. The four white dots represent the specific points for $m=0.2$, $0.247,0.4$, and 0.494 , displayed in Fig. 3.9. It can be noted that maximum function is almost linear with the modulation index, strictly for $m>0.212$. Then, on the basis of (3.30) and (3.14), a simplified expression for maximum of peakto-peak current ripple amplitude can be obtained

$$
\tilde{i}_{p p}{ }^{\max }=\frac{V_{d c} T_{s}}{5 L} m\left[K_{1}+K_{3}\right] \cong \frac{V_{d c} T_{s}}{3.25 L} m
$$




\subsection{Numerical and experimental results}

In order to verify the theoretical developments proposed in previous sections, numerical simulations and corresponding experimental tests are carried out. Circuit simulations are preformed by Sim-PowerSystem (Matlab) considering a five-phase VSI with ideal switches supplying a balanced RL load, having $R=22 \Omega$ and $L=8 \mathrm{mH}$. In all simulations the fundamental frequency $f$ is set to $50 \mathrm{~Hz}$, the switching frequency $1 / T_{s}$ is $2 \mathrm{kHz}$, and the dc voltage supply $V_{d c}$ is $100 \mathrm{~V}$. A centered symmetrical carrier-based PWM technique is considered in both simulations and experiments, being equivalent to the centered space vector modulation but easier for the implementation.

The experimental setup with custom-built two-level multiphase inverter is shown in Fig. 3.12. Power switches are Infineon FS50R12KE3 IGBT pack. For the real-time implementation of the algorithm, dSpace ds1006 hardware has been employed. The dc-bus voltage $\left(V_{d c}\right)$ is set to $100 \mathrm{~V}$ by Sorensen SGI $600 / 25 \mathrm{dc}$ supply. The inverter switching frequency $\left(1 / T_{s}\right)$ was set to $2 \mathrm{kHz}$, with inverter's dead-time of $6 \mu$ s (not compensated) implemented in the hardware. The load parameters are: $R=22 \Omega$ and $L=8 \mathrm{mH}$.

The instantaneous current ripple $\tilde{i}$ in both simulations and experiments is calculated as the difference between the instantaneous current and its fundamental component, i.e.

$$
\tilde{i}(t)=i(t)-I_{\text {fund }}(t) .
$$

In the experimental results has been noticed that the ripple was with high $3^{\text {rd }}$ harmonic which was the consequence of the inverter dead-time (5-th harmonic is missing and other odd harmonics were negligible [25]). In order to get experimental results that could be compared with the analytical results presented in Section 3.2, the $3^{\text {rd }}$ harmonic was also subtracted from the output current.

The five-phase system is well balanced and first phase is selected for further analysis, as in analytical developments. Different values of $m$ are investigated $(0.2,0.247,0.4$, and $0.494=2 \times 0.247)$, to cover all the possible cases.

In Figs. 3.13, 3.15, 3.17, 3.19, is shown the current ripple obtained with simulations (a) and experiments (b), for different modulation indexes, respectively. The ripple in experiments is processed as explained above and 
also filtered to eliminate the high order harmonics (cut-off frequency $25 \mathrm{kHz}$ ). Comparison is made with the half of the peak-to-peak current ripple, $\tilde{i}_{p p} / 2$. Positive and negative envelope (blue traces) are determined by the equations presented in Section 3.2. The corresponding experimental results are shown as gray traces.

In Figs. 3.14, 3.16, 3.18, 3.20, is depicted the instantaneous output current obtained in simulations (a) and experiments (b) with different modulation indexes, respectively. Current is shown with the calculated upper/lower ripple envelope, depicted in blue colors. The experimental current is shown with gray traces and has not been filtered.

The values of the modulation index have been chosen to cover all possible sub-cases (different colored regions in Fig. 3.5). The agreement is good in the whole fundamental period, proving the effectiveness of the analytical developments.

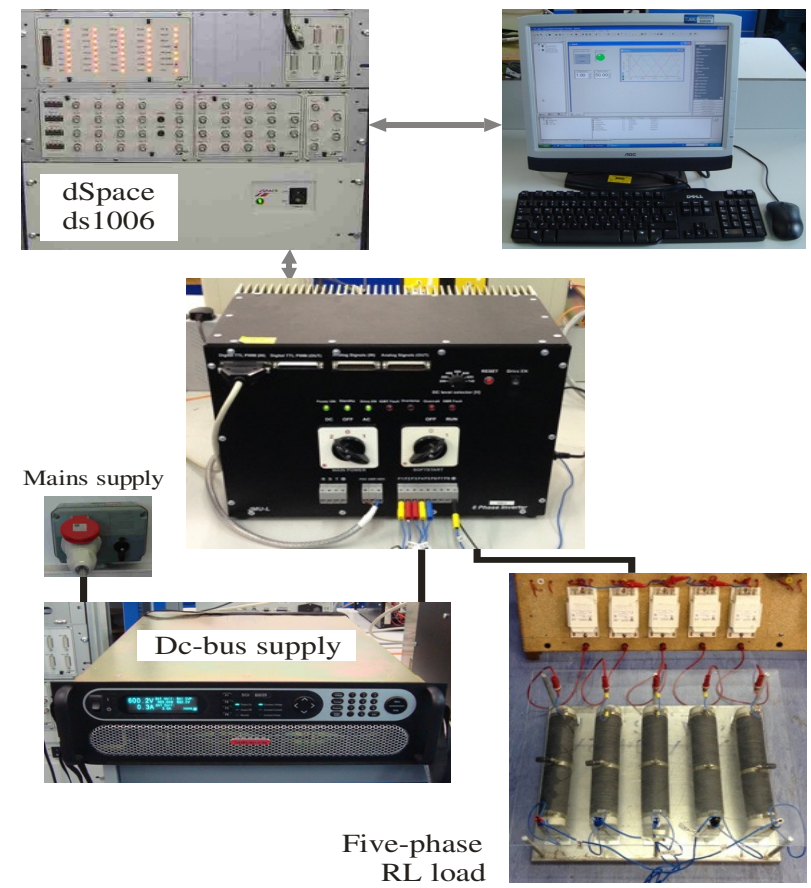

Fig. 3.12. Experimental setup 
(a)

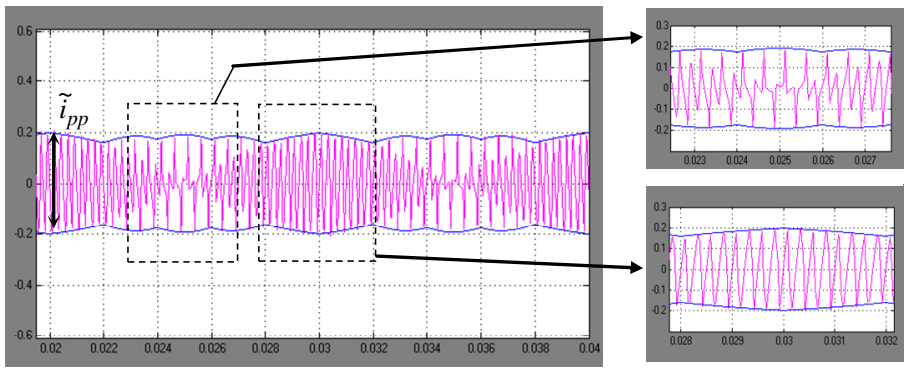

(b)

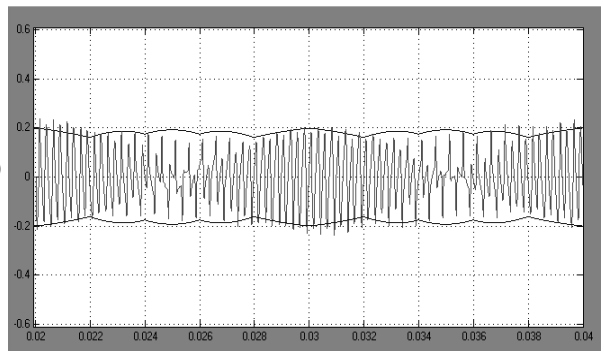

Fig. 3.13. Current ripple (pink), $m=0.2$, and evaluated peak-to-peak envelope (blue) for one fundamental period: (a) simulation, with details, and (b) experimental results.

(a)

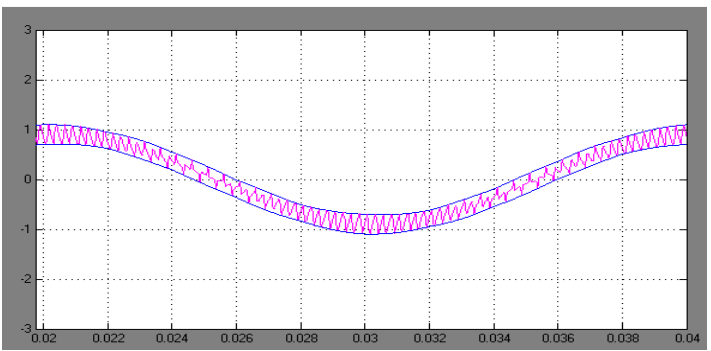

(b)

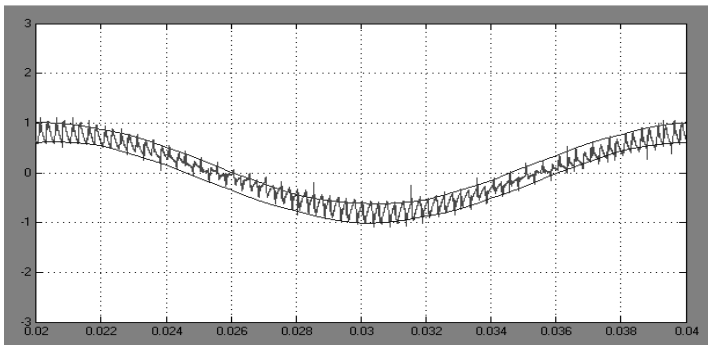

Fig. 3.14. Instantaneous output current (pink) with calculated current envelopes (blue traces) for $m=0.2$ : (a) simulated, (b) experimental results. 
(a)

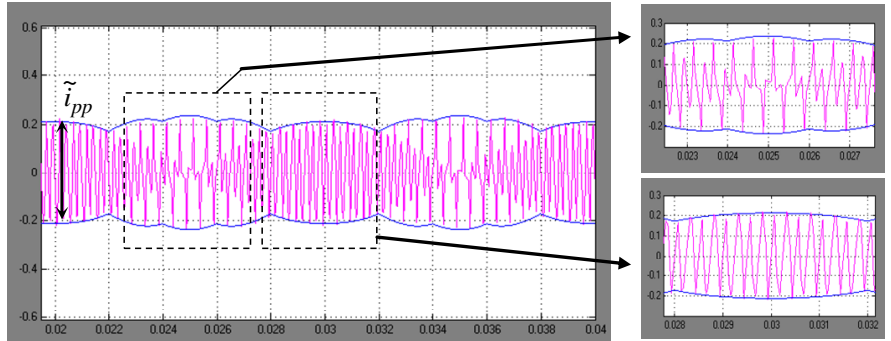

(b)

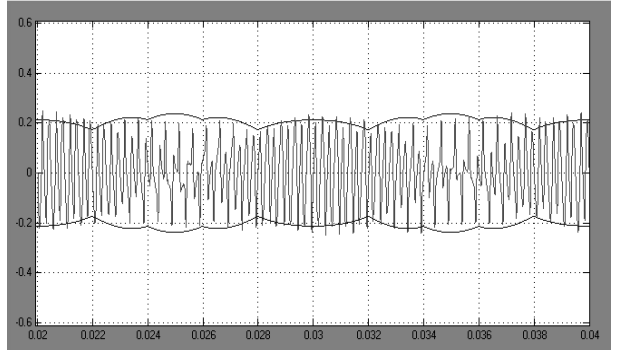

Fig. 3.15. Current ripple (pink), $m=0.247$, and evaluated peak-to-peak envelope (blue) for one fundamental period: (a) simulation, with details, and (b) experimental results.

(a)

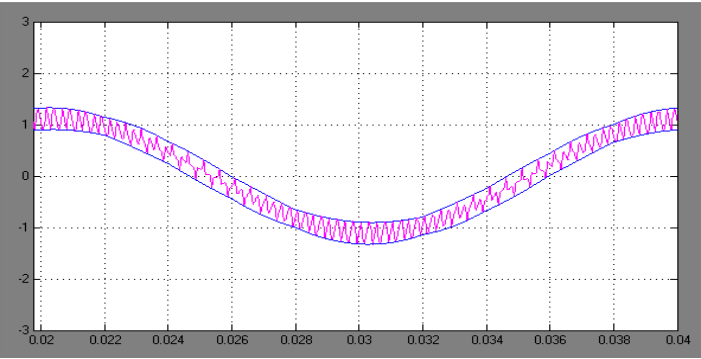

(b)

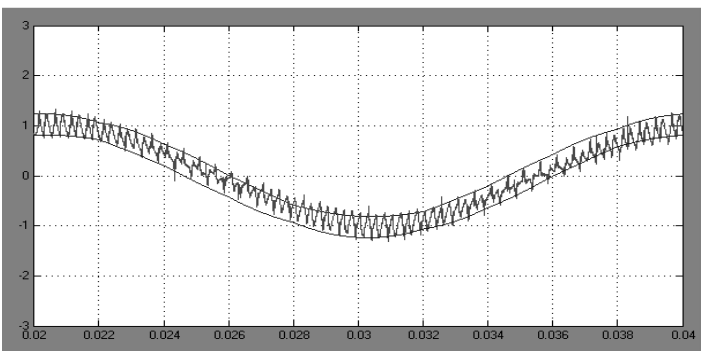

Fig. 3.16. Instantaneous output current (pink) with calculated current envelopes (blue traces) for $m=0.247$ : (a) simulated, (b) experimental results. 
(a)
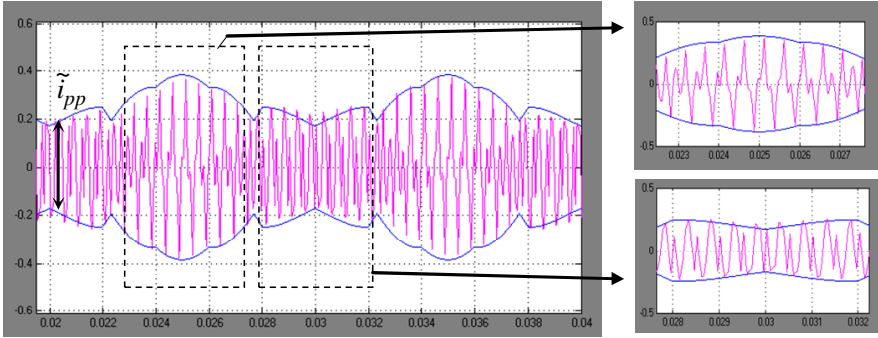

(b)

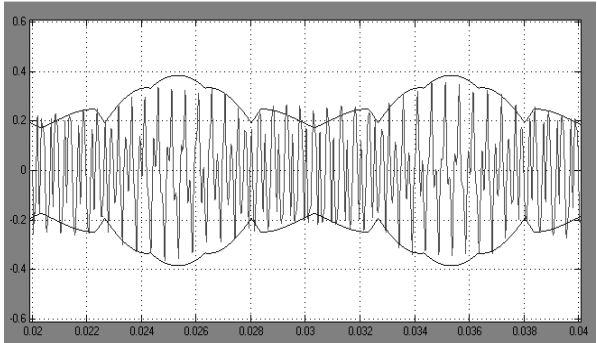

Fig. 3.17. Current ripple (pink), $m=0.4$, and evaluated peak-to-peak envelope (blue) for one fundamental period: (a) simulation, with details, and (b) experimental results.

(a)

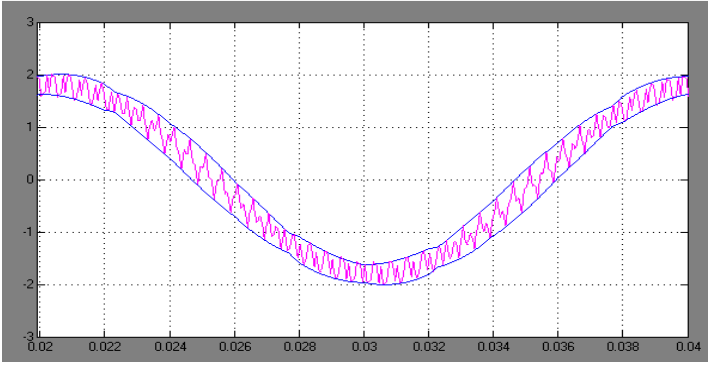

(b)

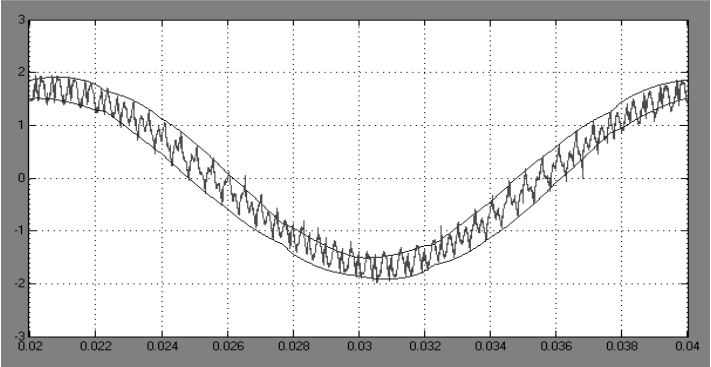

Fig. 3.18. Instantaneous output current (pink) with calculated current envelopes (blue traces) for $m=0.4$ : (a) simulated, (b) experimental results. 
(a)
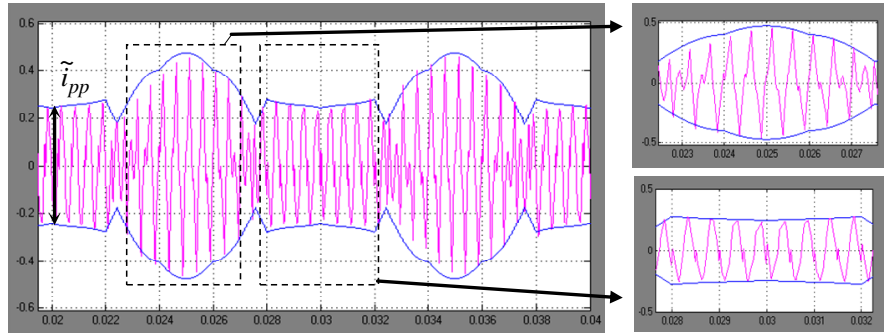

(b)

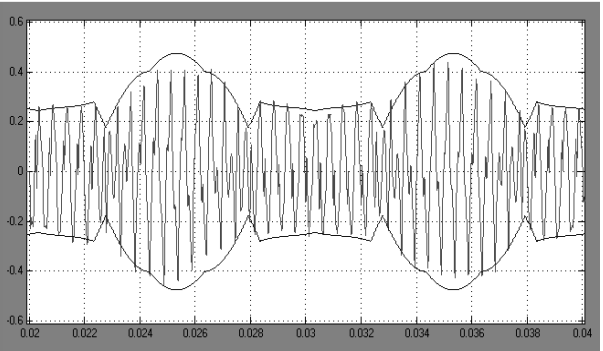

Fig. 3.19. Current ripple (pink), $m=0.494$, and evaluated peak-to-peak envelope (blue) for one fundamental period: (a) simulation, with details, and (b) experimental results.

(a)

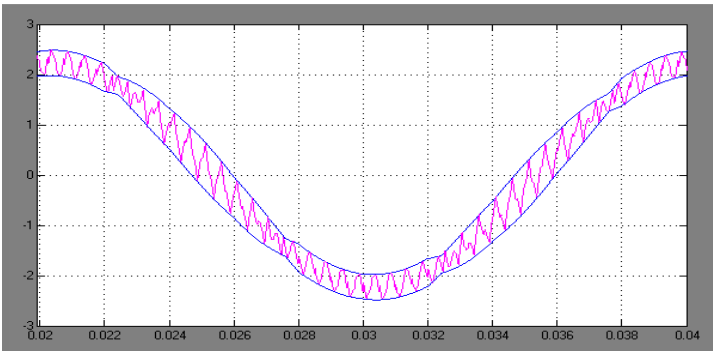

(b)

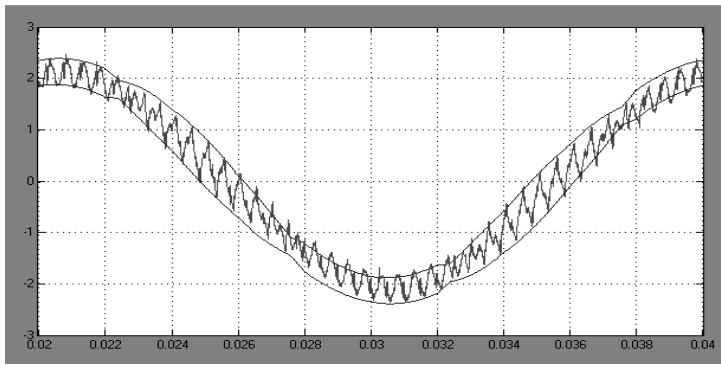

Fig. 3.20. Instantaneous output current (pink) with calculated current envelopes (blue traces) for $m=0.494$ : (a) simulated, (b) experimental results. 


\subsection{Extension of the peak-to-peak output current ripple analysis to seven-phase VSIs}

\subsubsection{State of the art}

Recent studies about RMS output current ripple in multiphase motor drives are presented in [18-21], with emphasis on a five-phase system. In [19] is investigated the output current ripple RMS for the multiphase inverters, emphasis was made on five-phase VSI, by considering the space vector theory and both 2-D planes that characterize the five-phase system. It is shown that output current ripple RMS cannot be minimized by injection of fifth harmonic and its odd multiples, but it is also pointed out that, from the practical point of view, differences in current ripple RMS are relatively small considering sinusoidal PWM and SV-PWM. The principles of the space vector approach, detailed for the five-phase system, and analyzing the deviation of the harmonic flux on two planes, can be directly extended to higher phase numbers. For example, in a seven-phase system, should be considered the three planes, with the same characteristics for all continuous PWM methods. In [22] is presented the use of polygon approach for the analytical analysis of the output currentripple RMS in multiphase drives. Polygon approach appears to be a much simpler and faster analytical tool for the analysis of the output current ripple than space-vector approach. The peak-to-peak output current ripple analysis, in addition to the RMS analysis, has been introduced for the multiphase inverters in [24].

\subsubsection{Calculation of the peak-to-peak output current ripple}

The analysis of seven-phase inverters by the space vector transformation leads to better understanding and more simple calculation of voltage levels and corresponding application times, as in the case of five-phase inverters. In the following analysis is considered the centered PWM, as described in Sect. 3.1.1. The instantaneous current ripple is introduced for a generic balanced sevenphase $R-L-E M F$ load (RLE). The analysis can be extended to seven-phase ac motor drives as well, but the circuit model of the specific ac motor must be carefully considered to evaluate the cases in which it can be reduced to the basic RLE equivalent circuit [19, 22]. 
Multiple space vectors are introduced to represent voltage and current phase quantities in multiphase systems [7]. For the seven-phase system $\left\{x_{1}, x_{2}, x_{3}, x_{4}\right.$, $\left.x_{5}, x_{6}, x_{7}\right\}$, the three space vectors $\boldsymbol{x}_{1}, \boldsymbol{x}_{3}$, and $\boldsymbol{x}_{5}$ lie in the three planes $\alpha_{1}-\beta_{1}, \alpha_{3}-$ $\beta_{3}$ and $\alpha_{5}-\beta_{5}$, respectively, and are expressed as:

$$
\left\{\begin{array}{l}
x_{1}=\frac{2}{7}\left[x_{1}+x_{2} \boldsymbol{\alpha}+x_{3} \boldsymbol{\alpha}^{2}+x_{4} \boldsymbol{\alpha}^{3}+x_{5} \boldsymbol{\alpha}^{4}+x_{6} \boldsymbol{\alpha}^{5}+x_{7} \boldsymbol{\alpha}^{6}\right] \\
\boldsymbol{x}_{3}=\frac{2}{7}\left[x_{1}+x_{2} \boldsymbol{\alpha}^{3}+x_{3} \boldsymbol{\alpha}^{6}+x_{4} \boldsymbol{\alpha}^{2}+x_{5} \boldsymbol{\alpha}^{5}+x_{6} \boldsymbol{\alpha}+x_{7} \boldsymbol{\alpha}^{4}\right] \\
\boldsymbol{x}_{5}=\frac{2}{7}\left[x_{1}+x_{2} \boldsymbol{\alpha}^{5}+x_{3} \boldsymbol{\alpha}^{3}+x_{4} \boldsymbol{\alpha}+x_{5} \boldsymbol{\alpha}^{6}+x_{6} \boldsymbol{\alpha}^{4}+x_{7} \boldsymbol{\alpha}^{2}\right] \\
x_{0}=\frac{1}{7}\left[x_{1}+x_{2}+x_{3}+x_{4}+x_{5}+x_{6}+x_{7}\right]
\end{array}\right.
$$

being $\boldsymbol{\alpha}=\exp (j 2 \pi / 7)$ and $x_{0}$ the zero-sequence component, always null in case of balanced systems.

The inverse transformation of (3.33) is

$$
x_{k}=x_{0}+\boldsymbol{x}_{1} \cdot \boldsymbol{\alpha}^{(k-1)}+\boldsymbol{x}_{3} \cdot \boldsymbol{\alpha}^{3(k-1)}+\boldsymbol{x}_{5} \cdot \boldsymbol{\alpha}^{5(k-1)}, k=1,2, \ldots, 7 .
$$

With reference to a seven-phase VSI supplied by the dc voltage $V_{d c}$, the output space voltage vectors can be written as function of the seven switching leg states $S_{k}=[0,1]$ as

$$
\left\{\begin{array}{l}
v_{1}=\frac{2}{7} V_{d c}\left[S_{1}+S_{2} \boldsymbol{\alpha}+S_{3} \boldsymbol{\alpha}^{2}+S_{4} \boldsymbol{\alpha}^{3}+S_{5} \boldsymbol{\alpha}^{4}+S_{6} \boldsymbol{\alpha}^{5}+S_{7} \boldsymbol{\alpha}^{6}\right] \\
v_{3}=\frac{2}{7} V_{d c}\left[S_{1}+S_{2} \boldsymbol{\alpha}^{3}+S_{3} \boldsymbol{\alpha}^{6}+S_{4} \boldsymbol{\alpha}^{2}+S_{5} \boldsymbol{\alpha}^{5}+S_{6} \boldsymbol{\alpha}+S_{7} \boldsymbol{\alpha}^{4}\right] \\
v_{5}=\frac{2}{7} V_{d c}\left[S_{1}+S_{2} \boldsymbol{\alpha}^{5}+S_{3} \boldsymbol{\alpha}^{3}+S_{4} \boldsymbol{\alpha}+S_{5} \boldsymbol{\alpha}^{6}+S_{6} \boldsymbol{\alpha}^{4}+S_{7} \boldsymbol{\alpha}^{2}\right]
\end{array}\right.
$$

The space vector diagrams representing all possible switch configurations on planes $\alpha_{1}-\beta_{1}, \alpha_{3}-\beta_{3}$ and $\alpha_{5}-\beta_{5}$ are given in Fig. 3.4.

The SV-PWM of seven-phase inverters is based on the determination of application times of active and null inverter voltage vectors $\boldsymbol{v}_{1}, \boldsymbol{v}_{3}$, and $\boldsymbol{v}_{5}$ in every switching period $T_{s}$. In the case of symmetrical SV-PWM, the sequence is determined in $T_{s} / 2$ and it is repeated symmetrically in the next half of the switching period. Also in the case of seven-phase inverters, by equally sharing 
the application time of the zero voltage vector between the null switch configurations 0000000 and 1111111, the centered PWM is realized, able to minimize the RMS of current ripple. As already mentioned, this SV-PWM provides the same switching pattern such as the CB-PWM when a "min/max centered" common-mode voltage is injected into the modulating signals [12]. As result of the SV-PWM, for each phase, the average of the inverter output voltage $\bar{v}\left(T_{s}\right)$ corresponds to the reference voltage $v^{*}$.

In the case of sinusoidal balanced output voltages supplying a balanced load, the zero-sequence component is null. Introducing the modulation index $\mathrm{m}$ $=V^{*} / V_{d c}$ the reference space voltage vectors become

$$
\left\{\begin{array}{l}
\boldsymbol{v}_{1}^{*}=\boldsymbol{v}^{*}=m V_{d c} e^{j \vartheta} \\
\boldsymbol{v}_{3}^{*}=0 \\
\boldsymbol{v}_{5}^{*}=0 .
\end{array}\right.
$$

In this case, SVM is quarter-wave symmetric, and it can be analyzed in the range $[0, \pi / 2]$ of the phase angle $\vartheta=\omega t$. With reference to Fig. 3.21, the three sectors (1), (2), and (3) are considered for $0 \leq \vartheta \leq \pi / 7, \pi / 7 \leq \vartheta \leq 2 \pi / 7$, and $2 \pi / 7$

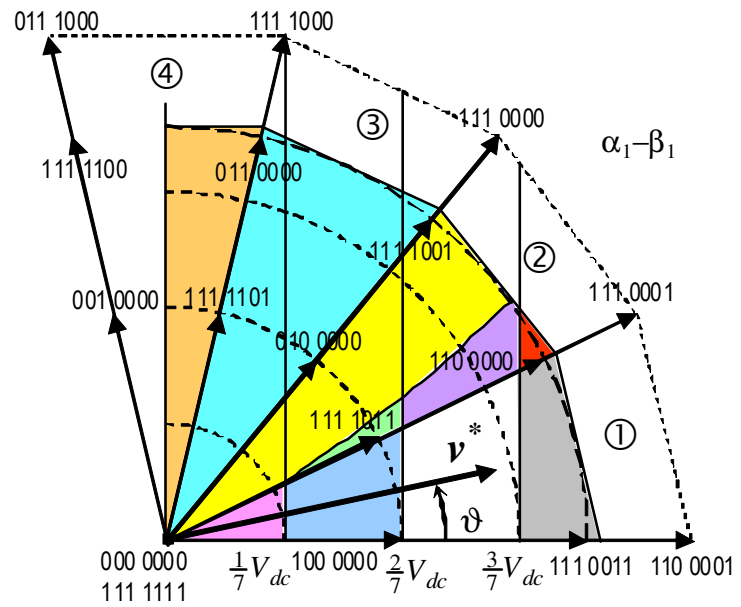

Fig. 3. 21. Space vector diagram of inverter output voltage on plane $\alpha_{1}-\beta_{1}$ in the range $\vartheta=[0$, $90^{\circ}$ ]. Outer dashed circle is modulation limit, $m_{\max } \approx 0.513$. Different colored areas correspond to different equations for determining the current ripple. 
$\leq \vartheta \leq 3 \pi / 7$, respectively, and the half of sector (4) is considered for $3 \pi / 7 \leq \vartheta \leq$ $\pi / 2$.

For sector (1) the application times of the switch configurations involved in the modulation sequence from $\{00\}$ to $\{7 \mathrm{~F}\}$ (hexadecimal) can be determined in the half period $T_{s} / 2$ as [12]

$$
\begin{aligned}
& t_{1}=m T_{s} K_{1} \sin (\pi / 7-\vartheta), \quad 1000000=\{40\} \\
& t_{2}=m T_{s} K_{5} \sin \vartheta, \quad 1100000=\{60\} \\
& t_{3}=m T_{s} K_{3} \sin (\pi / 7-\vartheta), \quad 1100001=\{61\} \\
& t_{4}=m T_{s} K_{3} \sin \vartheta, \quad 1110001=\{71\} \\
& t_{5}=m T_{s} K_{5} \sin (\pi / 7-\vartheta), \quad 1110011=\{73\} \\
& t_{6}=m T_{s} K_{1} \sin \vartheta, \quad 1111011=\{7 \mathrm{~B}\} \\
& t_{0}=\frac{T_{s}}{2}-\left(t_{1}+t_{2}+t_{3}+t_{4}+t_{5}+t_{6}\right)= \\
& =1-2 m\left(K_{1}+K_{3}+K_{5}\right)\left[K_{1} \cos \vartheta+\left(1-\cos \frac{\pi}{7}\right) \sin \vartheta\right] \text {, } \\
& \left(\begin{array}{ll}
000 & 0000 \\
111 & 1111
\end{array}\right)=\left\{\begin{array}{l}
00 \\
7 \mathrm{~F}
\end{array}\right\} \\
& \left\{\begin{array}{l}
K_{1}=\sin \frac{\pi}{7} \cong 0.434 \\
K_{3}=\sin \frac{3 \pi}{7} \cong 0.975 \\
K_{5}=\sin \frac{5 \pi}{7} \cong 0.782 .
\end{array}\right.
\end{aligned}
$$

Equations (3.37)-(3.42) can be extended to any sector $k$ by replacing the phase angle $\vartheta$ with $\vartheta-(k-1) \pi / 7, k=1,2, \ldots, 14$.

Due to the symmetry among all phases in the considered case of sinusoidal balanced currents, only the first phase is examined in the following analysis. In terms of multiple space vectors, the phase variables are given by (3.34). For the 
first phase, it results in the projection of the three space vectors on the real axes. In particular, introducing (3.36) in (3.34), the average output voltage of the first phase is given by

$$
\bar{v}\left(T_{s}\right)=v^{*}=\mathfrak{R} e\left\{\boldsymbol{v}_{1}^{*}\right\}+\mathfrak{R} e\left\{\boldsymbol{v}_{3}^{*}\right\}+\mathfrak{R} e\left\{\boldsymbol{v}_{5}^{*}\right\}=m V_{d c} \cos \vartheta .
$$

By introducing (3.44) in the expression for the alternating voltage (2.6), and calculating $v(t)$ by (3.34) and (3.35), the alternating component of inverter output voltage (first phase) can be written as

$$
\tilde{v}(t)=\left[S_{1}-\frac{1}{7}\left(S_{1}+S_{2}+S_{3}+S_{4}+S_{5}+S_{6}+S_{7}\right)\right] V_{d c}-m V_{d c} \cos \vartheta
$$

In order to evaluate the current ripple in the whole phase angle range $0<$ $\vartheta<\pi / 2$, the four main cases corresponding to the four sectors depicted in Fig. 3.21 should be considered. Additional sub-cases, also determined by the value of modulation index, are identified in Fig. 3.21 with different colored areas.

The current ripple for each sector is depicted in a separate diagram, Figs. 3.22 and 3.23. In general, for all the four sectors, the ripple shows two different peaks in the switching period (two positive peaks and the symmetric negative peaks). In most of these cases, one peak results to be always bigger than the other in the considered sector, for any specific range of $m \cos \vartheta$, as a consequence of both current slopes and application times. There are only 2 exceptions, both related to the second sector (2). For these cases, either one or the other peak is bigger depending on the values of $m$ and $\vartheta$. These aspects are addressed with more details in the following.

1) Evaluation in the first sector

Considering sector (1), $0 \leq \vartheta \leq \pi / 7$, four different sub-cases can be distinguished: $0 \leq m \cos \vartheta \leq 1 / 7,1 / 7 \leq m \cos \vartheta \leq 2 / 7,2 / 7 \leq m \cos \vartheta \leq 3 / 7$, and $3 / 7$ $\leq m \cos \vartheta \leq m_{\max } \cos \vartheta<4 / 7$. All sub-cases are represented in Fig. 3.22a.

The sub-case $0 \leq m \cos \vartheta \leq 1 / 7$ (pink area in Fig. 3.21) is considered in diagram (1) of Fig. 3.22a, where the current ripple $\tilde{i}$ and its peak-to-peak value $\tilde{i}_{p p}$ are depicted, together with the instantaneous output voltage $v(t)$. In this case, according to Fig. 3.22a, $\tilde{i}_{p p}$ can be evaluated by the expressions for the instantaneous current ripple and its peak-to-peak value, (2.10) and (2.11), and 

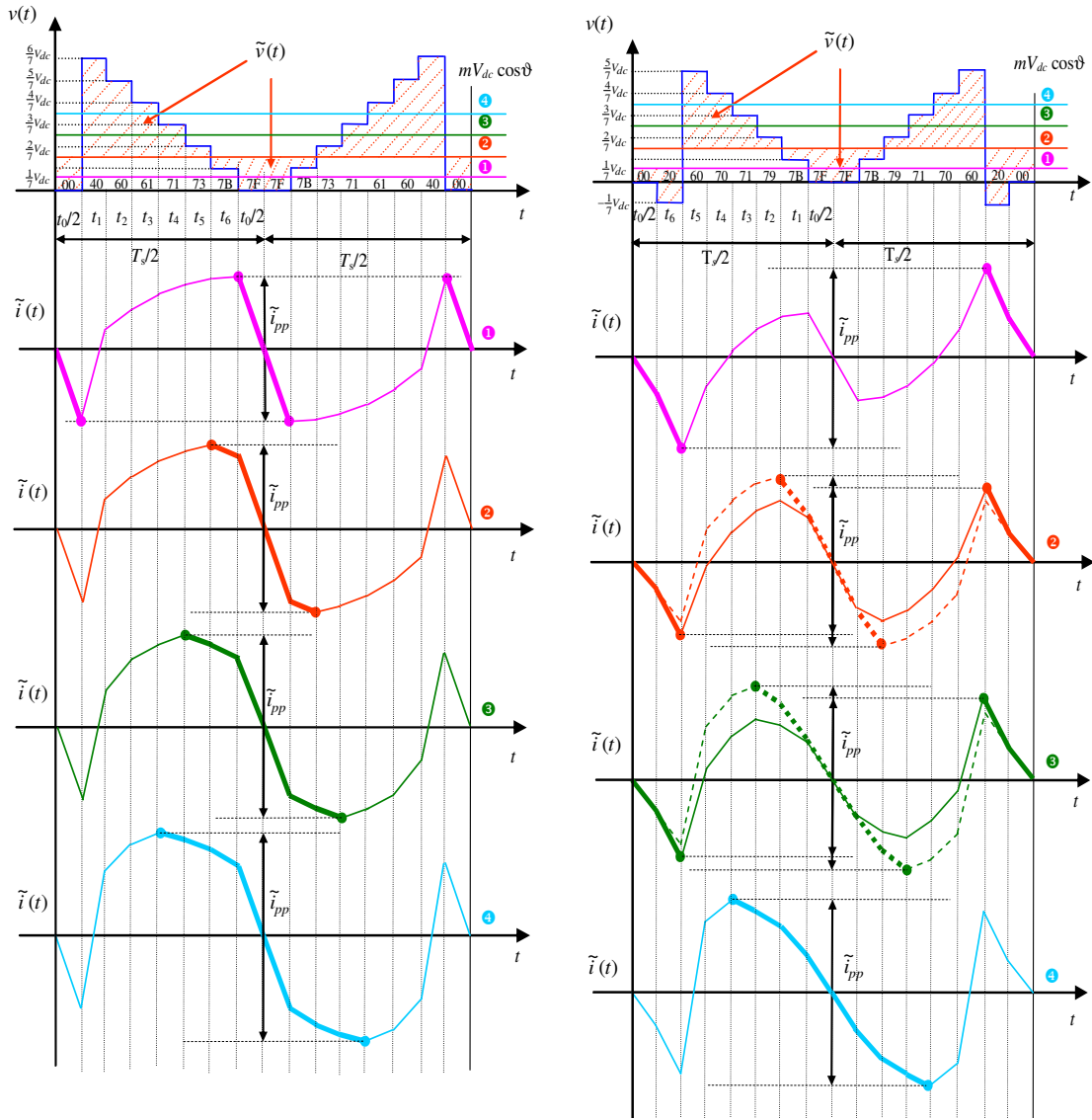

Fig. 3.22. Output voltage and current ripple in a switching period: (a) for sector (1), $0 \leq \theta \leq \pi / 7$, and (b) for sector (2), $\pi / 7 \leq \theta \leq 2 \pi / 7$.

(3.45) considering the switch configurations $\{00\}$ or $\{7 \mathrm{~F}\}$ with the corresponding application interval $t_{0}$, leading to:

$$
\tilde{i}_{p p}=\frac{1}{L}\left\{m V_{d c} \cos \vartheta t_{0}\right\} .
$$

Considering the same normalization as for five-phase inverters (3.14) and introducing the duty-cycle $\delta_{0}=t_{0} / T_{s} / 2$, normalized current ripple can be determined as 


$$
r(m, \vartheta)=m \cos \vartheta \delta_{0} .
$$

The sub-case $1 / 7 \leq m \cos \vartheta \leq 2 / 7$ (azure area in Fig. 3.21) is depicted in diagram 2 of Fig. 3.22a. In this case $\tilde{i}_{p p}$ can be evaluated considering the switch configurations $\{7 \mathrm{~F}\}$ and $\{7 \mathrm{~B}\}$, with the corresponding application intervals $t_{0} / 2$ and $t_{6}$, leading to

$$
\tilde{i}_{p p}=\frac{2}{L}\left\{m V_{d c} \cos \vartheta \frac{t_{0}}{2}+\left(m V_{d c} \cos \vartheta-\frac{V_{d c}}{7}\right) t_{6}\right\} .
$$

Normalizing by (3.14) and introducing the duty-cycles $\delta_{k}=t_{k} / T_{s} / 2$, the normalized current ripple becomes

$$
r(m, \vartheta)=m \cos \vartheta \delta_{0}+2\left(m \cos \vartheta-\frac{1}{7}\right) \delta_{6} .
$$

The sub-case $2 / 7 \leq m \cos \vartheta \leq 3 / 7$ (white area in Fig. 3.21) is depicted in diagram 3 of Fig. 3.22a. In this case $\tilde{i}_{p p}$ can be evaluated considering the switch configurations $\{7 \mathrm{~F}\},\{7 \mathrm{~B}\}$, and $\{73\}$ with the corresponding application intervals $t_{0} / 2, t_{6}$, and $t_{5}$, leading to

$$
\tilde{i}_{p p}=\frac{2}{L}\left\{m V_{d c} \cos \vartheta \frac{t_{0}}{2}+\left(m V_{d c} \cos \vartheta-\frac{V_{d c}}{7}\right) t_{6}+\left(m V_{d c} \cos \vartheta-\frac{2 V_{d c}}{7}\right) t_{5}\right\} \text {. }
$$

The corresponding normalized current ripple is

$$
r(m, \vartheta)=m \cos \vartheta \delta_{0}+2\left(m \cos \vartheta-\frac{1}{7}\right) \delta_{6}+2\left(m \cos \vartheta-\frac{2}{7}\right) \delta_{5} .
$$

The sub-case $3 / 7 \leq m \cos \vartheta \leq m_{\max } \cos \vartheta<4 / 7$ (gray area in Fig. 3.21) is depicted in diagram 4 of Fig. 3.22a. In this case $\widetilde{i}_{p p}$ can be evaluated considering the switch configurations $\{7 \mathrm{~F}\},\{7 \mathrm{~B}\},\{73\}$, and $\{71\}$ with the corresponding application intervals $t_{0} / 2, t_{6}, t_{5}$, and $t_{4}$, leading to

$$
\begin{aligned}
\tilde{i}_{p p}= & \frac{2}{L}\left\{m V_{d c} \cos \vartheta \frac{t_{0}}{2}+\left(m V_{d c} \cos \vartheta-\frac{V_{d c}}{7}\right) t_{6}+\left(m V_{d c} \cos \vartheta-\frac{2 V_{d c}}{7}\right) t_{5}+\right. \\
& \left.+\left(m V_{d c} \cos \vartheta-\frac{3 V_{d c}}{7}\right) t_{4}\right\}
\end{aligned}
$$


The corresponding normalized current ripple is

$$
r(m, \vartheta)=m \cos \vartheta \delta_{0}+2\left(m \cos \vartheta-\frac{1}{7}\right) \delta_{6}+2\left(m \cos \vartheta-\frac{2}{7}\right) \delta_{5}+2\left(m \cos \vartheta-\frac{3}{7}\right) \delta_{4} .
$$

2) Evaluation in the second sector

Considering the sector (2), $\pi / 7 \leq \vartheta \leq 2 \pi / 7$, four different sub-cases can be identified: $0 \leq m \cos \vartheta \leq 1 / 7,1 / 7 \leq m \cos \vartheta \leq 2 / 7,2 / 7 \leq m \cos \vartheta \leq 3 / 7$, and $3 / 7 \leq$ $m \cos \vartheta \leq m_{\max } \cos \vartheta<4 / 7$. All these sub-cases are represented in Fig. 3.22b.

The sub-case $0 \leq m \cos \vartheta \leq 1 / 7$ (yellow area in Fig. 3.21) is depicted in diagram 10 of Fig. 3.22b. According to this figure, $\widetilde{i}_{p p}$ can be evaluated by (2.10), (2.11) and (3.45) considering the switch configurations $\{00\}$ and $\{20\}$ with the corresponding application intervals $t_{0} / 2$ and $t_{6}$, leading to

$$
\tilde{i}_{p p}=\frac{2}{L}\left\{m V_{d c} \cos \vartheta \frac{t_{0}}{2}+\left(m V_{d c} \cos \vartheta+\frac{1}{7} V_{d c}\right) t_{6}\right\} .
$$

Normalizing by (3.14) and introducing the duty-cycles, the normalized peak-to-peak current ripple amplitude becomes:

$$
r(m, \vartheta)=m \cos \vartheta \delta_{0}+2\left(m \cos \vartheta+\frac{1}{7}\right) \delta_{6} .
$$

The sub-case $1 / 7 \leq m \cos \vartheta \leq 2 / 7$ is depicted in diagram 2 of Fig. 3.22b. It can be noticed that there are two possible situations regarding the evaluation of $\tilde{i}_{p p}$, corresponding to yellow-green area of sector (2) in Fig. 3.21:

- in the first situation, yellow area (solid orange line in Fig. $3.22 \mathrm{~b}$ ), $\tilde{i}_{p p}$ can be determined as in the previous sub-case by considering the switch configurations $\{00\}$ and $\{20\}$ with the corresponding application intervals $t_{0} / 2$ and $t_{6}$, leading to (3.54) and (3.55);

- in the second situation, green area (dashed orange line in Fig. 3.22b), $\tilde{i}_{p p}$ can be determined by considering the switch configurations $\{7 \mathrm{~F}\}$ and $\{7 \mathrm{~B}\}$ with the corresponding application intervals $t_{0} / 2$ and $t_{1}$, leading to

$$
\tilde{i}_{p p}=\frac{2}{L}\left\{m V_{d c} \cos \vartheta \frac{t_{0}}{2}+\left(m V_{d c} \cos \vartheta-\frac{1}{7} V_{d c}\right) t_{1}\right\},
$$

being the normalized peak-to-peak current ripple amplitude 


$$
r(m, \vartheta)=m \cos \vartheta \delta_{0}+2\left(m \cos \vartheta-\frac{1}{7}\right) \delta_{1}
$$

The sub-case $2 / 7 \leq m \cos \vartheta \leq 3 / 7$ is depicted in diagram 3 of Fig. 3.22b. Also for this sub-case there are two possible situations for evaluating, $\tilde{i}_{p p}$ corresponding to yellow-violet area of sector (2) in Fig. 3.21:

- in the first situation, yellow area (solid green line in Fig. $3.22 \mathrm{~b}$ ), $\tilde{i}_{p p}$ can be determined as in the previous sub-case by considering the switch configurations $\{00\}$ and $\{20\}$ with the corresponding application intervals $t_{0} / 2$ and $t_{6}$, leading to (3.54) and (3.55);

- in the second situation, violet area (dashed green line in Fig. 3.22b), $\tilde{i}_{p p}$ can be determined by considering the switch configurations $\{7 \mathrm{~F}\},\{7 \mathrm{~B}\}$, and $\{79\}$, with the corresponding application intervals $t_{0} / 2, t_{1}$, and $t_{2}$, leading to:

$$
\tilde{i}_{p p}=\frac{2}{L}\left\{m V_{d c} \cos \vartheta \frac{t_{0}}{2}+\left(m V_{d c} \cos \vartheta-\frac{V_{d c}}{7}\right) t_{1}+\left(m V_{d c} \cos \vartheta-\frac{2 V_{d c}}{7}\right) t_{2}\right\},
$$

being the normalized current ripple amplitude

$$
r(m, \vartheta)=m \cos \vartheta \delta_{0}+2\left(m \cos \vartheta-\frac{1}{7}\right) \delta_{1}+2\left(m \cos \vartheta-\frac{2}{7}\right) \delta_{2} .
$$

The last sub-case $3 / 7 \leq m \cos \vartheta \leq m_{\max } \cos \vartheta<4 / 7$ (red area in Fig. 3.21) is depicted in diagram 4 of Figure $3.22 \mathrm{~b}$. According to this figure, $\widetilde{i}_{p p}$ can be evaluated considering the switch configurations $\{7 \mathrm{~F}\},\{7 \mathrm{~B}\},\{79\}$, and $\{71\}$ with the corresponding application intervals $t_{0} / 2, t_{1}, t_{2}$, and $t_{3}$, leading to

$$
\begin{aligned}
\tilde{i}_{p p}= & \frac{2}{L}\left\{m V_{d c} \cos \vartheta \frac{t_{0}}{2}+\left(m V_{d c} \cos \vartheta-\frac{V_{d c}}{7}\right) t_{1}+\left(m V_{d c} \cos \vartheta-\frac{2 V_{d c}}{7}\right) t_{2}+\right. \\
& \left.+\left(m V_{d c} \cos \vartheta-\frac{3 V_{d c}}{7}\right) t_{3}\right\} .
\end{aligned}
$$

Normalized current ripple is $r(m, \vartheta)=m \cos \vartheta \delta_{0}+2\left(m \cos \vartheta-\frac{1}{7}\right) \delta_{1}+2\left(m \cos \vartheta-\frac{2}{7}\right) \delta_{2}+2\left(m \cos \vartheta-\frac{3}{7}\right) \delta_{3}$. 


\section{3) Evaluation in the third sector}

With reference to sector (3), $2 \pi / 7 \leq \vartheta \leq 3 \pi / 7$ (turquoise area in Fig. 3.21), three different ranges can be distinguished: $0 \leq m \cos \vartheta \leq 1 / 7,1 / 7 \leq m \cos \vartheta \leq$ $2 / 7$, and $2 / 7 \leq m \cos \vartheta \leq m_{\max } \cos \vartheta<3 / 7$. All the sub-cases are represented in diagrams (1, 2, 3 of Fig. 3.23a. It can be noted that there are not sub-cases, and for all the three ranges $\tilde{i}_{p p}$ can be evaluated considering the switch configurations $\{00\},\{20\}$, and $\{30\}$ with the corresponding application intervals $t_{0}, t_{1}$, and $t_{2}$, leading to

$\tilde{i}_{p p}=\frac{2}{L}\left\{m V_{d c} \cos \vartheta \frac{t_{0}}{2}+\left(m V_{d c} \cos \vartheta+\frac{V_{d c}}{7}\right) t_{1}+\left(m V_{d c} \cos \vartheta+\frac{2 V_{d c}}{7}\right) t_{2}\right\}$.

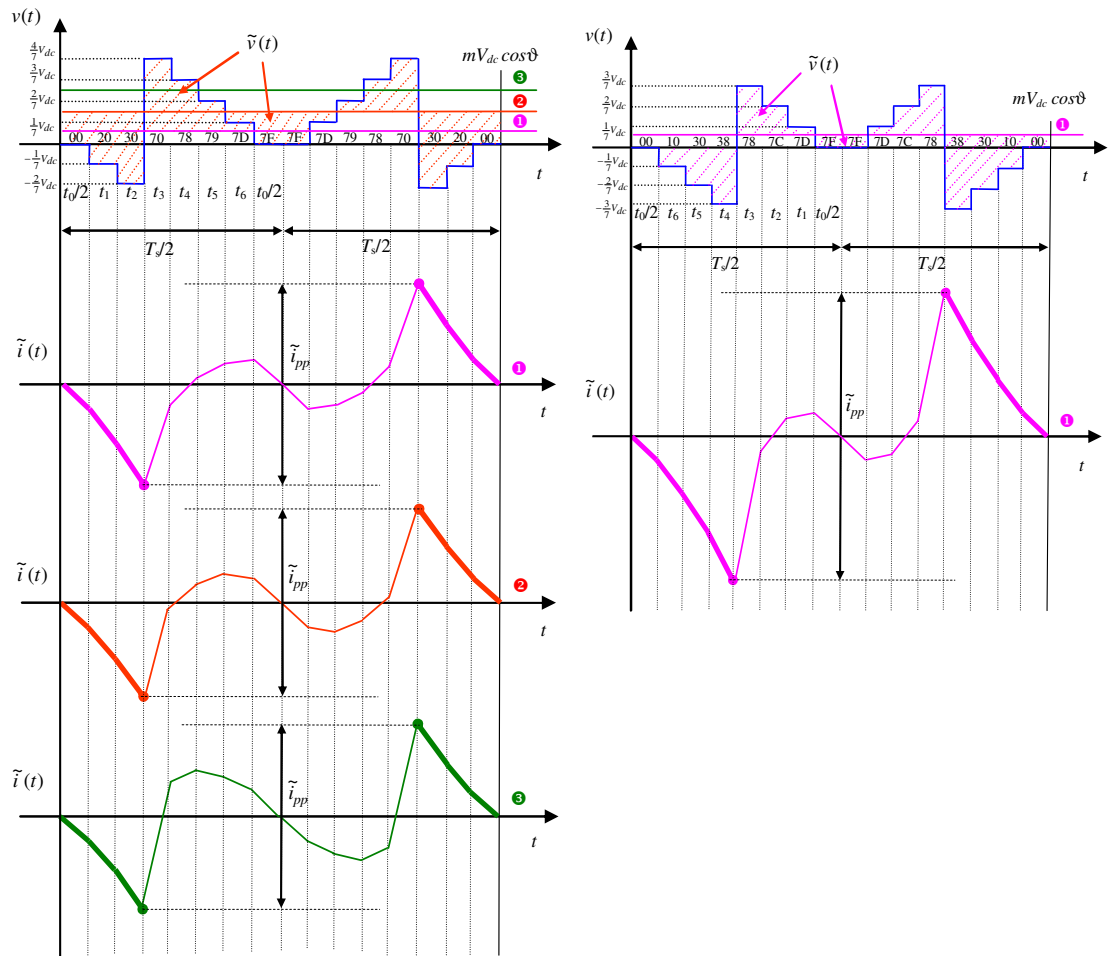

Fig. 3.23. Output voltage and current ripple in a switching period: (a) for sector 3 , $2 \pi / 7 \leq \theta \leq$ $3 \pi / 7$, and (b) for sector (4), $3 \pi / 7 \leq \theta \leq \pi / 2$. 
Normalized current ripple is

$$
r(m, \vartheta)=m \cos \vartheta \delta_{0}+2\left(m \cos \vartheta+\frac{1}{7}\right) \delta_{1}+2\left(m \cos \vartheta+\frac{2}{7}\right) \delta_{2} .
$$

4) Evaluation in the fourth (half) sector

With reference to the half of sector (4), $3 \pi / 7 \leq \vartheta \leq \pi / 2$ (orange area in Fig. 3.21 ), there are not sub-cases and the only occurrence is $0 \leq m \cos \vartheta \leq m_{\max }$ $\cos \vartheta<1 / 7$, as depicted in Figure 3.23b. In this case, $\tilde{i}_{p p}$ can be evaluated considering the switch configurations $\{00\},\{10\},\{30\}$, and $\{38\}$ with the corresponding application interval $t_{0} / 2, t_{6}, t_{5}$, and $t_{4}$, leading to

$$
\begin{aligned}
\tilde{i}_{p p}= & \frac{2}{L}\left\{m V_{d c} \cos \vartheta \frac{t_{0}}{2}+\left(m V_{d c} \cos \vartheta+\frac{V_{d c}}{7}\right) t_{6}+\left(m V_{d c} \cos \vartheta+\frac{2 V_{d c}}{7}\right) t_{5}+\right. \\
& \left.+\left(m V_{d c} \cos \vartheta+\frac{3 V_{d c}}{7}\right) t_{4}\right\} .
\end{aligned}
$$

The corresponding normalized current ripple is

$$
r(m, \vartheta)=m \cos \vartheta \delta_{0}+2\left(m \cos \vartheta+\frac{1}{7}\right) \delta_{6}+2\left(m \cos \vartheta+\frac{2}{7}\right) \delta_{5}+2\left(m \cos \vartheta+\frac{3}{7}\right) \delta_{4} \cdot
$$

\subsubsection{Peak-to-peak current ripple diagrams}

In order to show the behavior of the peak-to-peak current ripple amplitude in the fundamental period for all the considered cases, in Figs. 3.24 and 3.25 is represented the normalized function $r(m, \vartheta)$ defined by (3.14). Figure 3.24 shows $r(\vartheta)$ for $m=1 / 7,2 / 7,3 / 7$, and $0.513\left(\approx m_{\max }\right)$, corresponding to the dashed circles in Figure 3.21. The four ranges corresponding to the four sectors from (1) to (4) are emphasized. The further sub-regions in sector (2) (green-, violet-, and red-colored areas in Figure 3.21) can be distinguished for $m=2 / 7$ and $3 / 7$.

Figure 3.25 shows the colored map of $r(m, \vartheta)$ for the first quadrant within the modulation limits. It can be noted that ripple amplitude is obviously zero for $m=0$, since the null configurations are the only applied, increasing almost proportionally with $m$ in the neighborhoods of $m=0$. 


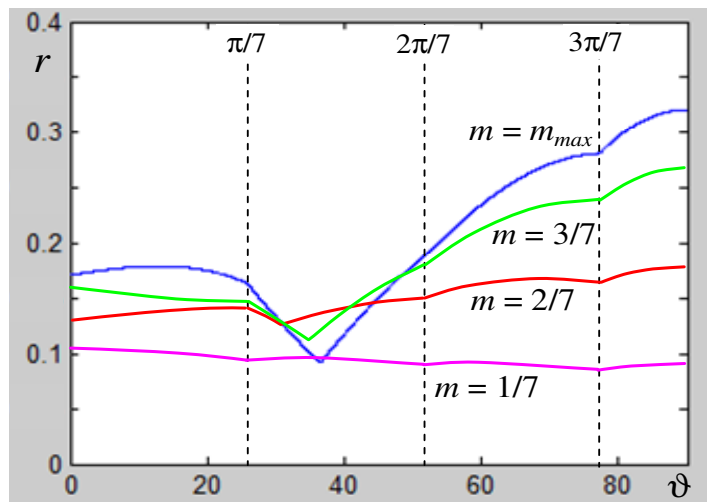

Fig. 3.24. Diagram of the normalized peak-to-peak current ripple amplitude $r(m, \vartheta)$ as a function of the phase angle $\vartheta$ in the range [0, $\pi / 2]$, for different modulation indexes.

A phase angle with minimum ripple can be indentified in the range $\vartheta \approx 30^{\circ} / 35^{\circ}$. A phase angle with maximum ripple is $\vartheta=90^{\circ}$, with ripple amplitude proportional to modulation index: $r\left(m, 90^{\circ}\right)=0.626 \mathrm{~m}$, resulting from (3.65). This aspect is further developed in the following.

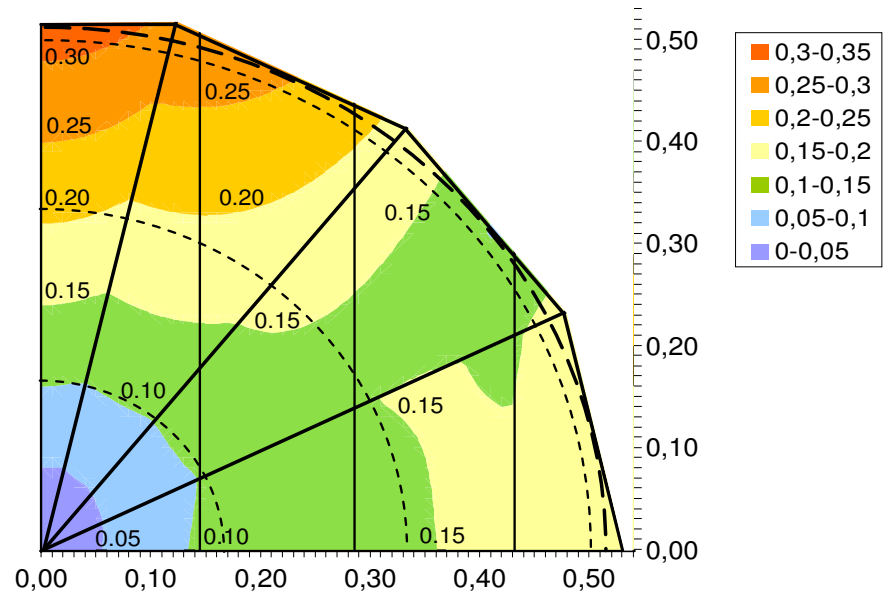

Fig. 3.25. Diagram of the normalized peak-to-peak current ripple amplitude $r(m, \vartheta)$ as colored map in the space vector plane $\alpha-\beta$ within the modulation limits. 


\subsubsection{Maximum of the current ripple}

In order to estimate the current ripple amplitude in the whole fundamental period, the maximum of the current ripple can be evaluated in the phase angle range $\left[0,90^{\circ}\right]$. For this purpose, two relevant angles can be observed in Figs. 3.24 and 3.25: a local maximum is for $\vartheta=0$, and a further local maximum is for $\vartheta=90^{\circ}$. In particular, to determine these two local maxima, $\vartheta=0$ can be set in (3.47), and $\vartheta=90^{\circ}$ in (3.65), introducing the application times given by (3.37-3.42). The maximum value of normalized peak-to-peak current ripple amplitude as a function of the modulation index becomes

$$
r^{\max }(m)=\max \left\{m\left[1-2 m K_{1}^{2}-2 m K_{1} K_{3}-2 m K_{1} K_{5}\right], 0.626 m\right\} .
$$

The intersection between the two local maxima gives the border value of the modulation index

$$
m\left[1-2 m K_{1}^{2}-2 m K_{1} K_{3}-2 m K_{1} K_{5}\right]=0.626 m,
$$

leading to $m \approx 0.197$. Finally, combining (3.66) and (3.67), the maximum of the normalized current ripple is

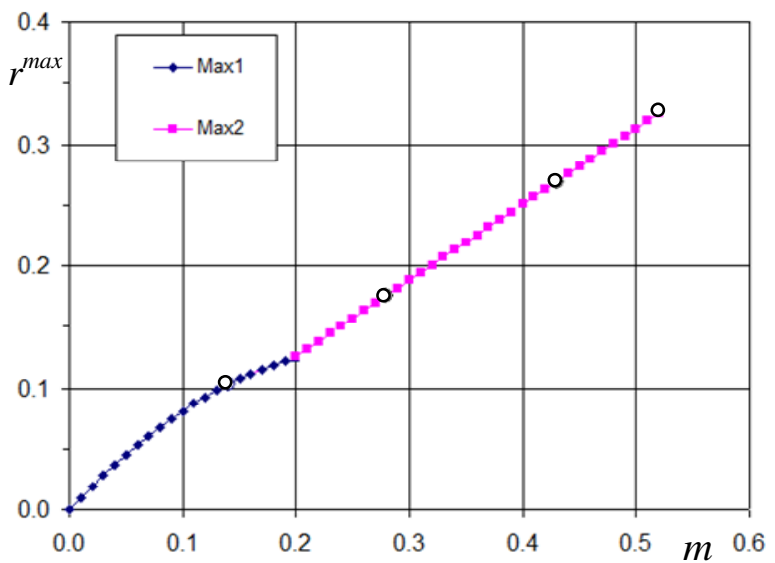

Fig. 3.26. Maximum of the normalized peak-to-peak current ripple amplitude as function of modulation index. 


$$
r^{\max }(m)=\left\{\begin{array}{l}
m\left[1-2 m K_{1}^{2}-2 m K_{1} K_{3}-2 m K_{1} K_{5}\right], m \leq 0.197 \\
0.626 m, m \geq 0.197 .
\end{array}\right.
$$

The composition of the two local maxima is given in Fig. 3.26, leading to the global maximum. The four white dots represent the specific modulation indexes, $m=1 / 7,2 / 7,3 / 7$, and $0.513\left(\approx m_{\max }\right)$, also displayed in Fig. 3.24 and further examined in simulations. It can be noted that maximum function is almost linear with the modulation index, strictly for $m>0.197$. Then, on the basis of (3.68) and (3.14), a simplified expression for maximum of peak-to-peak current ripple amplitude can be obtained for the seven-phase inverter

$$
\tilde{i}_{p p}^{\max } \cong \frac{V_{d c} T_{s}}{3.2 L} m
$$




\subsection{Numerical results}

In order to verify the theoretical developments shown in previous sections, circuit simulations are carried out by Sim-PowerSystems of Matlab considering seven-phase inverter supplying a balanced RL load, having $R=7 \Omega$ and $L=3$ $\mathrm{mH}$. In all simulations the fundamental frequency $f$ is set to $50 \mathrm{~Hz}$, the switching frequency $1 / T_{s}$ is $2.1 \mathrm{kHz}$, and the dc voltage supply $V_{d c}$ is $100 \mathrm{~V}$. A centered symmetrical carrier-based PWM technique is considered, equivalent to centered space vector modulation.

The instantaneous current ripple $\tilde{i}$ is calculated as the difference between the instantaneous current $i(t)$ and its fundamental component $I_{\text {fund }}(t)$, i.e.:

$$
\tilde{i}(t)=i(t)-I_{\text {fund }}(t) \text {. }
$$

The seven-phase system is well balanced and the first phase is selected for further analysis, as in analytical developments. Different values of $m$ are investigated $\left(1 / 7,2 / 73 / 7\right.$, and $\left.0.513 \approx m_{\max }\right)$, as in Section 3.4 , to cover all the considered cases.

In Figs. 3.27a, 3.28a, 3.29a, and 3.30a the current ripple $\tilde{i}$ obtained in simulations by (3.70) (pink trace) is compared with the peak-to-peak current ripple, $\tilde{i}_{p p}$, evaluated in the different regions by the equations presented in Section 3.4.2 (blue traces), for one fundamental period. Each figure is backed with the enlarged detailed view of the current ripple.

In the corresponding Figs. 3.27b, 3.28b, 3.29b, and 3.30b is depicted the instantaneous output current with the calculated upper/lower ripple envelope, depicted in blue color, for one fundamental period.

The agreement is good in the whole fundamental period. Shown figures (Fig. 3.27 to Fig. 3.30) are for modulation indexes that cover all possible subcases, i.e., all the colored regions in Figure 3.21. This proves the validity of all the equations derived in Section 3.4.2. 
(a)

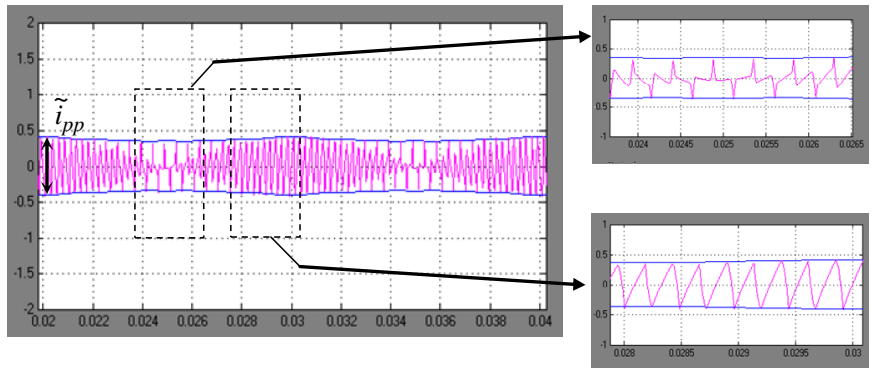

(b)

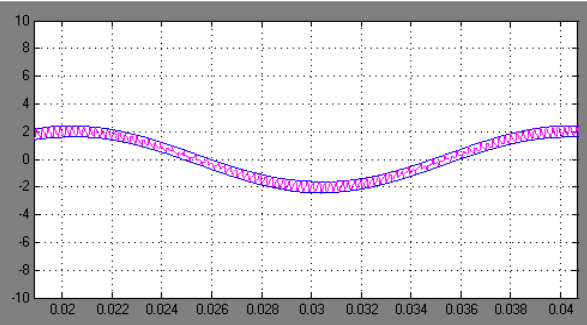

Fig. 3. 27. Simulation results, $m=1 / 7$ : (a) current ripple (pink) and evaluated peak-to-peak envelope (blue), with details; (b) instantaneous output current with calculated envelopes (blue).

(a)

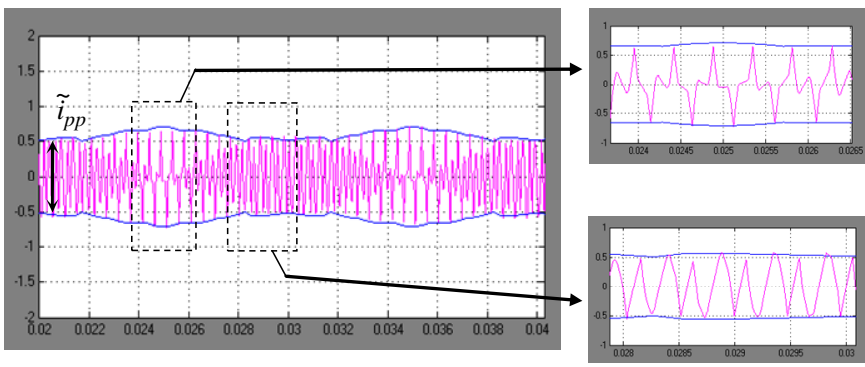

(b)

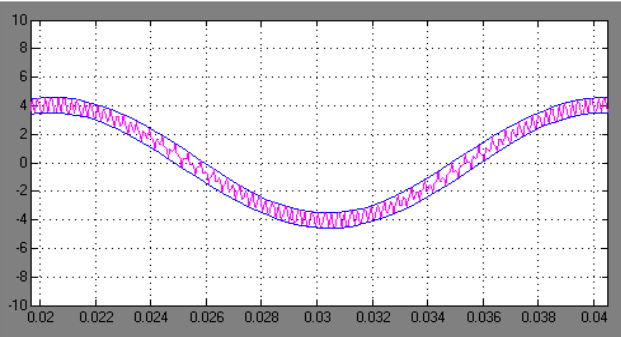

Fig. 3. 28. Simulation results, $m=2 / 7$ : (a) current ripple (pink) and evaluated peak-to-peak envelope (blue), with details; (b) instantaneous output current with calculated envelopes (blue). 
(a)

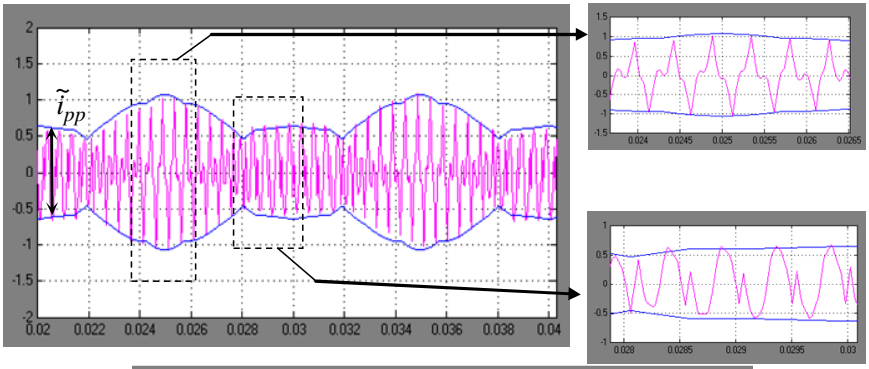

(b)

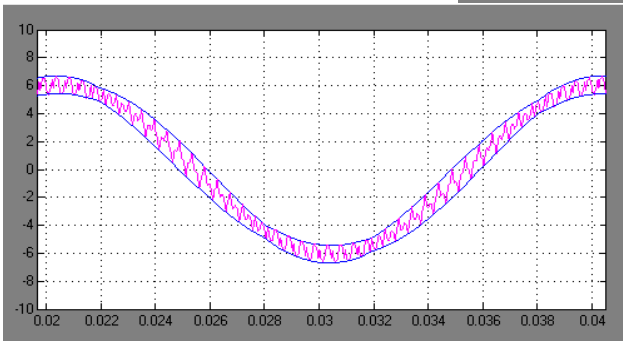

Fig. 3. 29. Simulation results, $m=3 / 7$ : (a) current ripple (pink) and evaluated peak-to-peak envelope (blue), with details; (b) instantaneous output current with calculated envelopes (blue).

(a)

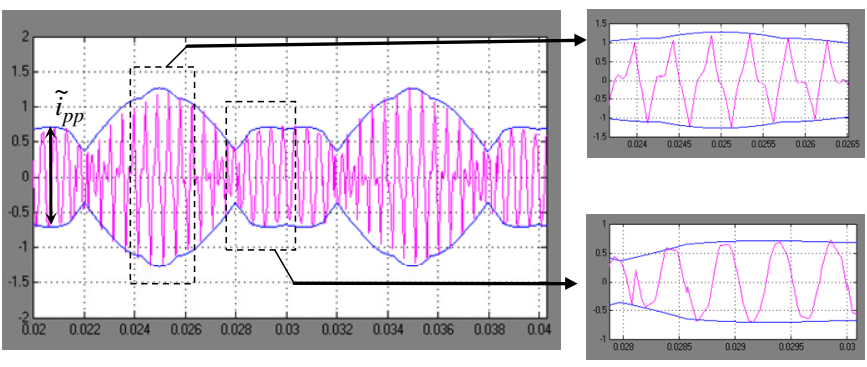

(b)

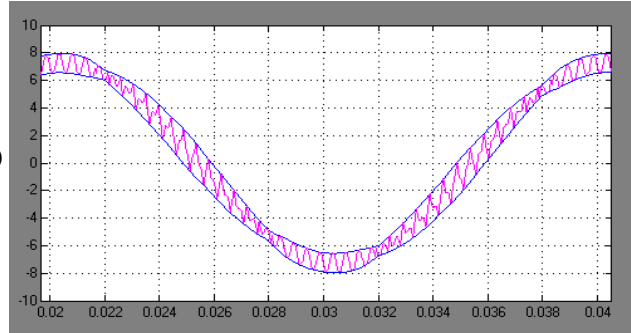

Fig. 3. 30. Simulation results, $m=0.513$ : (a) current ripple (pink) and evaluated peak-to-peak envelope (blue), with details; (b) instantaneous output current with calculated envelopes (blue). 


\subsection{References}

[1] E. Levi, R.Bojoi, F. Profumo, H.A. Toliyat, S. Williamson, "Multi-phase induction motor drives-A technology status review", IET Elect. Power Appl. vol. 1, no. 4, pp. 489-516, July 2007.

[2] H.A. Toliyat, S.P. Waikar, T.A. Lipo, "Analysis and simulation of fivephase synchronous reluctance machines including third harmonic of airgap MMF," IEEE Trans. on Industry Applications, vol. 34, no. 2, pp. 332-339, March/April 1998.

[3] H. Xu, H.A. Toliyat, L.J. Petersen, "Five-phase induction motor drives with DSP-based control system," IEEE Trans. on Power Electronics, vol. 17, no. 4, pp. 524-533, July 2002.

[4] H.M. Ryu, J.W. Kim, S.K. Sul, "Synchronous frame current control of multi-phase synchronous motor. Part I. Modelling and current control based on multiple d-q spaces concept under balanced condition," In Proc. of 39 ${ }^{\text {th }}$ IAS Annual Meeting, 3-7 October 2004, vol. 1, pp. 56-63.

[5] L. Parsa, H.A. Toliyat, "Five-phase permanent-magnet motor drives," IEEE Trans. on Industry Applications, vol. 41, no. 1, pp. 30-37, Jan./Febr. 2005.

[6] E. Levi, D. Dujic, M. Jones, G. Grandi, "Analytical determination of DCbus utilization limits in multi-phase VSI supplied AC drives," IEEE Trans. on Energy Convers, vol. 23, no. 2, pp. 433-443, June 2008.

[7] G. Grandi, G. Serra, A. Tani, "General analysis of multi-phase systems based on space vector approach," In Proc. of $12^{\text {th }}$ Power Electronics and Motion Control Conference (EPE-PEMC), Portoroz SLO, 30 Aug. - 1 Sept. 2006, pp. 834-840.

[8] H.M. Ryu, J.H. Kim, S.K. Sul, "Analysis of multi-phase space vector pulse width modulation based on multiple d-q spaces concept," IEEE Trans. on Power Electronics, vol. 20, no. 6, pp. 1364-1371, Nov. 2005.

[9] A. Iqbal, E. Levi, "Space vector modulation schemes for a five-phase voltage source inverter," In Proc. of European Power Electronic Conference (EPE), Dresden D, 11-14 Sept., 2005, pp. 1-12. 
[10] P.S.N. de Silva, J.E. Fletcher, B.W. Williams, "Development of space vector modulation strategies for five phase voltage source inverters," In Proc. of Power Electronics, Machines and Drives Conference (PEMD), Edinburgh UK, vol. 2, 31 March-2 Apri 2004, pp. 650-655.

[11] O. Ojo, G. Dong, "Generalized discontinuous carrier-based PWM modulation scheme for multi-phase converter-machine systems," In Proc. of $40^{\text {th }}$ Annual Meeting, IEEE Industry Applications Society, Hong Kong, vol. 2, 2-6 Oct. 2005, pp. 1374-1381.

[12] G. Grandi, G. Serra, A. Tani, "Space vector modulation of a seven-phase voltage source inverter," In Proceedings of the International Symposium on Power Electronics, Electrical Drives, Automation and Motion (SPEEDAM), Taormina IT, 23-26 May 2006, pp. 1149-1156.

[13] D. Dujic, E. Levi, G. Serra, A. Tani, L. Zarri, "General modulation strategy for seven-phase inverters with independent control of multiple voltage space vectors," IEEE Trans. Ind. Electron., vol. 55, no. 5, pp. 1921-1932, May 2008.

[14] D. Dujic, E. Levi, M. Jones, G. Grandi, G. Serra, A. Tani, "Continuous PWM techniques for sinusoidal voltage generation with seven-phase voltage source inverters," In Proceedings of the Power Electronics Specialists Conference (IEEE-PESC), Orlando FL, USA, 17-21 June 2007, pp. 47-52.

[15] J.S. Hu, K.Y. Chen, T.Y. Shen, C.H. Tang, "Analytical solutions of multilevel space-vector PWM for multiphase voltage source inverters," IEEE Trans Power Electron., vol. 26, no. 5, pp. 1489-1502, June 2011.

[16] O. Lopez, J. Alvarze, J.D. Gandoy, F.D. Freijedo, "Multiphase space vector PWM algorithm," IEEE Trans Ind. Electron., vol. 55, no. 5, pp. 1933-1942, May 2008.

[17] A. Iqbal and S. Moinuddin, "Comprehensive relationship between carrierbased PWM and space vector PWM in a five-phase VSI,", IEEE Trans. on Power Electronics, vol. 24, no. 10, pp. 2379-2390, Sept. 2009.

[18] D. Casadei, M. Mengoni, G. Serra, A. Tani, L. Zarri "A new carrier-based PWM strategy with minimum output current ripple for five-phase inverters," In Proc. of the $14^{\text {th }}$ European Conference on Power 
Electronics and Applications (EPE), Birmingham UK, 30 Aug.-1 Sept., 2011, pp. 1-10.

[19] D. Dujic, M. Jones, E. Levi, "Analysis of output current ripple rms in multiphase drives using space vector approach," IEEE Trans. on Power Electronics, vol. 24, no. 8, pp. 1926-1938, August 2009.

[20] M. Jones, D. Dujic, E. Levi, J. Prieto, F. Barrero "Switching ripple characteristics of space vector PWM schemes for five-phase two-level voltage source inverters-Part2: Current ripple," IEEE Trans. on Industrial Electronics, vol. 58, no. 7, pp. 2799-2808, July 2011.

[21] P.A. Dahono, Deni, E.G. Supriatna, "Output current-ripple analysis of five-phase PWM inverters," IEEE Trans. on Industry Applications, vol. 45, no. 6, pp. 2022-2029, Nov/Dec 2009.

[22] D. Dujic, M. Jones, E. Levi, "Analysis of output current-ripple RMS in multiphase drives using polygon approach," IEEE Trans. on Power Electronics, vol. 25, no. 7, pp. 1838-1849, July 2010.

[23] D. Jiang, F. Wang, "Current-ripple prediction for three-phase PWM converters," IEEE Trans. on Ind. Applications, vol. 50, no. 1, pp. 531538, Jan/Feb 2014.

[24] D. Jiang, F. Wang, "A general current ripple prediction method for the multiphase voltage source converter," IEEE Trans. on Power Electronics, vol. 29, no. 6, pp. 2643-2648, June 2014.

[25] G. Grandi and J. Loncarski, "Analysis of dead-time effects in multi-phase voltage sourse inverters," In Proc. of IET Power Electronics, Machines and Drives (PEMD), Bristol UK, 27-29 March 2012, CD-ROM paper 0223 .

\subsection{Authored papers}

- G. Grandi, J. Loncarski, "Evaluation of current ripple amplitude in five-phase PWM voltage source inverters," Proc. of IEEE Conference on ICT, Power engineering, and Signal processing (EUROCON), Zagreb CRO, 1-4 July 2013. DOI: $\underline{10.1109 / E U R O C O N .2013 .6625114}$ 
- J. Loncarski, O. Dordevic, G. Grandi, "Experimental verification of current ripple amplitude in five-phase PWM VSIs," In Proc. of $39^{\text {th }}$ Annual Conference of the IEEE Industrial Electronics Society (IECON), Wien AUT, 10-13 Nov. 2013. DOI: 10.1109/IECON.2013.6699977

- G. Grandi, J. Loncarski, “Analysis of peak-to-peak current ripple amplitude in seven-phase PWM voltage source inverters", Energies, 6, ISSN 19961073, 2013. DOI: $\underline{10.3390 / e n 6094429}$ 


\section{Analysis of the output current ripple in multilevel VSIs}

\subsection{Introduction}

Multilevel inverters became more and more popular in last decades, due to the increased power ratings, improved output voltage waveforms, and reduced electromagnetic interference emission. A simple and reliable implementation of multilevel inverter is based on cascaded connection of single-phase inverters (H-bridge), leading to a structure capable of reaching high output voltage amplitudes by using standard low-voltage components. Three-level PWM voltage source inverters (VSIs) are the viable converters for many high-power applications, both grid-connected and motor-load. Compared with traditional two-level inverters, three-level inverters have half of the voltage stress on switching devices for the same maximum output voltage and generate lower harmonics for the same switching frequency.

Nowadays, three commercial topologies of multilevel voltage-source inverters exist: neutral point clamped (NPC), flying capacitors (FCs), and cascaded H-bridge (CHB).

The simplest NPC topology is obtained by doubling the number of switches in comparison to the two-level inverter, and adding the same number of diodes to each additional switch. An additional level is obtained by clamping through the diodes (clamping diodes), that are connected to the neutral point of the source. The output voltage has three states: $V_{d c} / 2,0$, and $-V_{d c} / 2$. The principle of the switching is simple: for $n$ - level inverter highest $(n-1)$ adjacent switches need to be turned on together to achieve maximum leg voltage, next $(n-1)$ switches to be turned on for $(n-2)$-th output level etc. In neutral point clamped inverters, three-level inverter is most popular within this class, because for higher number of level static or stray inductance overvoltage can appear across the switches. In order to solve these problems a different diodeclamped topology has been proposed, which is working without the association of the clamping diodes [1].

The flying capacitor FC topology is derived from diode clamped topology by the elimination of the clamping diodes. FC inverter circuit is with 


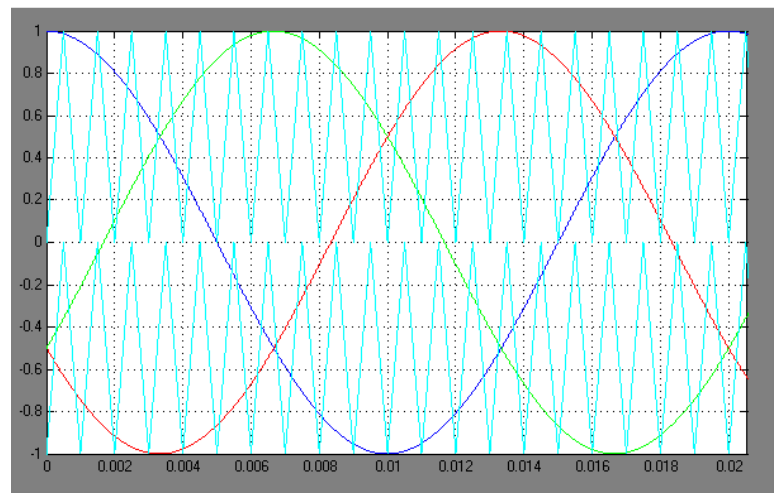

Fig. 4.1. Three-level carrier-based PD modulation (normalized by $V_{d c}$ ), for $m=1$

independent capacitors clamping the device voltage to one capacitor voltage level. The three-level inverter provides output voltage with three states: $V_{d c} / 2$, 0 , and $-V_{d c} / 2$. FC inverter uses additional capacitors oppositely charged to be included in series with dc supply, which have the same role as clamping diodes in diode-clamped inverters. They keep constant the voltage drop between the busses to which they are connected. The charge of can be balanced by proper selection of the 0-level switch combination. Despite that this structure is very similar to the diode-clamped converter, most important difference is that the FC inverter has a modular structure that can be more easily extended to achieve more voltage levels. The main drawbacks of the FC inverters are requirement for significant number of capacitors which are unequally rated, complex control algorithm and many voltage sensors for high number of capacitor voltages to be controlled [2].

Among these inverter topologies, cascaded multilevel inverter reaches the higher output voltage and power levels and the higher reliability due to its modular topology. Cascaded multilevel inverters are based on a series connection of several single-phase inverters. This structure is capable of reaching medium output voltage levels using only standard low-voltage components. These converters also feature a high modularity degree because each inverter can be seen as a module with similar circuit topology, control structure, and modulation. One of the advantages of this type of multilevel inverter is that it needs less number of components compared to the diode- 
clamped or the flying capacitor. In three-level cascaded inverter each singlephase full-bridge inverter generates three output voltage states: $+V_{d c}, 0$, and $V_{d c}$. There are a number of alternative topologies based on the basic principle of cascaded single-phase inverter. Among these, the topology with most interest is modular multilevel converter (MMC), where several dc/dc modules with floating dc link are connected in series to obtain a single-phase or threephase output voltage. The MMC provides high scalability, it has low filter requirements and moreover it does not require an input transformer [3, 4].

\subsubsection{Modulation techniques}

The performance of a three-level inverter depends on its modulation strategy. There have been many three-level PWM techniques developed in last decades [5]-[9]. Generally these techniques can be classified into two categories: carrier-based modulation (CB-PWM), and space vector modulation (SV-PWM).

For CB-PWM each phase reference voltage is compared with two identically shaped but offset triangle carrier waveforms. The frequency of these carriers defines the switching frequency, and their amplitude is the same as dclink voltage, eventually in per units. The most common multilevel techniques with reference to the phase shift of these carriers are: carriers in phase disposition ( $\mathrm{PD}-$ no phase shift between carriers), phase opposition disposition (POD - carriers above the zero line of reference voltage are out of phase with those below this line by 180 degrees), and alternatively in phase opposition disposition (APOD $-180^{\circ}$ phase shift between adjacent carriers). It is generally accepted that the PD strategy creates the lowest line voltage harmonic distortion and the resulting modulation is equivalent to nearest three vectors (NTV). In Fig. 4.1 is shown the example of carrier-based PD modulation technique.

A typical SV-PWM scheme uses the nearest three vectors algorithm to approximate the desired output voltage vector. During each switching period, the triangle in which the reference vector lies is traversed forth and back once. Beginning and ending states of this traverse correspond to the same space vector, which is called pivot vector. These two redundant switching states are called pivot states. In most continuous modulation strategies for a three-level 


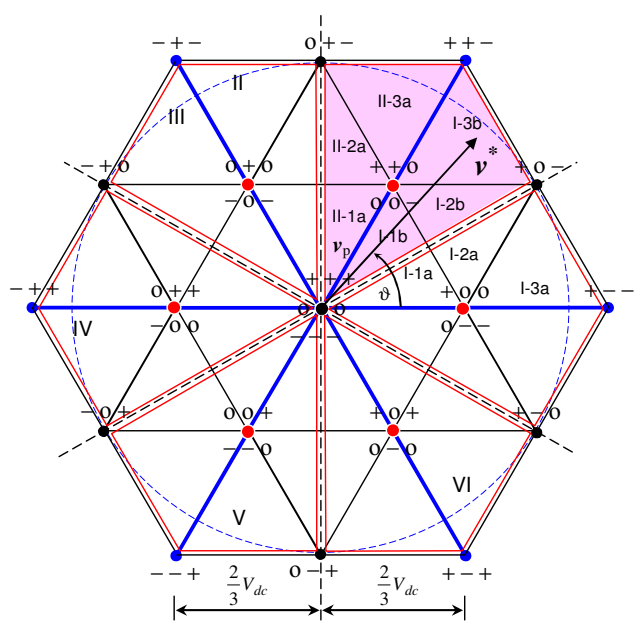

(a)

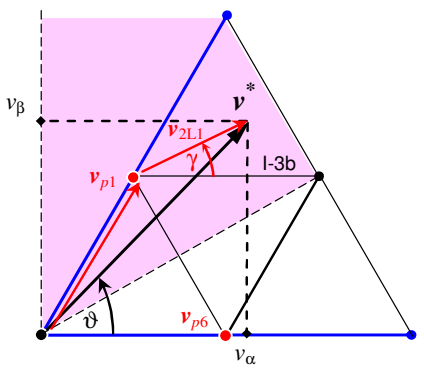

(b)

Fig. 4.2. Space vector diagrams of inverter output voltage: (a) whole hexagon with all six main triangles, (b) detail of first main triangle.

inverter, the switching sequence starts from first pivot state, goes to the second, and symmetrically comes back to the first.

Fig. 4.2(a) shows the output voltage space vectors corresponding to all possible switch configurations. In three-level inverters the redundancy implies availability of multiple switching states to produce a given voltage vector. There is redundancy in zero vector (possible states are $\{+++\},\left\{\begin{array}{lll}0 & 0 & 0\end{array}\right\}$, and $\{---\}$ ), and in all the vectors of magnitude $2 / 3 V_{d c}$ (pivot vectors, red dots in Fig. 4.2) with two possible states. The three nearest voltage vectors are used to synthesize the reference output voltage vector $v^{*}$ in a switching period. For sinusoidal balanced output voltages, the reference output voltage vector is $\boldsymbol{v}^{*}=$ $V^{*} \exp (j \vartheta)$, being $V^{*}=m V_{d c}$ and $\vartheta=\omega t, m$ the modulation index and $V_{d c}$ the dc bus voltage.

The voltage space vector diagram of three-level inverter appears to be a hexagon, consisting of 6 main triangles, numbered I-VI in Fig. 4.2, 12 outer triangles, 6 intermediate, and 6 inner triangles. The hexagon is divided into 6 diamond-shaped sectors, bordered by red lines in Fig. 4.2a. The working domain of each pivot vector is the hexagon centered on it, in this case it is restricted to a diamond-shaped sector (pink colored area in Fig. 4.2), due to the overlaps between sub-hexagons. The switching sequence begins and ends with 
the pivot vector, making the role of pivot vector in three-level VSIs similar to the role of zero vector in case of two-level VSIs.

The multilevel modulation strategy is described with reference to Fig. 4.2b for the first main triangle. The nearest pivot vector $\boldsymbol{v}_{p 1}$ is subtracted from the reference voltage vector $v^{*}$ to obtain the residual reference vector $\boldsymbol{v}_{2 \mathrm{~L} 1}$, that can be synthesized by applying the same procedure as for two-level inverters in the corresponding triangle (I-3b in Fig. 4.2).

It is proved that CB-PWM and SV-PWM strategies for multilevel inverters are equivalent. A proper common-mode injection into the modulating signals of the CB-PWM leads to the same output voltages as in SV-PWM. On the other hand, the CBM can be equivalently realized by SVM through proper sharing of dwell times among the redundant switching states. In particular, the so-called centered PWM (CPWM) obtained by splitting the dwell times of redundant states into equal parts offers lower harmonic distortion in output currents and almost easy implementation with both CBM and SVM techniques [10]- [16].

CB-PWM strategies are widely adopted because of their inherent simplicity and reduced computational requirements compared to SV-PWM, with the possibility of implementation on industrial DSPs without the need additional hardware logics such as FPGA. Furthermore, carrier-based schemes are more flexible since the modulation can be readily handled by a simple commonmode voltage injection, according to

$$
\tilde{v}_{k}^{*}=v_{k}^{*}+v_{c m}, k=\mathrm{a}, \mathrm{b}, \mathrm{c} .
$$

being $v_{k}^{*}$ the reference phase voltages (corresponding to reference space vector $\left.v^{*}\right), v_{c m}$ the injected common-mode voltage, and $\tilde{v}_{k}^{*}$ the resulting modified modulating signals.

There are essentially two different methods available in literature to implement the centered PWM, equivalent to centered space-vector PWM, by the carrier-based modulation. In the first one, the common-mode voltage is determined on the basis of the minimum, middle and maximum values of the reference phase voltages and their correlations [14]. In the second one, the modulus function is introduced for shifting up the reference voltages so their carrier intersections lie within a common carrier band [11], [13]. 


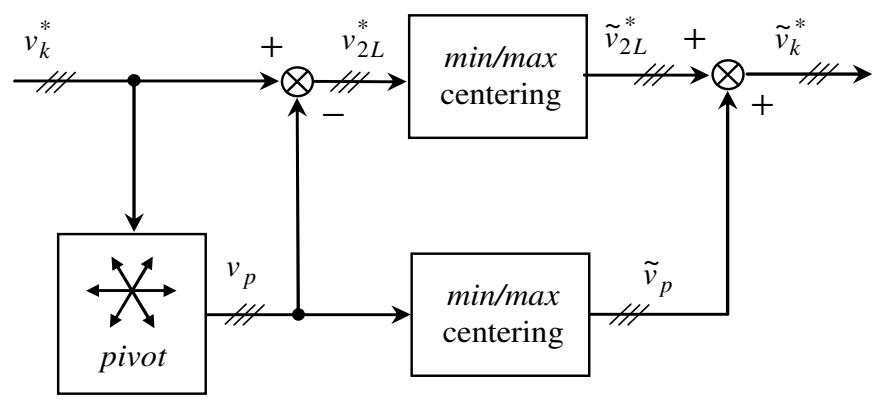

Fig. 4.3. Block diagram of the centered PWM scheme.

A simpler and more comprehensive approach to implement centered PWM is essentially following for the CB-PWM a similar procedure as in SV-PWM. In order to simplify the determination of the common-mode signal to be injected in the reference voltages, a straight and comprehensible method is based on the correspondence between output voltages/space vectors of twolevel and three-level inverters. In particular, taking into account that the pivot vector in three-level inverter has the similar role as the null vector in two-level inverter, a sub-hexagon corresponding to two-level inverter can be indentified within the hexagon of three-level inverter for each pivot vector. The overlaps between sub-hexagons reduce the pivot domain into a diamond-shaped sector (pink colored area in Fig 4.2a for $\boldsymbol{v}_{p 1}$ ). In each pivot sector, the reference voltage $v_{k}{ }^{*}$ of the three-level inverter can be considered as the combination of the pivot voltage $v_{p}$ and the residual two-level reference voltage $v_{2 L}^{*}$, for each phase $k$, as

$$
v_{k}^{*}=v_{p}+v_{2 L}^{*} \text {. }
$$

It is well known that $\min / \max$ common-mode injection realizes centered CB-PWM in two-level inverters. To extend this procedure to three-level inverters, the $\mathrm{min} / \mathrm{max}$ centering is separately applied to the two terms in (4.2), leading to the three-level centered reference voltage $\tilde{v}_{k}^{*}$. This simple method is summarized in the block diagram of Fig. 4.3, leading to the resulting expression

$$
\tilde{v}_{k}^{*}=v_{k}^{*}-\frac{1}{2}\left[\max \left(v_{p}\right)+\min \left(v_{p}\right)\right]-\frac{1}{2}\left[\max \left(v_{2 L}^{*}\right)+\min \left(v_{2 L}^{*}\right)\right] .
$$


The residual two-level reference voltage $v_{2 L}{ }_{2 L}$ is determined by (4.2) and the pivot voltage $v_{p}$ can be readily obtained just on the basis of the sign of reference voltages as

$$
v_{p}=\frac{V_{d c}}{2}\left[\operatorname{sign}\left(v_{k}^{*}\right)-\frac{1}{3} \sum_{k=1}^{3} \operatorname{sign}\left(v_{k}^{*}\right)\right] .
$$

By introducing (4.4) in (4.3) and simplifying leads to

$$
\tilde{v}_{k}^{*}=v_{k}^{*}+\frac{V_{d c}}{6} \sum \operatorname{sign}\left(v_{k}^{*}\right)-\frac{1}{2}\left[\max \left(v_{2 L}^{*}\right)+\min \left(v_{2 L}^{*}\right)\right] .
$$

Fig. 4.4 shows the resulting modified modulating signals $\tilde{v}_{k}^{*}$ (normalized by $V_{d c}$ ) obtained by applying the (4.5) with different modulation indexes, $m=1 / 3$, $2 / 3,1$, and $2 / \sqrt{ } 3=m_{\max }$.

Comparing (4.5) with (4.1) and introducing (4.2), the resulting commonmode signal to be added to the reference voltages is now given by

\section{$m=1 / 3$}

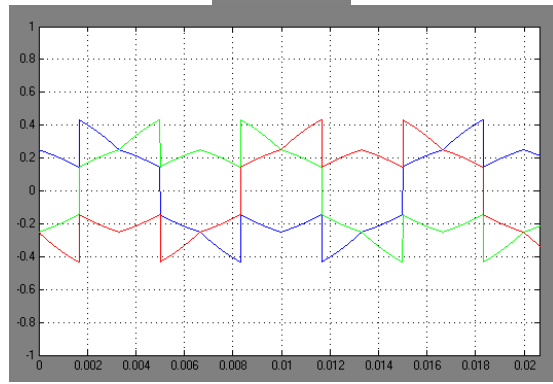

$m=1$

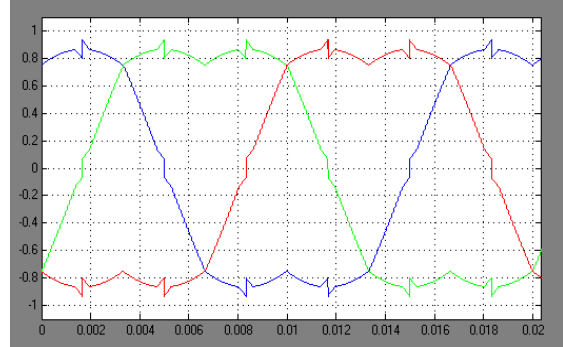

Fig. 4.4. Modified centered modulating signals $\tilde{v}_{k}^{*}$ (normalized by $V_{d c}$ ) for the centered PWM method for different modulation indexes.

\section{$m=2 / 3$}

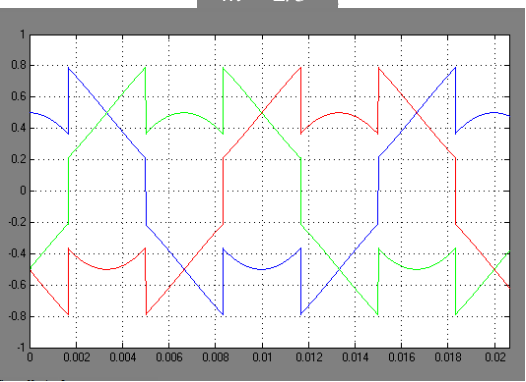

$m=2 / \sqrt{3}$

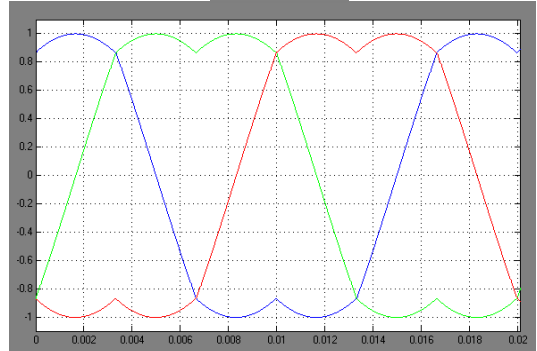




$$
v_{c m}=\frac{V_{d c}}{6} \sum \operatorname{sign}\left(v_{k}^{*}\right)-\frac{1}{2}\left[\max \left(v_{k}^{*}-v_{p}\right)+\min \left(v_{k}^{*}-v_{p}\right)\right] .
$$

The first term in (4.6) is independent by the modulation index, and it is calculated just by the sign of reference voltages, whereas the second term simply consists in the application of the well known min/max centering to the residual two-level voltages. For these reasons $v_{c m}$ can be can be readily synthesized by industrial DSPs, and the implementation of (4.6) with analogical circuitry is quite simple as well.

\subsection{Extension of the peak-to-peak output current ripple analysis to three- level VSIs}

\subsubsection{State of the art}

Various PWM schemes for three-level VSIs with improved output voltage waveforms have been proposed in last decades. In general, output current ripple was studied in terms of the RMS value. In [17] the current ripple trajectory in $\alpha-\beta$ coordinates for the case of dual-inverter-fed open-end winding load configuration is shown, operating as three-level inverter, but emphasis was made to current ripple RMS. The analysis the peak-to-peak output current ripple amplitude in three-phase two-level PWM inverters has been recently introduced in [18]. Similar analysis has been extended to the case of the multiphase inverters [19]. The analysis of the peak-to-peak output current ripple in case of the three-level inverters is missing in the literature.

\subsubsection{Calculation of the peak-to-peak output current ripple}

In the following analysis the centered PWM is considered, as described in Sect. 4.1.1. The reference is made to a generic RLE load, representing both grid-connected and motor loads. The analysis of three-level inverters by the space vector transformation leads to better understanding and more simple calculation of voltage levels and corresponding application times. In the case of a three-level inverter, the output voltage vector can be written similarly to the corresponding two-level inverter, considering the switching states of the $k$ th inverter phase as $S_{k}=[-1,0,1]$ (in the following are summarized as $\{-0+\}$ ): 


$$
\boldsymbol{v}=\frac{2}{3} V_{d c}\left[S_{1}+S_{2} \boldsymbol{\alpha}+S_{3} \boldsymbol{\alpha}^{2}\right] .
$$

being $\boldsymbol{\alpha}=\exp (j 2 \pi / 3)$.

Application times $t_{k}$ are defined by duty-cycles $\delta_{1}, \delta_{2}$, and $\delta_{p}$, and switching period $T_{s}$, being $\delta_{k}=t_{k} / T_{s} / 2$. Duty-cycles are given in Table I for all the triangles named in Fig. 4.2a (first quadrant), by introducing the normalized reference voltages $u_{\alpha}$ and $u_{\beta}$ defined as:

$$
u_{\alpha}=\frac{v_{\alpha}}{V_{d c}}=m \cos \vartheta \text { and } u_{\beta}=\frac{v_{\beta}}{V_{d c}}=m \sin \vartheta,
$$

being $v_{\alpha}$ and $v_{\beta}$ the real and imaginary components of $v^{*}$, as represented in Fig.

Table I: Duty-cycles for triangles in the first quadrant as function of normalized voltages

\begin{tabular}{|l|c|c|c|}
\hline & $\boldsymbol{\delta}_{\boldsymbol{I}}$ & $\boldsymbol{\delta}_{2}$ & $\boldsymbol{\delta}_{p}$ \\
\hline I-1a & $1-\frac{3}{2}\left(u_{\alpha}+1 / \sqrt{3} u_{\beta}\right)$ & $\sqrt{3} u_{\beta}$ & $\frac{3}{2}\left(u_{\alpha}-1 / \sqrt{3} u_{\beta}\right)$ \\
\hline I-1b & $\frac{3}{2}\left(u_{\alpha}-1 / \sqrt{3} u_{\beta}\right)$ & $1-\frac{3}{2}\left(u_{\alpha}+1 / \sqrt{3} u_{\beta}\right)$ & $\sqrt{3} u_{\beta}$ \\
\hline II-1a & $1-\sqrt{3} u_{\beta}$ & $\frac{3}{2}\left(1 / \sqrt{3} u_{\beta}-u_{\alpha}\right)$ & $\frac{3}{2}\left(u_{\alpha}+1 / \sqrt{3} u_{\beta}\right)$ \\
\hline I-2a & $\frac{3}{2}\left(u_{\alpha}+1 / \sqrt{3} u_{\beta}\right)-1$ & $1-\frac{3}{2}\left(u_{\alpha}-1 / \sqrt{3} u_{\beta}\right)$ & $1-\sqrt{3} u_{\beta}$ \\
\hline I-2b & $1-\sqrt{3} u_{\beta}$ & $\frac{3}{2}\left(u_{\alpha}+1 / \sqrt{3} u_{\beta}\right)-1$ & $1-\frac{3}{2}\left(u_{\alpha}-1 / \sqrt{3} u_{\beta}\right)$ \\
\hline II-2a & $1-\frac{3}{2}\left(u_{\alpha}+1 / \sqrt{3} u_{\beta}\right)$ & $\sqrt{3} u_{\beta}-1$ & $1-\frac{3}{2}\left(1 / \sqrt{3} u_{\beta}-u_{\alpha}\right)$ \\
\hline I-3a & $\sqrt{3} u_{\beta}$ & $\frac{3}{2}\left(u_{\alpha}-1 / \sqrt{3} u_{\beta}\right)-1$ & $2-\frac{3}{2}\left(u_{\alpha}+1 / \sqrt{3} u_{\beta}\right)$ \\
\hline I-3b & $\sqrt{3} u_{\beta}-1$ & $\frac{3}{2}\left(u_{\alpha}-1 / \sqrt{3} u_{\beta}\right)$ & $2-\frac{3}{2}\left(u_{\alpha}+1 / \sqrt{3} u_{\beta}\right)$ \\
\hline II-3a & $\frac{3}{2}\left(1 / \sqrt{3} u_{\beta}-u_{\alpha}\right)$ & $\frac{3}{2}\left(u_{\alpha}+1 / \sqrt{3} u_{\beta}\right)-1$ & $2-\sqrt{3} u_{\beta}$ \\
\hline
\end{tabular}


4.2b. The modulation limit is $m \leq m_{\max }=2 / \sqrt{ } 3 \approx 1.155$.

In the considered case of sinusoidal balanced currents, the three-phase system is symmetric and only one phase can be analyzed. Considering the first phase, the variables are given by extracting the real part of the corresponding space vectors, which are their projection on the real axes. If the reference voltage is within the modulation limits, i.e., the reference space vector $v^{*}$ lies within the outer hexagon, the average inverter output voltage (first phase) is given by

$$
\bar{v}\left(T_{s}\right)=v^{*}=\operatorname{Re}\left\{v^{*}\right\}=V^{*} \cos \vartheta=V_{d c} m \cos \vartheta
$$

The instantaneous output phase voltage $v(t)$ can be expressed by the switching states. For the first phase it can be calculated as the real part of the output voltage vector (4.7), leading to

$$
v=V_{d c}\left[S_{1}-\frac{1}{3}\left(S_{1}+S_{2}+S_{3}\right)\right] .
$$

By introducing (4.9) and (4.10) in the expression of the alternating voltage defined in Chapter 2, (2.6), the alternating output voltage component is

$$
\tilde{v}(t)=V_{d c}\left[S_{1}-\frac{1}{3}\left(S_{1}+S_{2}+S_{3}\right)\right]-V_{d c} m \cos \vartheta .
$$

The ripple evaluation in three-level VSIs can be obtained in two different ways: (A) basing the ripple evaluation on the developments carried out for two-level VSIs, introducing the concept of pivot voltage vector instead of null voltage vector, and (B) directly by analyzing the output voltage waveforms of the three-level inverter, as done for two-level inverters in previous chapters. Both ways to obtain the current ripple will be presented in the following. 


\section{A) Ripple evaluation by introducing pivot vector and two-level analysis}

Considering the quarter wave symmetry, the evaluation of the current ripple can be restricted to the phase angle range $\left[0-90^{\circ}\right]$. In Fig. 4.5 is represented the $1^{\text {st }}$ quadrant of the output voltage hexagon for two-and three-level inverter. Space vector hexagon of two-level inverter can be identified within the space vector hexagon of three-level inverter, as pointed out for the first quadrant in Fig. 4.5. Different colors represent the different cases, first quadrant of twolevel inverter (sector I and half of the sector II) and the corresponding quadrant of three-level inverter (triangle I-3b and half of triangle II-3a), and should be considered separately for the ripple evaluation. For the same dc-bus voltage, the first quadrant of the two-level inverter is the same as the corresponding quadrant in three-level inverter, just shifted for the pivot vector $\boldsymbol{v}_{p 1}$. Taking this into account, the current ripple of the three-level inverter can be determined in easy way, by starting from the evaluation for two-level inverter in the three identified cases.

In Fig. 4.6 is depicted the current ripple $\tilde{i}$ and its peak-to-peak value $\tilde{i}_{p p}$ together with the instantaneous output voltage $v(t)$ for two-level inverter and for each colored triangle indentified in Fig. 4.5, in a separate diagram. The switching sequence is chosen in such way to correspond to the switching sequence of three-level inverter for a specific case. Duty-cycles defined as $\delta_{k}=$ $t_{k} / T_{s} / 2$, are given in Table II for all the colored regions of Fig. 4.5, by introducing the normalized reference voltages $u_{\alpha}$ and $u_{\beta}$ defined by (4.8).

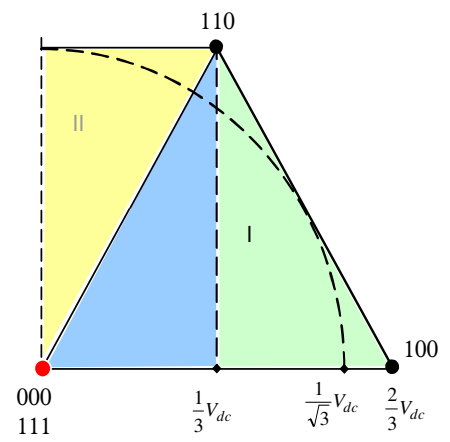

two-level inverter

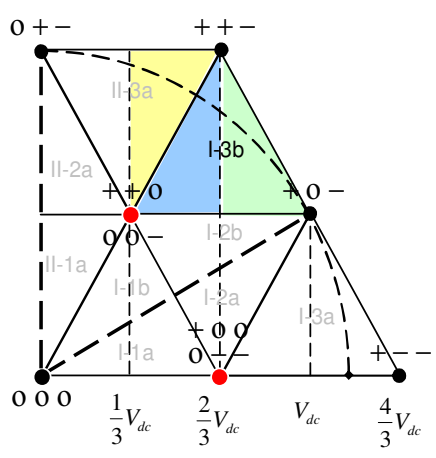

three-level inverter

Fig. 4.5. Output voltage diagram of two-level and three-level inverter in the first quadrant of $\alpha-\beta$ plane. 
Table II: Application duty-cycles of two-level inverter in considered cases

\begin{tabular}{|c|c|c|c|c|c|c|}
\hline & $\delta_{1}$ & $S_{1} S_{2} S_{3}$ & $\delta_{2}$ & $S_{1} S_{2} S_{3}$ & $\delta_{0}$ & $S_{1} S_{2} S_{3}$ \\
\hline I & $\sqrt{3} u_{\beta}$ & 110 & $\frac{3}{2}\left(u_{\alpha}-1 / \sqrt{3} u_{\beta}\right)$ & 100 & $1-\frac{3}{2}\left(u_{\alpha}+1 / \sqrt{3} u_{\beta}\right)$ & $\begin{array}{lll}1 & 1 & 1 \\
0 & 0 & 0\end{array}$ \\
\hline II & $\frac{3}{2}\left(1 / \sqrt{3} u_{\beta}-u_{\alpha}\right)$ & 010 & $\frac{3}{2}\left(u_{\alpha}+1 / \sqrt{3} u_{\beta}\right)$ & 110 & $1-\sqrt{3} u_{\beta}$ & $\begin{array}{lll}0 & 0 & 0 \\
1 & 1 & 1\end{array}$ \\
\hline
\end{tabular}

By comparing the first quadrant of the two-level inverter with the corresponding quadrant in three-level inverter in Fig. 4.5, it can be noted that the quadrant of three-level inverter is shifted for the pivot vector $\boldsymbol{v}_{p 1}=1 / 3+j$ $1 / \sqrt{ } 3$. According to this, the new normalized reference voltages in the case of three-level inverter are

$$
\begin{aligned}
& u_{\alpha}^{\prime}=u_{\alpha}+1 / 3, \\
& u_{\beta}^{\prime}=u_{\beta}+1 / \sqrt{3},
\end{aligned}
$$

where $u_{\alpha}$ and $u_{\beta}$ are the reference voltages of the two-level inverter. The new reference voltages, $u_{\alpha}$ and $u_{\beta}$, that need to be considered in the expressions for

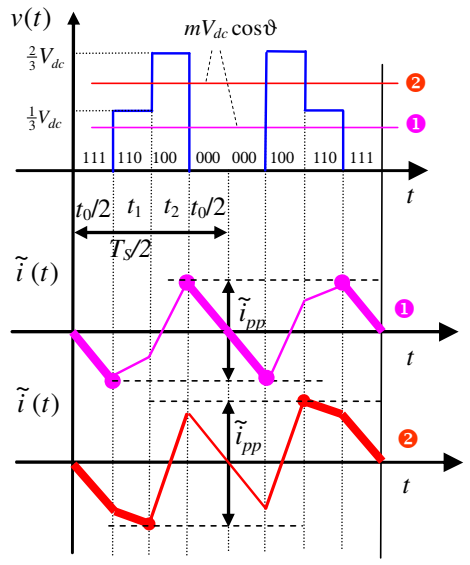

Sector I

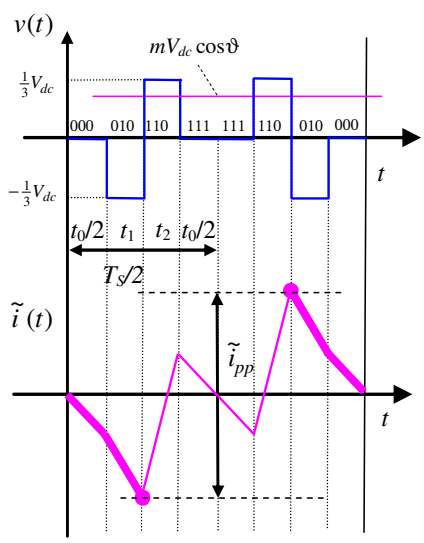

Sector II

Fig. 4.6. Output voltage and current ripple in one switching period for two-level inverter. 
the ripple evaluation of two-level inverter to obtain the ripple in three-level inverter are

$$
\begin{gathered}
u_{\alpha}=u_{\alpha}^{\prime}-1 / 3, \\
u_{\beta}=u_{\beta}^{\prime}-1 / \sqrt{3} .
\end{gathered}
$$

The sub-case $m \cos \vartheta \leq 2 / 3$, representing the blue area of triangle I-3b in Fig. 4.5 corresponds to the blue area of sector $\mathrm{I}$ in two-level inverter. Considering the blue area of sector I in two-level inverter, $\tilde{i}_{p p}$ can be evaluated by the expressions for the instantaneous current ripple and its peakto-peak value, (2.10) and (2.11), and (4.11), with application duty-cycle $\delta_{0}$ leading to

$$
\tilde{i}_{p p}=\frac{V_{d c} T_{s}}{2 L}\left\{u_{\alpha} \delta_{0}\right\} .
$$

By substituting the new reference voltages (4.14) and (4.15) in (4.16), $\tilde{i}_{p p}$ in three-level inverter can be determined as

$$
\tilde{i}_{p p}=\frac{V_{d c}}{2} \frac{T_{s}}{L}\left[\left(u_{\alpha}^{\prime}-\frac{1}{3}\right) \delta_{0}^{\prime}\right]
$$

being $\delta_{0}^{\prime}$ the duty-cycle of three-level inverter, which can be obtained starting from the duty-cycle of two-level inverter from Table II and introducing the reference voltages (4.14) and (4.15).

Peak-to-peak current ripple can also be expressed as

$$
\tilde{i}_{p p}=\frac{V_{d c} T_{s}}{2 L} r(m, \vartheta),
$$

being $r(m, \vartheta)$ the normalized peak-to-peak current ripple amplitude.

Finally, after normalizing (4.18), for the normalized current ripple of threelevel inverter can be written

$$
\begin{gathered}
r(m, \vartheta)=\left(u_{\alpha}^{\prime}-\frac{1}{3}\right)\left[1-\frac{3}{2}\left(\left(u_{\alpha}^{\prime}-\frac{1}{3}\right)+\frac{1}{\sqrt{3}}\left(u_{\beta}^{\prime}-\frac{1}{\sqrt{3}}\right)\right)\right]= \\
=\left(u_{\alpha}^{\prime}-\frac{1}{3}\right)\left[2-\frac{3}{2}\left(u_{\alpha}^{\prime}+\frac{1}{\sqrt{3}} u_{\beta}^{\prime}\right)\right]
\end{gathered}
$$


The sub-case $m \cos \vartheta>2 / 3$, representing the green area of triangle I-3b in Fig. 4.5, corresponds to the green area of sector I in two-level inverter. Considering the green area of sector I in two-level inverter, $\tilde{i}_{p p}$ can be evaluated by (2.10), (2.11), and (4.11), with application duty-cycles $\delta_{0} / 2$ and $\delta_{1}$ leading to

$$
\tilde{i}_{p p}=\frac{V_{d c} T_{s}}{2 L}\left\{u_{\alpha} \delta_{0}+2\left(u_{\alpha}-\frac{1}{3}\right) \delta_{1}\right\} .
$$

By substituting the new reference voltages (4.14) and (4.15) in (4.20), $\widetilde{i}_{p p}$ in three-level inverter can be determined as

$$
\tilde{i}_{p p}=\frac{V_{d c} T_{s}}{2 L}\left\{\left(u_{\alpha}^{\prime}-\frac{1}{3}\right) \delta_{0}^{\prime}+2\left[\left(u_{\alpha}^{\prime}-\frac{1}{3}\right)-\frac{1}{3}\right] \delta_{1}^{\prime}\right\} .
$$

being $\delta_{0}^{\prime}$ and $\delta_{1}^{\prime}$ the duty-cycles of three-level inverter, which can be obtained starting from the duty-cycles of two-level inverter given in Table II, and by introducing the normalized voltages (4.14) and (4.15). Finally, after normalizing (4.18), for the normalized current ripple of three-level inverter can be written

$$
\begin{aligned}
r(m, \vartheta) & =\left(u_{\alpha}^{\prime}-\frac{1}{3}\right)\left\{1-\frac{3}{2}\left[\left(u_{\alpha}^{\prime}-\frac{1}{3}\right)+\frac{1}{\sqrt{3}}\left(u_{\beta}^{\prime}-\frac{1}{\sqrt{3}}\right)\right]\right\}+\left(u_{\alpha}^{\prime}-\frac{2}{3}\right) 2 \sqrt{3}\left(u_{\beta}^{\prime}-\frac{1}{\sqrt{3}}\right)= \\
& =\left(u_{\alpha}^{\prime}-\frac{1}{3}\right)\left[2-\frac{3}{2}\left(u_{\alpha}^{\prime}+\frac{1}{\sqrt{3}} u_{\beta}^{\prime}\right)\right]+2\left(u_{\alpha}^{\prime}-\frac{2}{3}\right)\left(\sqrt{3} u_{\beta}^{\prime}-1\right)
\end{aligned}
$$

The sub-case representing the yellow area of the half of triangle II-3a in Fig. 4.5 , corresponds to the yellow area of the half of sector II in two-level inverter. Considering the yellow area of sector II in two-level inverter, $\tilde{i}_{p p}$ can be evaluated by (2.10), (2.11), and (4.11), with application duty-cycles $\delta_{0}$ and $\delta_{1}$ leading to

$$
\tilde{i}_{p p}=\frac{V_{d c} T_{s}}{2 L}\left\{u_{\alpha} \delta_{0}+2\left(u_{\alpha}+\frac{1}{3}\right) \delta_{1}\right\} .
$$

By substituting the reference voltages (4.14) and (4.15) in (4.23), $\tilde{i}_{p p}$ in three-level inverter can be determined as 


$$
\tilde{i}_{p p}=\frac{V_{d c} T_{s}}{2 L}\left\{\left(u_{\alpha}^{\prime}-\frac{1}{3}\right) \delta_{0}^{\prime}+2\left[\left(u_{\alpha}^{\prime}-\frac{1}{3}\right)+\frac{1}{3}\right] \delta_{1}^{\prime}\right\},
$$

where $\delta_{0}^{\prime}$ and $\delta_{1}^{\prime}$ are the duty-cycles of three-level inverter, which can be obtained starting from the duty-cycles of two-level inverter for sector II, given in Table II, and introducing the equations (4.14) and (4.15). Finally, after normalizing (4.18), for the normalized current ripple of three-level inverter can be written:

$$
\begin{gathered}
r(m, \vartheta)=\left(u_{\alpha}^{\prime}-\frac{1}{3}\right)\left[1-\sqrt{3}\left(u_{\beta}^{\prime}-\frac{1}{\sqrt{3}}\right)\right]+2 u_{\alpha}^{\prime} \frac{3}{2}\left[\frac{1}{\sqrt{3}}\left(u_{\beta}^{\prime}-\frac{1}{\sqrt{3}}\right)-\left(u_{\alpha}^{\prime}-\frac{1}{3}\right)\right]= \\
=\left(u_{\alpha}^{\prime}-\frac{1}{3}\right)\left(2-\sqrt{3} u_{\beta}^{\prime}\right)+3 u_{\alpha}^{\prime}\left(\frac{1}{\sqrt{3}} u_{\beta}^{\prime}-u_{\alpha}^{\prime}\right) .
\end{gathered}
$$

The current ripple evaluated in this way is representing just the part of the whole $1^{\text {st }}$ quadrant of the three-level inverter. The ripple analysis needs to be extended to the whole $1^{\text {st }}$ pivot sector and also to the part of the $2^{\text {nd }}$ pivot sector, according to Fig. 4.5. The ripple evaluation in the remaining regions of pivot sector $\boldsymbol{v}_{p 1}$ can be carried out by exploiting the quarter-wave symmetry of the two-level inverter. The same procedure can be adopted to determine the current ripple in the other pivot sector $\boldsymbol{v}_{p 6}$, to complete the analysis of the $1^{\text {st }}$ quadrant.

\section{B) Ripple evaluation by analyzing the three-level voltage waveforms}

The other method for obtaining the peak-to-peak amplitude of the output current ripple in three-level inverter is based directly on analyzing the output voltage waveforms, following the same procedure given in previous chapters for two-level inverters. Considering the quarter wave symmetry, the current ripple evaluation in the first quadrant, $0<\vartheta<\pi / 2$, can be carried out by considering the three main sectors $0 \leq \vartheta \leq \pi / 6, \pi / 6 \leq \vartheta \leq \pi / 3$, and $\pi / 3 \leq \vartheta \leq$ $\pi / 2$, taking into account all the sub-cases matching the colored areas represented in Fig. 4.7, as described in the following.

The alternating voltage and the current ripple are summarized in the separate diagrams for inner, intermediate, and outer triangles, from Fig. 4.8 to Fig. 4.10, respectively. In all cases, two different current ripple peaks are 
observed in the switching period. Due to the modulation symmetry, positive and negative peaks are matching. In general, for each sector and for the different ranges of $m \cos \vartheta$, one peak results always to be equal or bigger than the other, by observing both current slopes and the corresponding application times.

\section{1) Evaluation in the first sector}

In the first sector, $0 \leq \vartheta \leq \pi / 6$, five different sub-cases are identified, related to the different colored regions in Fig. 4.7.

Instantaneous output voltage $v(t)$, current ripple $\tilde{i}$ and peak-to-peak value $\tilde{i}_{p p}$ for the sub-case $0 \leq m \cos \vartheta \leq 1 / 3$, are presented in diagram 1 of Fig. 4.8a, corresponding to the yellow area of the triangle I-1a in Fig. 4.7. Introducing the expressions for the instantaneous current ripple and its peak-to-peak value, (2.10) and (2.11), and taking into account of (4.11), the switch configurations $\{\mathrm{o}--\}$ and $\left\{\begin{array}{ll}\mathrm{o} & \mathrm{o}-\}\end{array}\right\}$, with the corresponding duty-cycles $\delta_{\mathrm{p}} / 2$ and $\delta_{2}$, can be considered to evaluate $\tilde{i}_{p p}$, according to Fig. $4.8 \mathrm{a}$, leading to

$$
\tilde{i}_{p p}=\frac{V_{d c} T_{s}}{L}\left\{\left(\frac{2}{3}-m \cos \vartheta\right) \frac{\delta_{p}}{2}+\left(\frac{1}{3}-m \cos \vartheta\right) \delta_{2}\right\} .
$$

On the basis of the duty-cycles given in Table I, and introducing $u_{\alpha}$, and

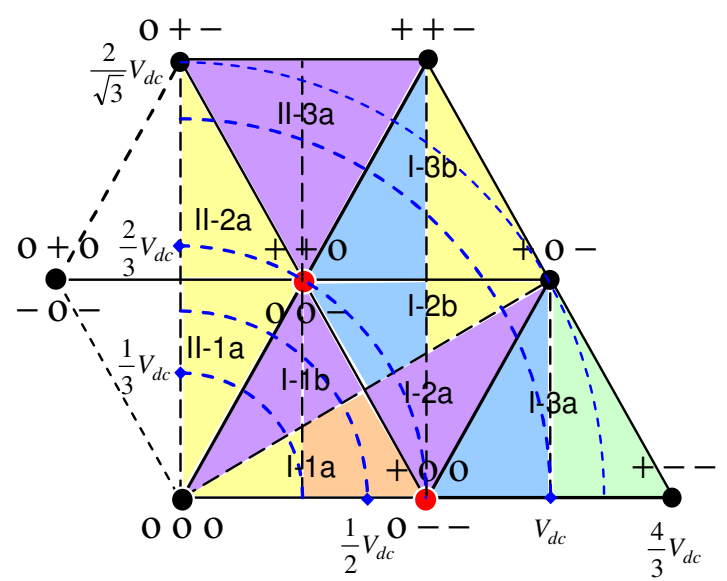

Fig. 4.7. Space vector diagram of inverter output voltage with areas identified for ripple evaluation in the first quadrant, blue dashed circles represent the cases with $m=1 / 3,1 / 2,2 / 3,1,2 / \sqrt{ } 3\left(m_{\max }\right)$. 
$u_{\beta},(4.8)$, the normalized current ripple $r(m, \vartheta)$ defined by $(4.18)$ for this subcase of triangle I-1a becomes

$$
\begin{aligned}
r(m, \vartheta) & =\left(\frac{2}{3}-m \cos \vartheta\right) \delta_{p}+2\left(\frac{1}{3}-m \cos \vartheta\right) \delta_{2}= \\
= & \frac{3}{2}\left(\frac{2}{3}-u_{\alpha}\right)\left(u_{\alpha}-\frac{1}{\sqrt{3}} u_{\beta}\right)+2\left(\frac{1}{3}-u_{\alpha}\right) \sqrt{3} u_{\beta} .
\end{aligned}
$$

The sub-case $m \cos \vartheta>1 / 3$, corresponding to the orange region of triangle I1a in Fig. 4.7, is depicted in diagram 2 of Fig. 4.8a. In this case $\tilde{i}_{p p}$ can be evaluated considering the switch configuration $\{\mathrm{O}--\}$, with the corresponding duty- cycle $\delta_{p} / 2$, leading to

$$
\tilde{i}_{p p}=\frac{V_{d c} T_{s}}{L}\left\{\left(\frac{2}{3}-m \cos \vartheta\right) \frac{\delta_{p}}{2}\right\} .
$$

Introducing the expression for $\delta_{p}$ given in Table I and normalizing, the current ripple becomes

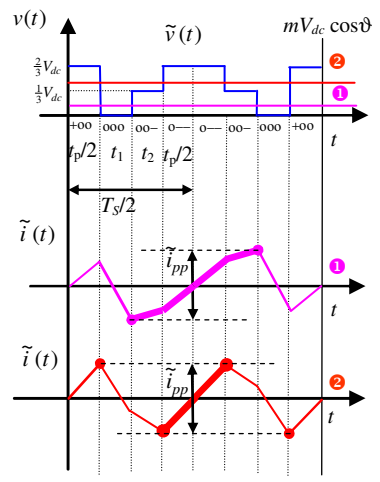

(a) triangle I-1a

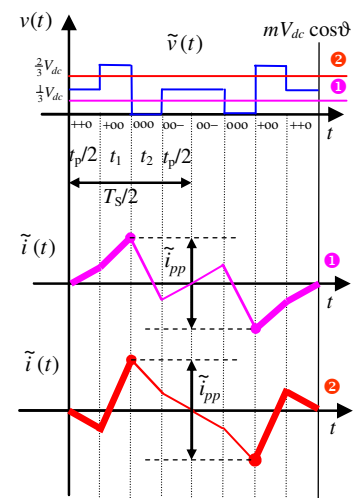

(b) triangle $\mathrm{I}-1 \mathrm{~b}$

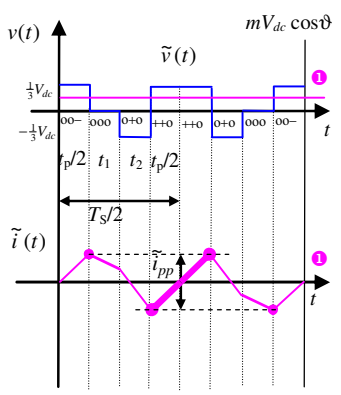

(c) triangle II- $1 \mathrm{a}$

Fig. 4.8. Inner triangles: instantaneous output voltage $v(t)$, current ripple $\tilde{i}$ and peak-to-peak value $\tilde{i}_{p p}$. 


$$
r(m, \vartheta)=\frac{3}{2}\left(\frac{2}{3}-u_{\alpha}\right)\left(u_{\alpha}-\frac{1}{\sqrt{3}} u_{\beta}\right) .
$$

The sub-cases $m \cos \vartheta \leq 2 / 3$ and $m \cos \vartheta>2 / 3$, both corresponding to the purple triangle I-2a in Fig. 4.7, are depicted in diagrams $(1$ and 2 of Fig. 4.9a. In both cases the switch configurations $\{+0 \mathrm{o}\}$ and $\{+0-\}$, with the corresponding duty-cycles $\delta_{p} / 2$ and $\delta_{1}$, can be considered to evaluate $\tilde{i}_{p p}$, leading to

$$
\tilde{i}_{p p}=\frac{V_{d c} T_{s}}{L}\left\{\left(\frac{2}{3}-m \cos \vartheta\right) \frac{\delta_{p}}{2}+(1-m \cos \vartheta) \delta_{1}\right\} .
$$

Introducing the expression for $\delta_{p}$ and $\delta_{1}$ given in Table I and normalizing, the current ripple becomes

$$
r(m, \vartheta)=\left(\frac{2}{3}-u_{\alpha}\right)\left(1-\sqrt{3} u_{\beta}\right)+2\left(1-u_{\alpha}\right)\left[\frac{3}{2}\left(u_{\alpha}+\frac{1}{\sqrt{3}} u_{\beta}\right)-1\right] .
$$

The sub-case $2 / 3 \leq m \cos \vartheta \leq 1$, corresponding to the blue area of triangle I$3 \mathrm{a}$ in Fig. 4.7, is presented in diagram 1 of Fig. 4.10a. In this case, the switch configuration $\{\mathrm{o}--\}$ with the corresponding duty-cycle $\delta_{p} / 2$ can be

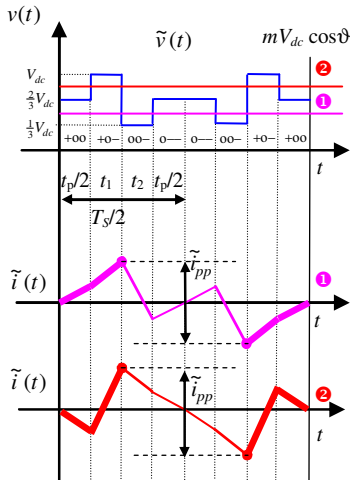

(a) triangle I-2a

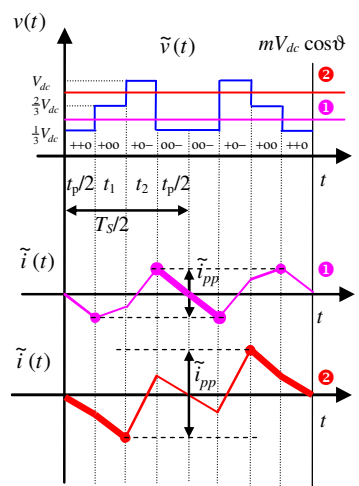

(b) triangle I-2b

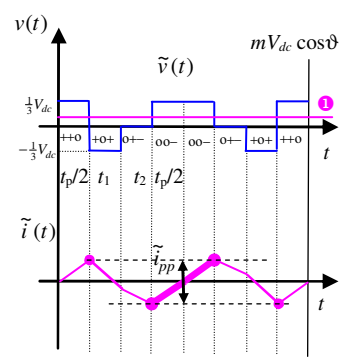

(c) triangle II-2a

Fig. 4.9. Intermediate triangles: instantaneous output voltage $v(t)$, current ripple $\tilde{i}$ and peak-topeak value $\tilde{i}_{p p}$. 
considered to evaluate $\tilde{i}_{p p}$, leading to

$$
\tilde{i}_{p p}=\frac{V_{d c} T_{s}}{L}\left\{\left(m \cos \vartheta-\frac{2}{3}\right) \frac{\delta_{p}}{2}\right\} .
$$

Introducing the expression for $\delta_{p}$ given in Table I and normalizing, the current ripple becomes

$$
r(m, \vartheta)=\left(u_{\alpha}-\frac{2}{3}\right)\left[2-\frac{3}{2}\left(u_{\alpha}+\frac{1}{\sqrt{3}} u_{\beta}\right)\right] .
$$

The sub-case $1 \leq m \cos \vartheta \leq m_{\max } \cos \vartheta<4 / 3$, corresponding to the green area of triangle I-3a in Fig. 4.7, is presented in diagram 2 of Fig. 4.10a. In this case the switch configurations $\{+0 \mathrm{O}\}$ and $\{+\mathrm{O}-\}$, with the corresponding dutycycles $\delta_{p} / 2$, and $\delta_{1}$, can be considered to evaluate $\tilde{i}_{p p}$, leading to

$$
\tilde{i}_{p p}=\frac{V_{d c} T_{s}}{L}\left\{\left(m \cos \vartheta-\frac{2}{3}\right) \frac{\delta_{p}}{2}+(m \cos \vartheta-1) \delta_{1}\right\} .
$$

Introducing the expression for $\delta_{p}$ and $\delta_{1}$ given in Table I and normalizing, the current ripple becomes

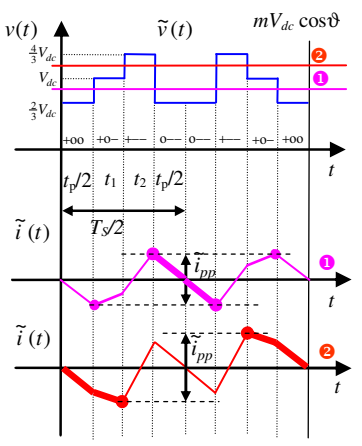

(a) triangle I-3a

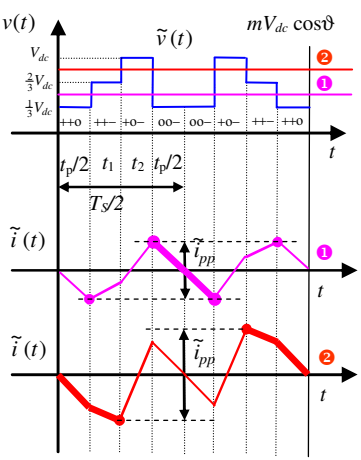

(b) triangle I-3b

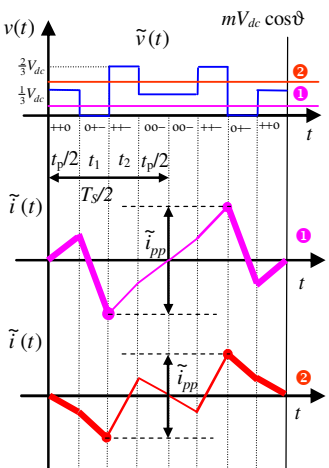

(c) triangle II- $3 \mathrm{a}$

Fig. 4.10. Outer triangles: instantaneous output voltage $v(t)$, current ripple $\tilde{i}$ and peak-to-peak value $\tilde{i}_{p p}$. 


$$
r(m, \vartheta)=\left(u_{\alpha}-\frac{2}{3}\right)\left[2-\frac{3}{2}\left(u_{\alpha}+\frac{1}{\sqrt{3}} u_{\beta}\right)\right]+2\left(u_{\alpha}-1\right) \sqrt{3} u_{\beta} .
$$

\section{2) Evaluation in the second sector}

In the second sector, $\pi / 6 \leq \vartheta \leq \pi / 3$, three different sub-cases are identified, related to the three colored regions in Fig. 4.7.

The sub-cases $0 \leq m \cos \vartheta \leq 1 / 3$ and $m \cos \vartheta>1 / 3$, both corresponding to the purple triangle I-1b in Fig. 4.7, are presented in diagrams (1) and 2 of Fig. 4.8b. According to this figure, the switch configurations $\{++0\}$ and $\{+0 \mathrm{o}\}$, with the corresponding duty-cycles $\delta_{p} / 2$ and $\delta_{1}$, can be considered to evaluate $\tilde{i}_{p p}$ for both sub-cases, leading to

$$
\tilde{i}_{p p}=\frac{V_{d c} T_{s}}{L}\left\{\left(\frac{1}{3}-m \cos \vartheta\right) \frac{\delta_{p}}{2}+\left(\frac{2}{3}-m \cos \vartheta\right) \delta_{1}\right\} .
$$

Introducing the expressions for $\delta_{p}$ and $\delta_{1}$ given in Table I and normalizing by (4.8) and (4.16), the current ripple becomes

$$
r(m, \vartheta)=\left(\frac{1}{3}-u_{\alpha}\right) \sqrt{3} u_{\beta}+3\left(\frac{2}{3}-u_{\alpha}\right)\left(u_{\alpha}-\frac{1}{\sqrt{3}} u_{\beta}\right) .
$$

The sub-cases $m \cos \vartheta \leq 2 / 3$, corresponding to the blue area of triangles I-2b and I-3b in Fig. 4.7, are presented in diagram $\mathbf{0}$ of Fig. 4.9b and in diagram (1) of Fig. 4.10b. For both sub-cases the switch configuration $\{\mathrm{o} \mathrm{O}-\}^{-}$with the corresponding duty-cycle $\delta_{p} / 2$, can be considered to evaluate $\tilde{i}_{p p}$, leading to

$$
\tilde{i}_{p p}=\frac{V_{d c} T_{s}}{L}\left\{\left(m \cos \vartheta-\frac{1}{3}\right) \frac{\delta_{p}}{2}\right\} .
$$

Introducing the expressions for $\delta_{p}$ given in Table I and normalizing, the current ripple becomes

$$
r(m, \vartheta)=\left(u_{\alpha}-\frac{1}{3}\right)\left[1-\frac{3}{2}\left(u_{\alpha}-\frac{1}{\sqrt{3}} u_{\beta}\right)\right], \text { for triangle I- } 2 \mathrm{~b},
$$




$$
r(m, \vartheta)=\left(u_{\alpha}-\frac{1}{3}\right)\left[2-\frac{3}{2}\left(u_{\alpha}+\frac{1}{\sqrt{3}} u_{\beta}\right)\right], \text { for triangle I- } 3 \mathrm{~b} .
$$

The obtained equation for the triangle 1-3b (blue area) can be compared to the corresponding equation (4.19) obtained by the first method (A) of the current ripple evaluation, and obviously it is matching.

The sub-cases $m \cos \vartheta>2 / 3$, corresponding to the yellow area of triangles I$2 \mathrm{~b}$ and I-3b in Fig. 4.7, are presented in diagram 2 of Fig. $4.9 \mathrm{~b}$ and in diagram 2 of Fig. 4.10b. In these sub-cases the switch configurations $\{++0\}$ and $\{+o \quad 0\}$ for triangle $\mathrm{I}-2 \mathrm{~b}$, and $\{++\mathrm{o}\}$ and $\{++-\}$ for triangle I-3b, with the corresponding duty-cycles $\delta_{p} / 2$ and $\delta_{1}$ for both, can be considered to evaluate $\tilde{i}_{p p}$, leading to

$$
\tilde{i}_{p p}=\frac{V_{d c} T_{s}}{L}\left\{\left(m \cos \vartheta-\frac{1}{3}\right) \frac{\delta_{p}}{2}+\left(m \cos \vartheta-\frac{2}{3}\right) \delta_{1}\right\} .
$$

Introducing the expressions for $\delta_{p}$ and $\delta_{1}$ given in Table I and normalizing, the current ripple becomes

$$
r(m, \vartheta)=\left(u_{\alpha}-\frac{1}{3}\right)\left[1-\frac{3}{2}\left(u_{\alpha}-\frac{1}{\sqrt{3}} u_{\beta}\right)\right]+2\left(u_{\alpha}-\frac{2}{3}\right)\left(1-\sqrt{3} u_{\beta}\right),
$$

for triangle $I-2 b$

$$
r(m, \vartheta)=\left(u_{\alpha}-\frac{1}{3}\right)\left[2-\frac{3}{2}\left(u_{\alpha}+\frac{1}{\sqrt{3}} u_{\beta}\right)\right]+2\left(u_{\alpha}-\frac{2}{3}\right)\left(\sqrt{3} u_{\beta}-1\right),
$$

for triangle $\mathrm{I}-3 \mathrm{~b}$.

The obtained equation for the triangle 1-3b (yellow area) can be compared to the corresponding equation (4.22) obtained by the first method (A) of the current ripple evaluation, and also in this case it is matching.

3) Evaluation in the third sector

In the third sector, $\pi / 3 \leq \vartheta \leq \pi / 2$, two different sub-cases are identified, related to the two colored regions on Fig. 4.7.

The first sub-cases considering triangles II-1a and II-2a, corresponding to the yellow area in Fig. 4.7, are presented in diagram $\mathbf{0}$ of Fig. 4.8c, and in 
diagram $\mathbf{D}$ of Fig. 4.9c. In these sub-cases the switch configurations $\{++0\}$ for triangle II-1a, and $\left\{\begin{array}{ll}0 & 0\end{array}\right\}$ for triangle II-2a, with the corresponding dutycycle $\delta_{p} / 2$ for both, can be considered to evaluate $\tilde{i}_{p p}$, leading to

$$
\tilde{i}_{p p}=\frac{V_{d c} T_{s}}{L}\left\{\left(\frac{1}{3}-m \cos \vartheta\right) \frac{\delta_{p}}{2}\right\} .
$$

Introducing the expressions for $\delta_{p}$ given in Table I and normalizing by (4.8) and (4.16), the current ripple becomes

$$
\begin{gathered}
r(m, \vartheta)=\frac{3}{2}\left(\frac{1}{3}-u_{\alpha}\right)\left(u_{\alpha}+\frac{1}{\sqrt{3}} u_{\beta}\right), \text { for triangle II -1a }, \\
r(m, \vartheta)=\left(\frac{1}{3}-u_{\alpha}\right)\left[1-\frac{3}{2}\left(\frac{1}{\sqrt{3}} u_{\beta}-u_{\alpha}\right)\right], \text { for triangle II - 2a . }
\end{gathered}
$$

The second sub-cases are considering triangle II-3a, corresponding to the purple triangle in Fig. 4.7, are presented in diagrams $\mathbf{0}$ and 2 of Fig. 4.10c. In both sub-cases the switch configurations $\{++0\}$ and $\{0+-\}$, with the corresponding the duty-cycles $\delta_{p} / 2$ and $\delta_{1}$, can be considered to evaluate $\tilde{i}_{p p}$, leading to

$$
\tilde{i}_{p p}=\frac{V_{d c} T_{s}}{L}\left\{\left(m \cos \vartheta-\frac{1}{3}\right) \frac{\delta_{p}}{2}+m \cos \vartheta \delta_{1}\right\} .
$$

Introducing the expressions for $\delta_{p}$ and $\delta_{1}$ given in Table I and normalizing, the current ripple becomes

$$
r(m, \vartheta)=\left(u_{\alpha}-\frac{1}{3}\right)\left(2-\sqrt{3} u_{\beta}\right)+3 u_{\alpha}\left(\frac{1}{\sqrt{3}} u_{\beta}-u_{\alpha}\right) .
$$

The obtained equation for the triangle II-3a (purple area) is matching the corresponding equation (4.25) obtained by the first method (A) of the current ripple evaluation. 


\subsubsection{Peak-to-peak current ripple diagrams}

The behavior of the peak-to-peak current ripple amplitude in the fundamental period (first quadrant) is summarized in Figs. 4.11 and 4.12. In particular, the normalized current ripple $r(m, \vartheta)$ defined by $(4.18)$ is presented.

Fig. 4.11 shows $r(\vartheta)$ in the cases of $m=1 / 3,1 / 2,2 / 3,1$, and $2 / \sqrt{3}\left(m_{\max }\right)$, corresponding to dashed circles (blue) in Fig. 4.7. Crossings of the three main sectors are visible for modulation indexes $1 / 3,1 / 2$, and $2 / 3$. For $m=1$ six different regions are visible, whereas four different regions can be distinguished for the maximum modulation index $(2 / \sqrt{3})$. All these regions correspond to the different colored areas in Fig. 4.7.

In Fig. 4.12 the colored map of $r(m, \vartheta)$ in the first quadrant of the normalized output voltage vector $\left(u_{\alpha}-u_{\beta}\right.$ plane) is presented. The current ripple goes to zero in the surroundings of $m=0$, being mainly applied only the null vector. The same behavior can be noticed around all the output vectors, since at their tip the output voltage perfectly matches the reference voltage, and the current ripple becomes zero. The map shows a general continuous distribution of the current ripple amplitude. The only discontinuity is observed across the phase angle $\pi / 6$ (red line in Fig. 4.12), due to the change of the two pivot vectors $\left(\boldsymbol{v}_{p 1}\right.$ and $\boldsymbol{v}_{p 6}$, represented as two red dots).

By observing Fig. 4.11 it can be noticed that the maximum current ripple amplitude is almost constant for intermediate modulation indexes, i.e., $m \approx[0.1-$

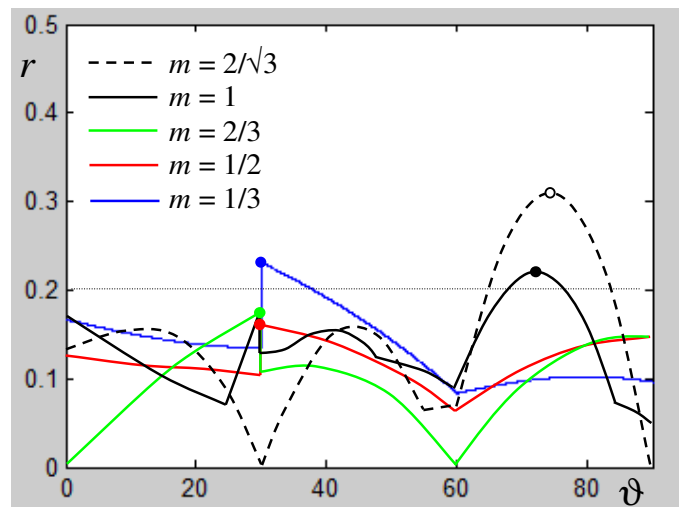

Fig. 4.11. Normalized peak-to-peak current ripple amplitude $r(m, \vartheta)$ in the first quadrant for different modulation indexes as a function of $\vartheta$. 


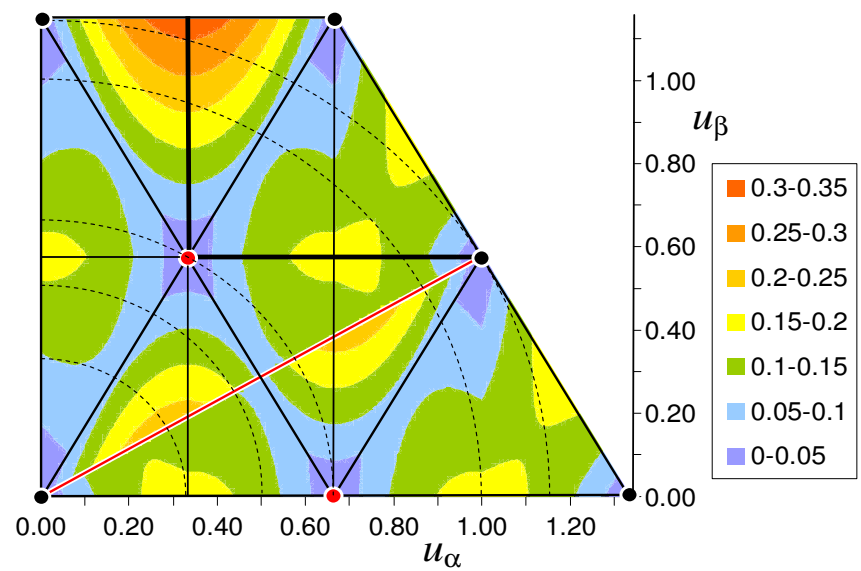

Fig. 4.12. Normalized peak-to-peak current ripple amplitude $r(m, \vartheta)$ in the first quadrant as a colored map in the normalized voltage plane $\alpha-\beta$.

$1]$, ranging around the value 0.2 , leading to the simplified expression $r^{\max } \approx 0.2$ or $\tilde{i}_{p p}^{\max } \approx \frac{V_{d c} T_{s}}{10 L}$ in the approximate range $0.1 \leq m \leq 1$.

The average of the normalized peak-to-peak current ripple in the fundamental period is presented in Fig. 4.13.

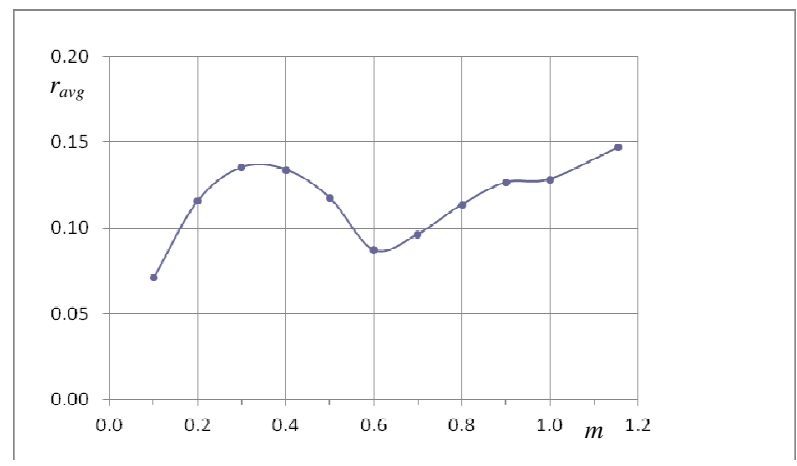

Fig. 4.13. The average of the normalized peak-to-peak current ripple amplitude as function of modulation index. 


\subsection{Numerical and experimental results}

In order to verify the theoretical developments shown in previous sections, circuit simulations and experimental tests are carried out. Circuit simulations are done by Sim-PowerSystems of Matlab considering three-phase inverter with ideal switched connected to sinusoidal voltage sources by RL impedance, having $R=4 \Omega$ and $L=24 \mathrm{mH}$. This topology could represent a general threephase inverter connected to either motor or grid. In all simulations the fundamental frequency is set to $50 \mathrm{~Hz}$, the switching frequency $1 / T_{s}$ is $2.1 \mathrm{kHz}$, and the dc voltage supply $V_{d c}$ is $600 \mathrm{~V}$. A centered symmetrical PD carrierbased PWM technique is implemented in both simulations and experiments, equivalent to centered NTV space vector modulation.

The experimental setup with custom-made three-level NPC inverter was used, as shown in Fig. 4.14. Power switches are Semikron SKM50GB12T4 IGBT modules, and clamping diodes are Semikron SKKD 46/12. DSpace ds1006 hardware has been employed for the real-time implementation of the algorithm. The total dc voltage $\left(2 V_{d c}\right)$, provided from the external dc source Sorensen SGI 600/25, was $600 \mathrm{~V}$. Switching frequency was set to $2.1 \mathrm{kHz}$ and the inverter's dead time of $6 \mu$ s (not compensated) is implemented in the

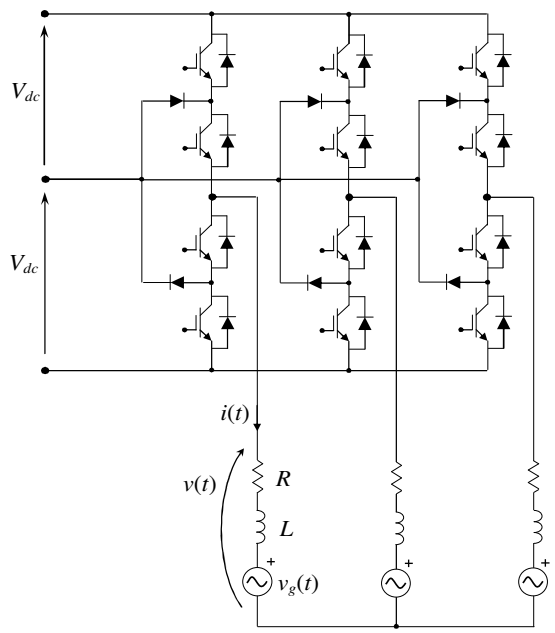

Fig. 4.14. Three-level NPC inverter supplying an RLE circuit, representing either a motor-load or a grid-connected applications. 
hardware. Fundamental frequency was kept at $50 \mathrm{~Hz}$ for easier comparison with analytical developments.

The experiments have been done by feeding a three-phase symmetrical induction motor (mechanically unloaded). A picture of the experimental setup is given in Fig. 4.15. Main motor parameters (all referred to stator) are: stator resistance $R_{s}=2.4 \Omega$, rotor resistance $R_{r}{ }^{\prime}=1.6 \Omega$, stator leakage inductance $L_{l s}$ $=12 \mathrm{mH}$, rotor leakage inductance $L_{l r}{ }^{\prime}=12 \mathrm{mH}$, magnetizing inductance $L_{m}=$ $300 \mathrm{mH}$, pole pairs $p=2$. According to the model of induction motor for higher order harmonics, which are determining the current ripple, the equivalent inductance $L=L_{l s}+L_{l r}{ }^{\prime}=24 \mathrm{mH}$ is considered for the ripple evaluation.

Tektronix oscilloscope MSO2014 with current probe TCP0030 was used for the measurements, and built-in noise filter (cut-off frequency $f_{c}=600 \mathrm{kHz}$ ) was applied. In Fig. 4.16 is given a screenshot showing an example of voltage and current waveforms. A further low-pass filter $\left(f_{c}=25 \mathrm{kHz}\right)$ was applied in post-processing of the experimental data to better clean the waveforms. The instantaneous current ripple $\tilde{i}$ in both simulations and experiments is calculated as the difference between the instantaneous current and its fundamental component, i.e.

$$
\tilde{i}(t)=i(t)-I_{\text {fund }}(t) \text {. }
$$
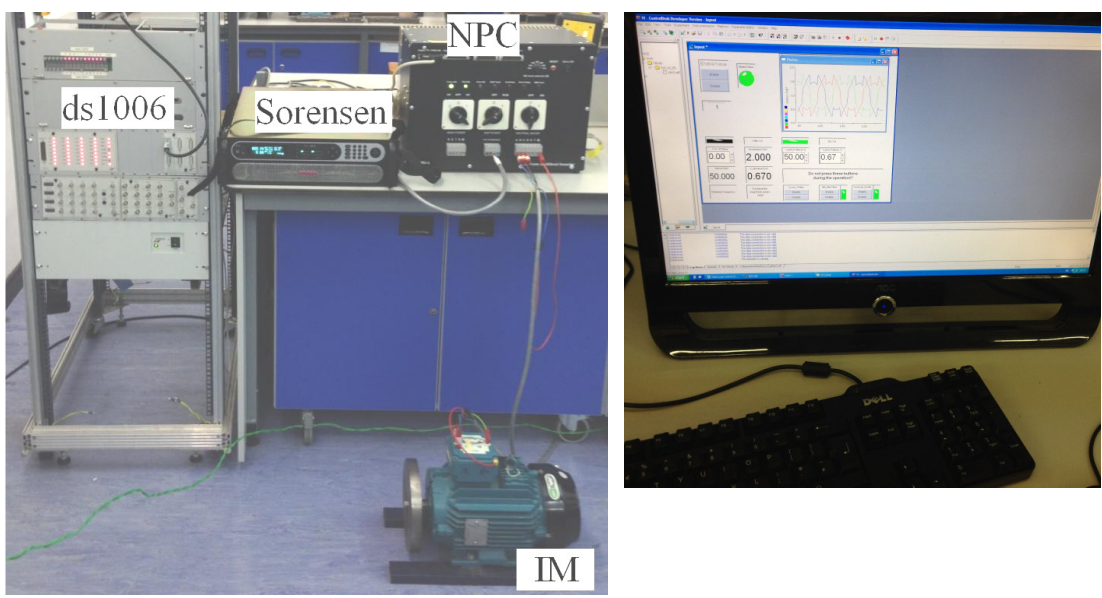

Fig. 4.15. Experimental setup 


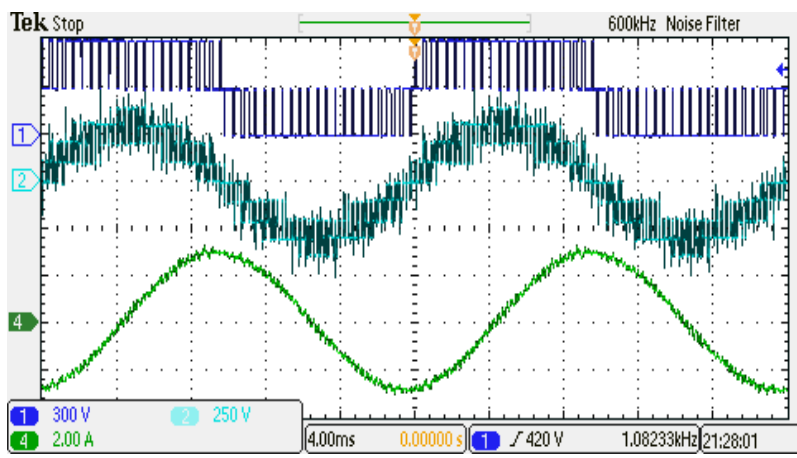

Fig. 4.16. Example of output voltage and current waveforms from the scope for $m=1$ (Ch1: leg voltage, $\mathrm{Ch} 2$ : phase voltage, $\mathrm{Ch} 4$ : phase current).

The three-phase system is well balanced and the first phase is selected for further analysis, as in analytical developments. Different values of $m$ have been investigated $\left(1 / 3,1 / 2,2 / 3,1\right.$, and $\left.2 / \sqrt{ } 3=m_{\max }\right)$.

In Figs. 4.17, 4.19, 4.21, 4.23, and 4.25 is shown the current ripple obtained with simulations (a) and experiments (b), for different modulation indexes, respectively. The ripple in experiments is processed as explained above. Comparison is made with the half of the peak-to-peak current ripple, $\tilde{i}_{p p} / 2$. Positive and negative envelope (blue traces) are determined by the equations presented in Section 4.2. The corresponding experimental results are shown as gray traces.

In the corresponding Figs. 4.18, 4.20, 4.22, 4.24, and 4.26 is depicted the instantaneous output current obtained in simulations (a) and experiments (b) with different modulation indexes, respectively. Current is shown with the calculated upper/lower ripple envelope, depicted in blue colors. The corresponding experimental results are shown as gray traces.

The values of the modulation index have been chosen to cover all possible sub-cases (different colored regions in Fig. 4.5). The agreement is almost good in the whole fundamental period, proving the effectiveness of the analytical developments. The small mismatches, which can be observed in the experimental results, could be the consequence of the inverters dead-time that has not been compensated, but this aspect can be further examined. 
(a)
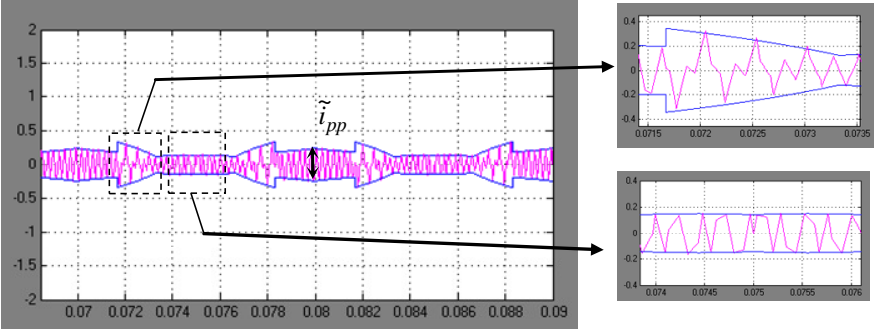

(b)

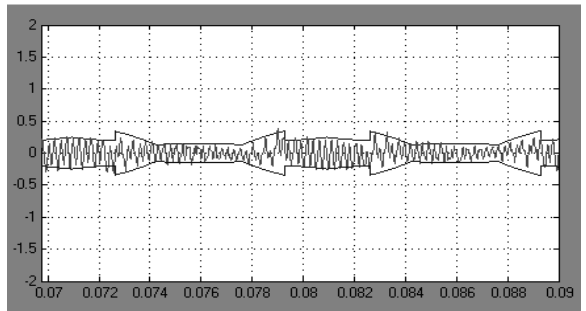

Fig. 4.17. Current ripple (pink), $m=1 / 3$, and evaluated peak-to-peak envelope (blue) for one fundamental period: (a) simulation, with details, and (b) experimental results.

(a)

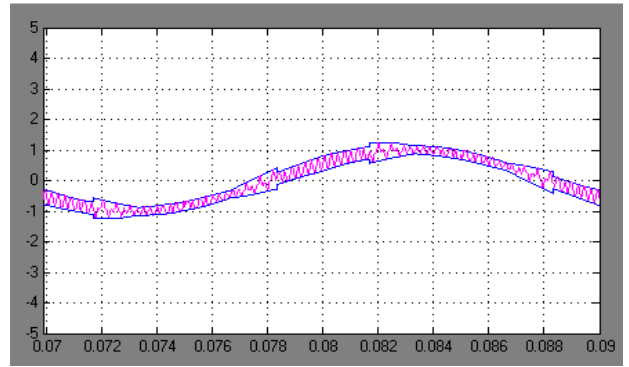

(b)

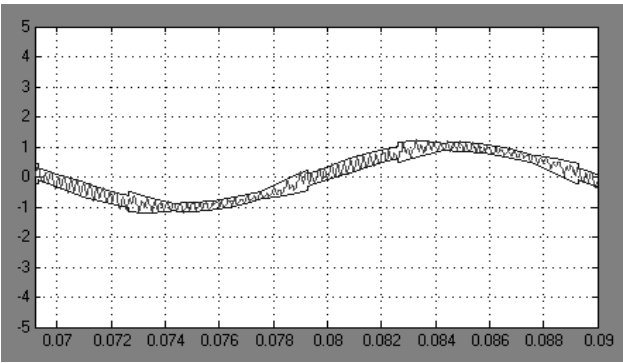

Fig. 4.18. Instantaneous output current (pink) with calculated current envelopes (blue traces) for $m=1 / 3$ : (a) simulated, (b) experimental results. 
(a)
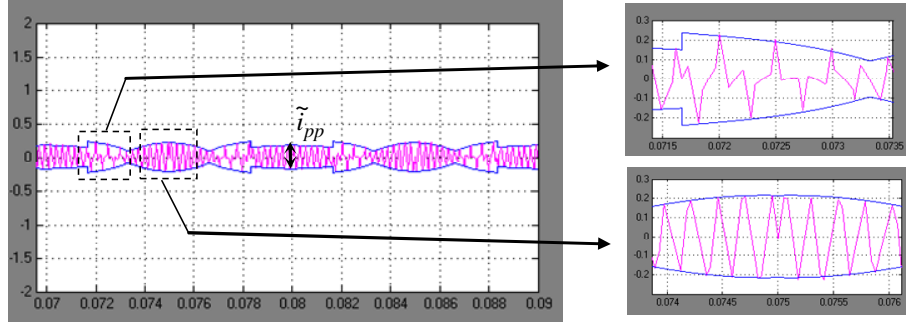

(b)

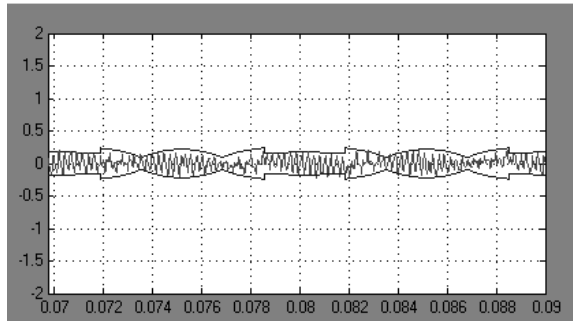

Fig. 4.19. Current ripple (pink), $m=1 / 2$, and evaluated peak-to-peak envelope (blue) for one fundamental period: (a) simulation, with details, and (b) experimental results.

(a)

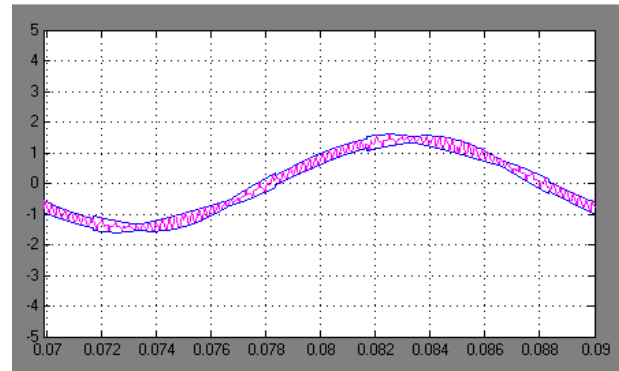

(b)

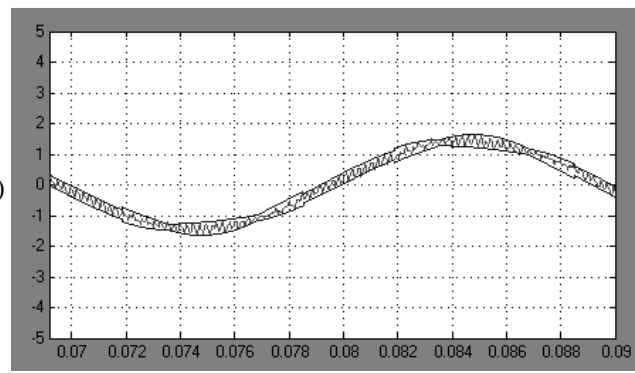

Fig. 4.20. Instantaneous output current (pink) with calculated current envelopes (blue traces) for $m=1 / 2$ : (a) simulated, (b) experimental results. 
(a)
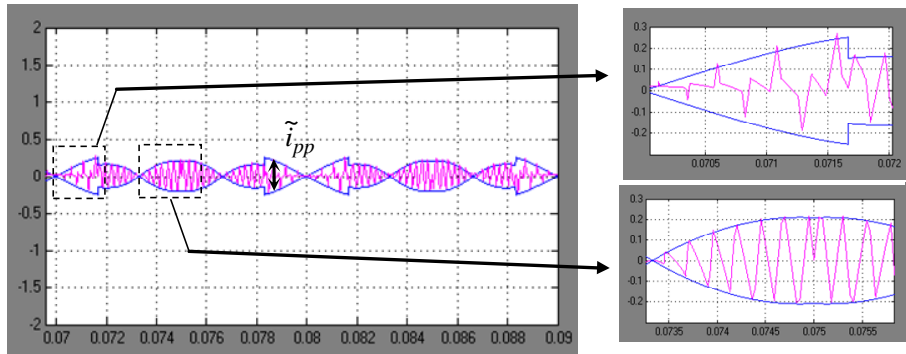

(b)
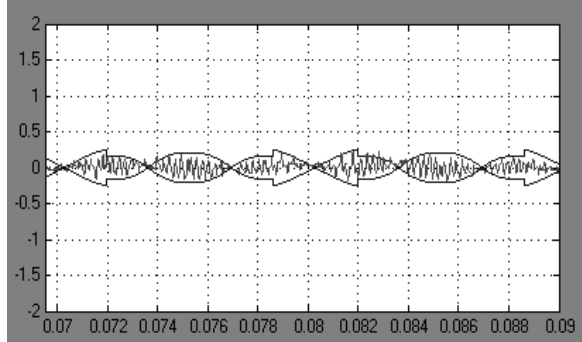

Fig. 4.21. Current ripple (pink), $m=2 / 3$, and evaluated peak-to-peak envelope (blue) for one fundamental period: (a) simulation, with details, and (b) experimental results.

(a)

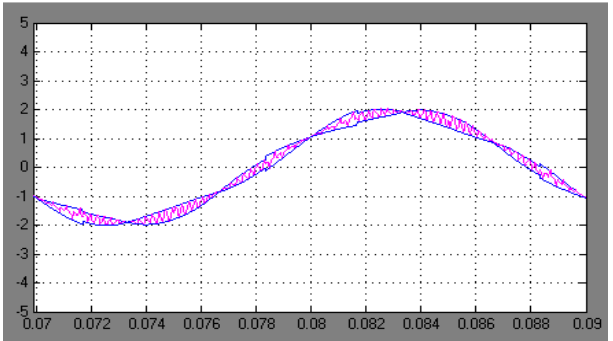

(b)

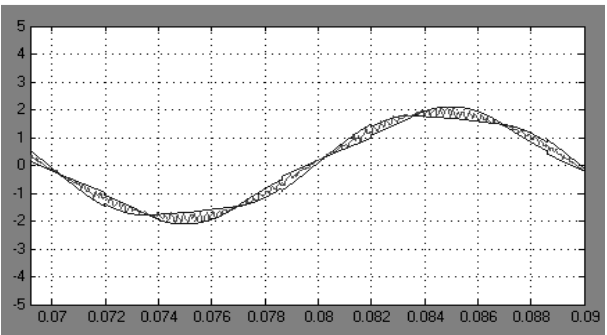

Fig. 4.22. Instantaneous output current (pink) with calculated current envelopes (blue traces) for $m=2 / 3$ : (a) simulated, (b) experimental results. 
(a)

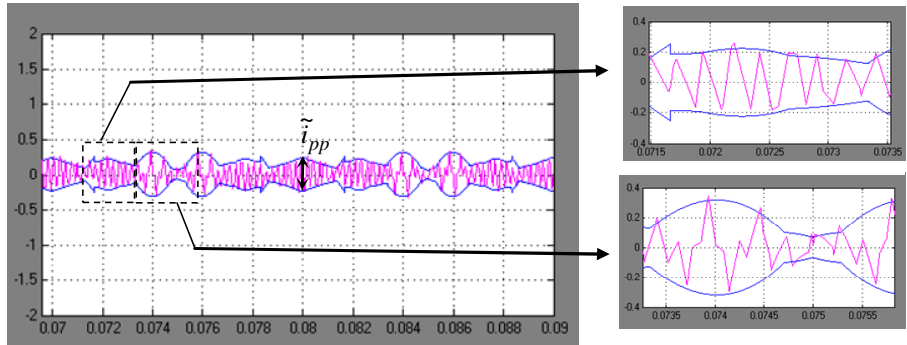

(b)

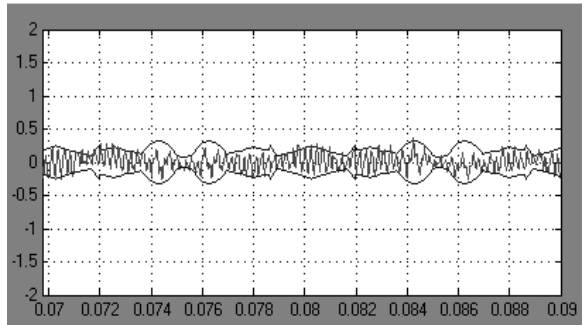

Fig. 4.23. Current ripple (pink), $m=1$, and evaluated peak-to-peak envelope (blue) for one fundamental period: (a) simulation, with details, and (b) experimental results.

(a)

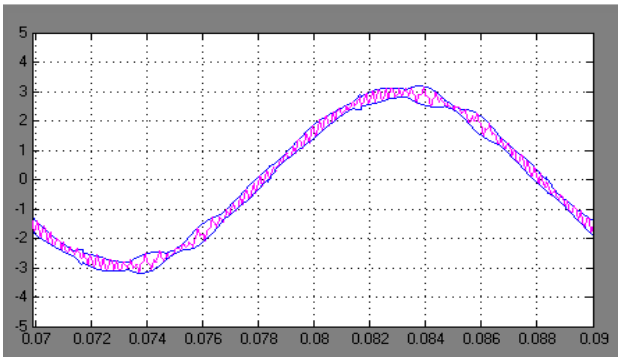

(b)

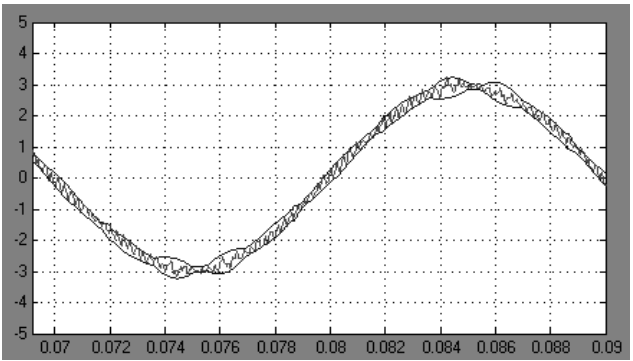

Fig. 4.24. Instantaneous output current (pink) with calculated current envelopes (blue traces) for $m=1$ : (a) simulated, (b) experimental results. 
(a)
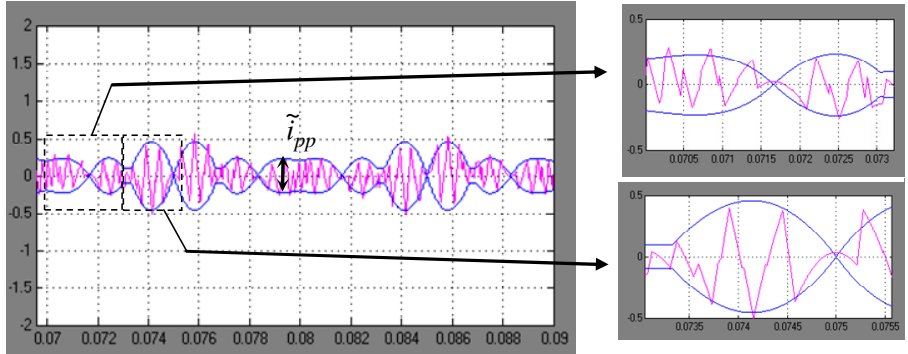

(b)

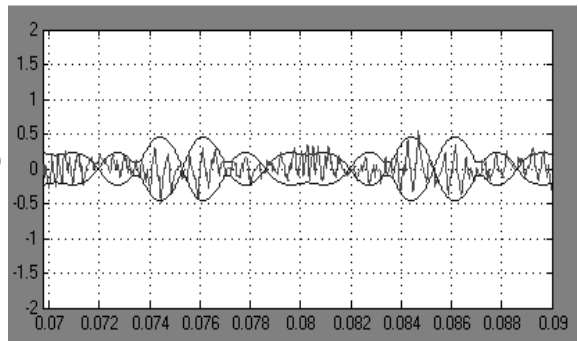

Fig. 4.25. Current ripple (pink), $m=2 / \sqrt{3}$, and evaluated peak-to-peak envelope (blue) for one fundamental period: (a) simulation, with details, and (b) experimental results.

(a)

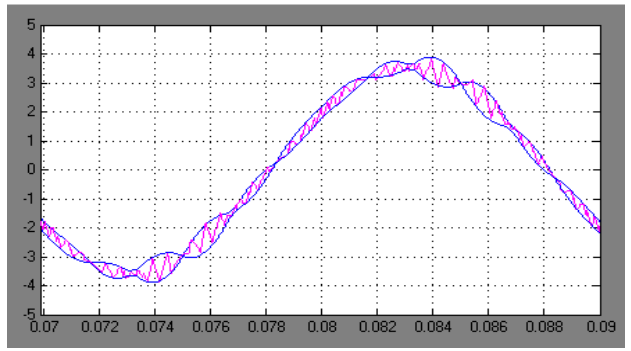

(b)

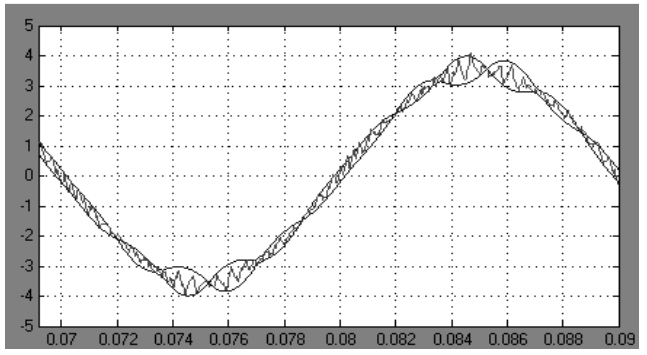

Fig. 4.26. Instantaneous output current (pink) with calculated current envelopes (blue traces) for $m=2 / \sqrt{3}$ : (a) simulated, (b) experimental results. 


\subsection{References}

[1] X. Yuan, I. Barbi, "Fundamentals of a new diode clamping multilevel inverter," IEEE Trans. Power Electron., vol. 15, no. 4, pp. 711-718, July 2000 .

[2] D. Ostojic , "A multilevel converter structure for grid-connected PV plants," PhD thesis, University of Bologna, Italy, March 2010.

[3] M. Malinowski, K. Gopakumar, J. Rodriguez, M. Perez, "A survey on cascaded multilevel inverters," IEEE Trans. Ind. Electron., vol. 57, no. 7, pp. 2197-2206, July 2010.

[4] J. Rodriguez, J.S. Lai, F.Z. Peng, "Multilevel inverters: a survey of topologies, controls and applications," IEEE Trans. on Industrial Electronics, vol. 49, no. 4, pp. 724-738, August 2002.

[5] Y.H. Lee, D.H. Kim, D.S. Hyun, "Carrier based SVPWM method for multi-level system with reduced HDF," In Proc. of Industry Applications Conference, Rome IT, 8-12 Oct. 2000, vol. 3, pp. 1996-2003.

[6] D. Casadei, G. Serra, A. Tani, L. Zarri, "Cascaded three-level inverter modulation strategies: a unifying point of view based on duty-cycle space vector approach," In Proc. of International Symposium on Power Electronics, Electrical Drives, Automation and Motion (SPEEDAM), Taormina IT, 23-26 May 2006, pp. 583-590.

[7] X. Zhang, J. Spencer, "Study of multisamled multilevel inverters to improve control performance," IEEE Trans. on Power Electronics, vol. 27, no. 11, pp. 4409-4416, November 2012.

[8] B. Vafakhah, J. Salmon, A.M. Knight, "A new space-vector PWM with optimal switching selection for multilevel coupled inductor inverters," IEEE Trans. on Ind. Electronics, vol. 57, no. 7, pp. 2354-2364, July 2010.

[9] S. Wei, B. Wu, F. Li, C. Liu, "A General Space Vector PWM Control Algorithm for Multilevel Inverters," In Proc. of $18^{\text {th }}$ IEEE Annual IEEE Applied Power Electronics Conference and Exposition (APEC), Miami Beach FL, 9-13 Feb. 2003, vol. 1, pp. 562-568. 
[10] P.C. Loh, D.G. Holmes, Y. Fukuta, T.A Lipo, "Reduced common-mode modulation strategies for cascaded multilevel inverters," IEEE Trans. on Ind. Applications, vol. 39, no. 5, pp. 1386-1395, Sept/Oct. 2003.

[11] B.P. McGrath, D.G. Holmes, T.A. Lipo, "Optimized space vector switching sequences for multilevel inverters," IEEE Trans. on Power Electronics, vol. 18, no. 6, pp. 1293-1301, Nov. 2003.

[12] T. Bruckner, D.G. Holmes, "Optimal pulse-width modulation for threelevel inverters," IEEE Trans. on Power Electronics, January 2005, vol. 20, no. 1, pp. 82-89.

[13] B.P. McGrath, D.G. Holmes, T. Meynard, "Reduced PWM harmonic distortion for multilevel inverters operating over a wide modulation range," IEEE Trans. on Power Electronics, vol. 21, no. 4, pp. 941-949, July 2006.

[14] F. Wang, "Sine-triangle versus space-vector modulation for three-level PWM voltage-source inverters," IEEE Trans. on Industry Applications, vol. 38, no. 2, pp. 500-506, March/April 2002.

[15] W.Yao, H. Hu, Z. Lu, "Comparison of space-vector modulation and carrier-based modulation of multilevel inverter," IEEE Trans. on Power Electronics, vol. 23, no. 1, pp. 45-51, January 2008.

[16] S. Das, G. Narayanan, "Novel switching sequences for a space-vectormodulated three-level inverter," IEEE Trans. on Ind. Electronics, vol. 59, no. 3, pp. 1477-1487., March 2012.

[17] S. Srinivas, K. R. Sekhar, "Theoretical and experimental analysis for current in a dual-inverter-fed open-end winding induction motor drive with reduced switching PWM," IEEE Trans. on Ind. Electronics, vol. 60, no. 10, pp. 4318-4328, October 2013.

[18] D. Jiang, , F. Wang, "Current-ripple prediction for three-phase PWM converters," IEEE Trans. on Ind. Applications, vol. 50, no. 1, pp. 531538, Jan/Feb 2014.

[19] D. Jiang, F. Wang, "A general current ripple prediction method for the multiphase voltage source converter," IEEE Trans. on Power Electronics, vol. 29, no. 6, pp. 2643-2648, June 2014. 


\subsection{Authored papers}

- G. Grandi, J. Loncarski, O. Dordevic, "Analytical evaluation of output current ripple amplitude in three-phase three-level inverters," submitted to IET Power Electronics Journal, 2014.

- G. Grandi, J. Loncarski, "Implementation of carrier-based centered PWM in three-phase three-level inverter," submitted to Speedam Conference, Italy, 2014. 


\section{Comparison of the peak-to-peak current ripple}

\subsection{Comparison between different phase number}

The analysis of peak-to-peak output current ripple developed in the previous chapters for inverters with different phase number is compared. Reference is made to centered and symmetrical switching patterns, generated either by CBor SV-PWM, being the optimal modulation in terms of the output current RMS minimization. The peak-to-peak ripple is evaluated by the expressions presented for specific case in the previous chapters and is given as the function of the modulation index. Several peak-to-peak ripple diagrams are presented for each case and compared. Maximum of the peak-to-peak current ripple and the simplified expressions for its evaluation are compared among cases with different number of phases. The instantaneous current ripple is introduced for a generic balanced multiphase loads consisting of series RL impedance and ac back emf (RLE circuit). In addition, the derived conclusions from the comparison are verified on realistic inverter-RL load circuit model, as represented in Fig. 5.1, in numerical simulations, implemented by the Simulink tool of Matlab.

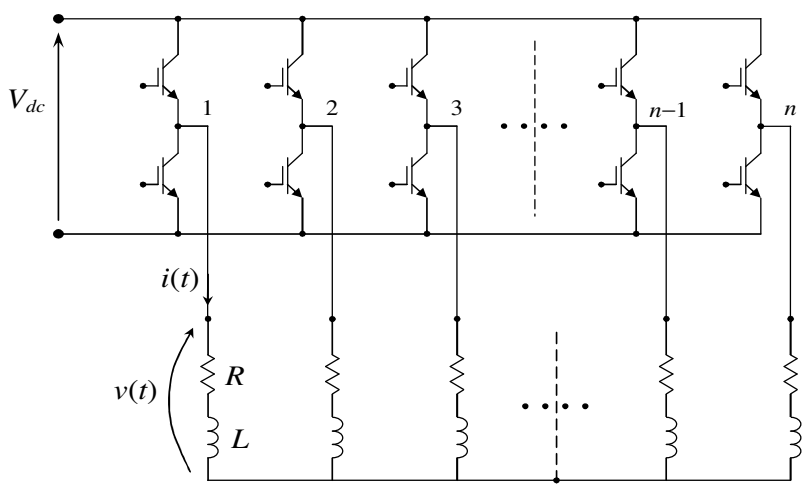

Fig. 5.1. Multiphase VSI supplying a RL load. 
5.1.1. Review of peak-to-peak current ripple evaluation in multiphase VSIs

For the $n$-phase system $\left\{x_{1}, x_{2}, x_{3}, \ldots x_{n}\right\}$, the $(n-1) / 2$ space vectors, used to represent voltage and current phase quantities, lie in the planes $\alpha_{1}-\beta_{1}$, $\alpha_{3}-\beta_{3}, \ldots, \alpha_{n-2}-\beta_{n-2}$, respectively, and are expressed as

$$
\begin{gathered}
x_{0}=\frac{1}{n} \sum_{k=1}^{n} x_{k}, \\
x_{h}=\frac{2}{n} \sum_{k=1}^{n} x_{k} \alpha^{h(k-1)}, h=1,3, \ldots, n-2
\end{gathered}
$$

being $\alpha=\exp (j 2 \pi / n)$. In (5.1) $x_{0}$ is the zero-sequence (homopolar) component, equal to zero in balanced systems, and in (5.2) $\boldsymbol{x}_{h}$ is the $h$-th component of multiple space vectors.

The inverse multiple space vector transformations are

$$
x_{k}=x_{0}+\sum_{h=1}^{n} x_{h} \cdot \alpha^{h(k-1)}, \quad k=1,2, \ldots, n, \quad h=1,3, \ldots, n-2 .
$$

The SV-PWM of multiphase inverters is based on the determination of application times of active and null inverter voltage vectors $\boldsymbol{v}_{1}, \boldsymbol{v}_{3}, \ldots, \boldsymbol{v}_{n-2}$ in every switching period $T_{s}$. In the case of symmetrical SV-PWM, the sequence is determined in $T_{s} / 2$ and it is repeated symmetrically in the next half of the switching period. As result of the SV-PWM, for each phase, the average of the inverter output voltage $\bar{v}\left(T_{s}\right)$ corresponds to the reference voltage $v^{*}$.

In the case of sinusoidal balanced output voltages supplying a balanced load, the zero-sequence component is null. In this case, introducing the modulation index $m=V^{*} / V_{d c}$, the reference space voltage vectors become

$$
\left\{\begin{array}{l}
v_{1}^{*}=v^{*}=m V_{d c} e^{j \vartheta} \\
v_{3}^{*}=0 \\
\cdots \\
v_{n-2}^{*}=0 .
\end{array}\right.
$$


The considered SV-PWM is quarter-wave symmetric, and it can be analyzed in the range $[0, \pi / 2]$ of the phase angle $\vartheta=\omega t$. The calculation of application times for the first sector (1) $k=1$ ) defined in Chapter 2 for three-phase, and in Chapter 3 for five- and seven-phase VSIs, can be extended to any sector $k$ by replacing the phase angle $\vartheta$ with $\vartheta-(k-1) \pi / n, k=1,2, \ldots, 2 n$. Note that the modulation limit is defined according to the generalized expression for $n$ phases, $m_{\max }=[2 \cos (\pi / 2 n)]^{-1}$.

In Fig. 5.2 are presented the space vector diagrams of the output voltage in the first $\alpha-\beta$ plane for three-, five-, and seven-phase inverters with emphasized the switch configurations and the corresponding voltage vectors involved in the commutation process in the first quadrant. The different colors represent the different sub-cases identified for the current ripple evaluation, as explained in details in Chapters 2 and 3.

Due to the symmetry among all phases in the considered case of sinusoidal balanced currents, only the $1^{\text {st }}$ phase is examined in the analysis. In terms of multiple space vectors, the phase variables are given by (5.3). For the $1^{\text {st }}$ phase, it results in the sum of the projections of the $(n-1) / 2$ space vectors on the real axes. In particular, introducing (5.4) in (5.3), the average output voltage of the $1^{\text {st }}$ phase is given by

$$
\bar{v}\left(T_{s}\right)=v^{*}=\sum_{h=1}^{n-2} \Re e\left\{v_{h}^{*}\right\}=m V_{d c} \cos \vartheta, h \text { odd } .
$$

By introducing (5.5) in the expression for alternating component of inverter

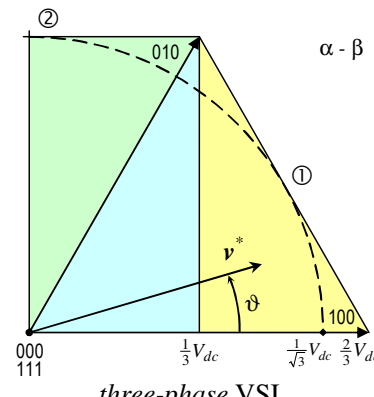

three-phase VSI

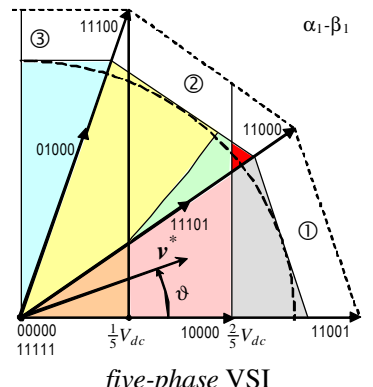

five-phase VSI

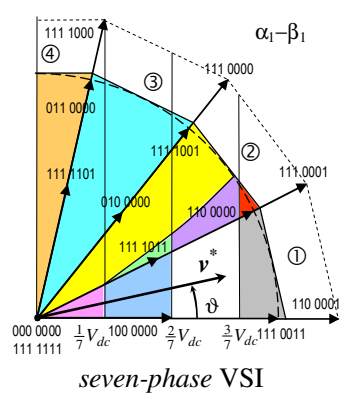

seven-phase VSI

Fig. 5.2. Space vector diagrams of inverter output voltage in the range $\vartheta=\left[0,90^{\circ}\right]$. Different colored areas correspond to different equations for determining the current ripple. 
voltage (2.6) and calculating $v(t)$ by (5.3), the alternating component of inverter output voltage (first phase) for multiphase inverters can be written as

$$
\tilde{v}(t)=\left[S_{1}-\frac{1}{n}\left(S_{1}+S_{2}+\ldots+S_{n}\right)\right] V_{d c}-m V_{d c} \cos \vartheta,
$$

where $S_{k}$ represents the switch state of the $k$-th inverter leg, $S_{k}=[0,1]$.

For the current ripple evaluation in the whole phase angle range $0<\vartheta<\pi / 2$, the different cases corresponding to the different sectors (1), (2), ..., depicted in Fig. 5.2 were considered, for three-, five- and seven-phase inverters, respectively. Additional sub-cases, also determined by the value of modulation index, are identified in Fig. 5.2 with different colored areas. Peak-to-peak current ripple has been determined on the basis of the instantaneous current ripple and its peak-to-peak value, (2.10) and (2.11), and considering (5.6) in a specific sub-case, following the procedure for three-phase and multiphase inverters in previous chapters.

\subsubsection{Comparison of the peak-to-peak current ripple diagrams}

In order to show the behavior of the peak-to-peak current ripple amplitude in the fundamental period for the considered cases, in Figs. 5.3 and 5.4 is represented the normalized function of the current peak-to-peak ripple amplitude, $r(m, \vartheta)$, defined as

$$
\tilde{i}_{p p}=\frac{V_{d c} T_{s}}{2 L} r(m, \vartheta) .
$$

Fig. 5.3 shows $r(\vartheta)$ for $m=1 / 6,1 / 3$, and 1/2 (from left to right), for three-, five-, and seven-phase inverters (blue, red, and green lines, respectively). For low modulation indexes can be noted that inverters have similar ripple amplitude, while more difference rises with higher modulation indexes. In general, the ripple for the three-phase inverter is lower, whereas five- and seven-phase inverters have more similar ripple behavior, even for the higher modulation indexes.

Fig. 5.4 shows the colored map of $r(m, \vartheta)$ for the first quadrant of the $\alpha-\beta$ plane within the modulation limits for three-, five-, and seven-phase inverters, respectively. It can be noted that ripple amplitude is obviously zero for $m=0$ 

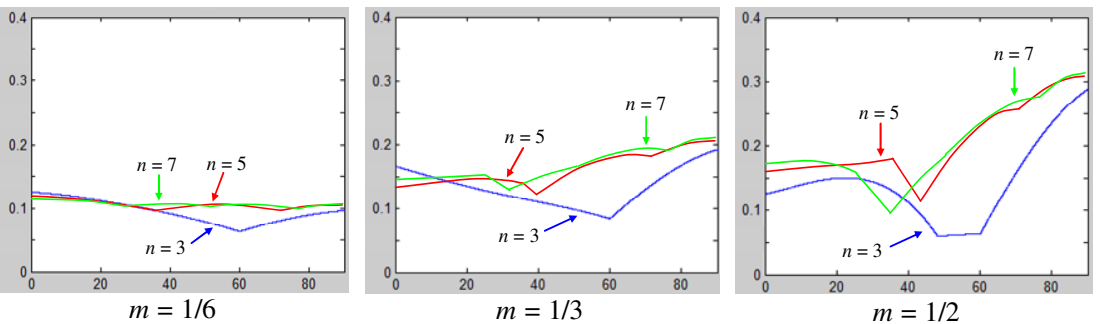

Fig. 5.3. Normalized peak-to-peak current ripple amplitude $r(m, \vartheta)$ for three-, five-, and sevenphase VSIs in the range $\vartheta=\left[0,90^{\circ}\right]$ for different modulation indexes.

in all cases, since the null configurations are the only applied, increasing almost proportionally with $m$ in the neighborhoods of $m=0$. A phase angle with minimum ripple can be indentified in the range $\vartheta \approx 50^{\circ} \div 60^{\circ}$ for threephase inverter, $\vartheta \approx 40^{\circ} \div 45^{\circ}$ for five-phase inverter, and $\vartheta \approx 30^{\circ} \div 35^{\circ}$ for sevenphase inverter. The phase angle with maximum ripple is $\vartheta=90^{\circ}$ for all cases, with ripple amplitude almost proportional to the modulation index. This aspect is developed in more details in the following.

On the basis of previous Figs. 5.3 and 5.4, the maximum of the normalized current ripple can be determined. The maximum of the current ripple can be evaluated in the phase angle range $\left[0,90^{\circ}\right]$ as described in Section 2.3.4 for three-phase, in Section 3.2.4 for five-phase, and in Section 3.4.4 for sevenphase inverters. The composition of the two local maxima of the normalized current ripple is given in Fig. 5.5, leading to the global maximum $r^{\max }$, for

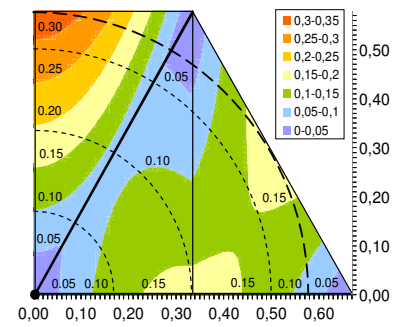

three-phase VSI

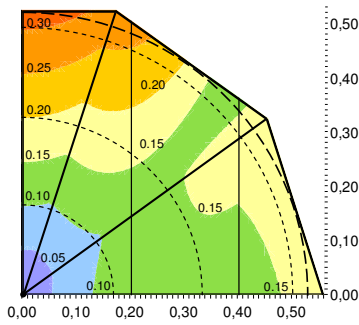

five-phase VSI

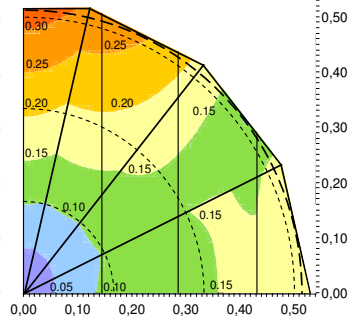

seven-phase VSI

Fig. 5.4. Map of the normalized peak-to-peak current ripple amplitude $r(m, \vartheta)$ for multiphase VSIs. 
three-, five-, and seven-phase inverters. The three vertical dashed gray lines represent the specific modulation indexes, $m=1 / 6,1 / 3$, and $1 / 2$. Similar behavior of the five- and seven-phase inverters can be noted in the maximum ripple as well. Maximum ripple is almost proportional to the modulation index, just the numeric coefficient is slightly different for different phase number. Then, a simplified expression for maximum of peak-to-peak current ripple amplitude can be obtained for the three-, five-, and seven-phase inverters as

3-phase VSI: $\tilde{i}_{p p}{ }^{\max } \cong \frac{V_{d c} T_{s}}{3.46 L} m, r^{\max } \cong 0.578 \mathrm{~m}$,

5-phase VSI: $\quad \tilde{i}_{p p}^{\max } \cong \frac{V_{d c} T_{s}}{3.25 L} m, r^{\max } \cong 0.615 \mathrm{~m}$,

7-phase VSI: $\quad \tilde{i}_{p p}^{\max } \cong \frac{V_{d c} T_{s}}{3.2 L} m, r^{\max } \cong 0.625 \mathrm{~m}$.

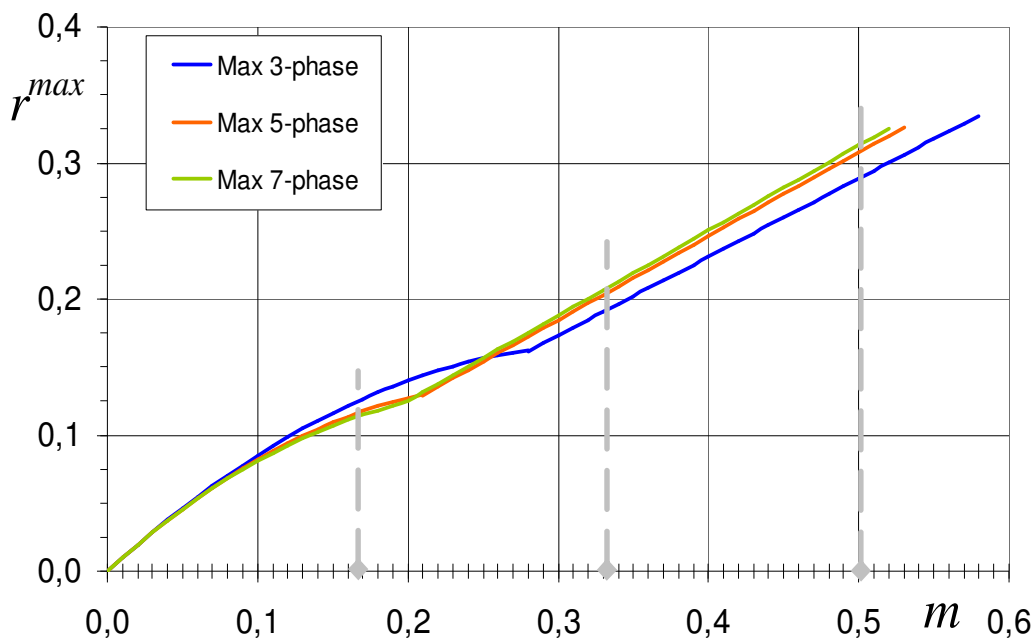

Fig. 5.5. Maximum of the normalized peak-to-peak current ripple amplitude as function of modulation index. 


\subsubsection{Numerical results}

In order to verify the comparison in the cases of inverters with different phase number, circuit simulations are carried out by Sim-PowerSystems of Matlab considering a balanced RL load, having $R=20 \Omega$ and $L=3 \mathrm{mH}$. In all simulations the fundamental frequency $f$ is set to $50 \mathrm{~Hz}$, the switching frequency $1 / T_{s}$ is $5.25 \mathrm{kHz}$, and the dc voltage supply $V_{d c}$ is $600 \mathrm{~V}$. A centered symmetrical carrier-based PWM technique is considered, equivalent to the multiple centered space vector modulation.

The instantaneous current ripple $\tilde{i}$ is calculated in simulations as the difference between the instantaneous current $i(t)$ and its fundamental component $I_{\text {fund }}(t)$, for the first phase, i.e.

$$
\tilde{i}(t)=i(t)-I_{\text {fund }}(t) .
$$

In all simulations the systems are well balanced and the first phase is selected for the analysis, as in analytical considerations. Different values of $m$ are investigated $(1 / 6,1 / 3$, and $1 / 2)$.

In Figs. 5.6a, 5.7a, and 5.8a (left columns) the current ripple $\tilde{i}$ obtained in simulations by (5.11) (pink trace), is compared with the half of peak-to-peak current ripple obtained by the analytical developments, $\tilde{i}_{p p} / 2$ (blue traces), for three-, five-, and seven-phase inverters in one fundamental period, for different modulation indexes.

In the corresponding Figs. 5.6b, 5.7b, and 5.8b (right columns) are depicted the instantaneous output currents (pink trace) with the calculated upper/lower ripple envelope, depicted in blue colors, for different modulation indexes.

The considered modulation indexes cover some of the possible sub-cases, i.e., colored regions in Fig. 5.2. Also in the simulation results the conclusions made in previous section can be observed. The agreement is good in the whole fundamental period, for all cases. 

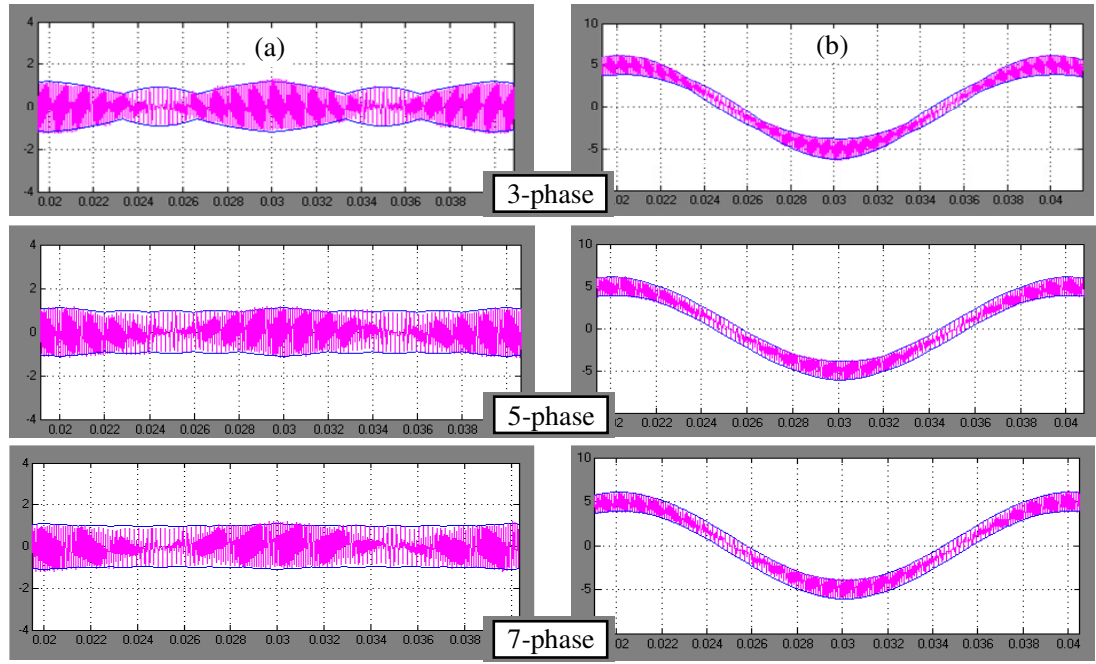

Fig. 5.6. Simulation results and calculated current ripple envelopes, $m=1 / 6$ : (a) simulated current ripple (pink) with evaluated peak-to-peak amplitude (blue); (b) simulated instantaneous current (pink) with calculated ripple envelopes (blue).
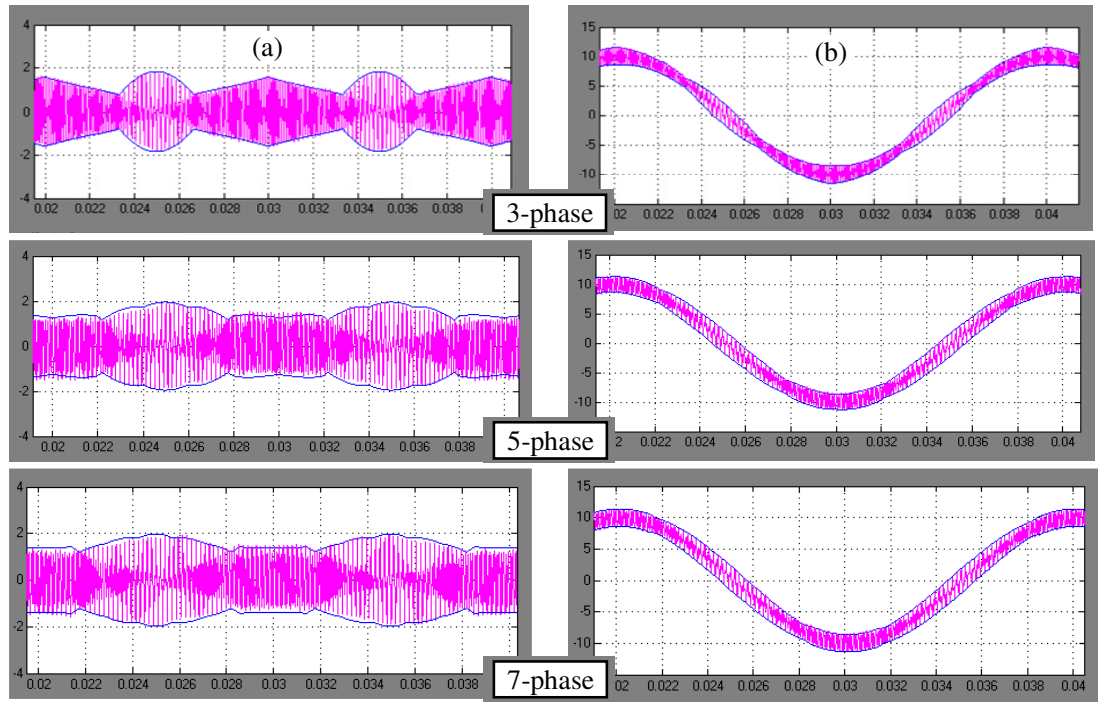

Fig. 5.7. Simulation results and calculated current ripple envelopes, $m=1 / 3$ : (a) simulated current ripple (pink) with evaluated peak-to-peak amplitude (blue); (b) simulated instantaneous current (pink) with calculated ripple envelopes (blue). 

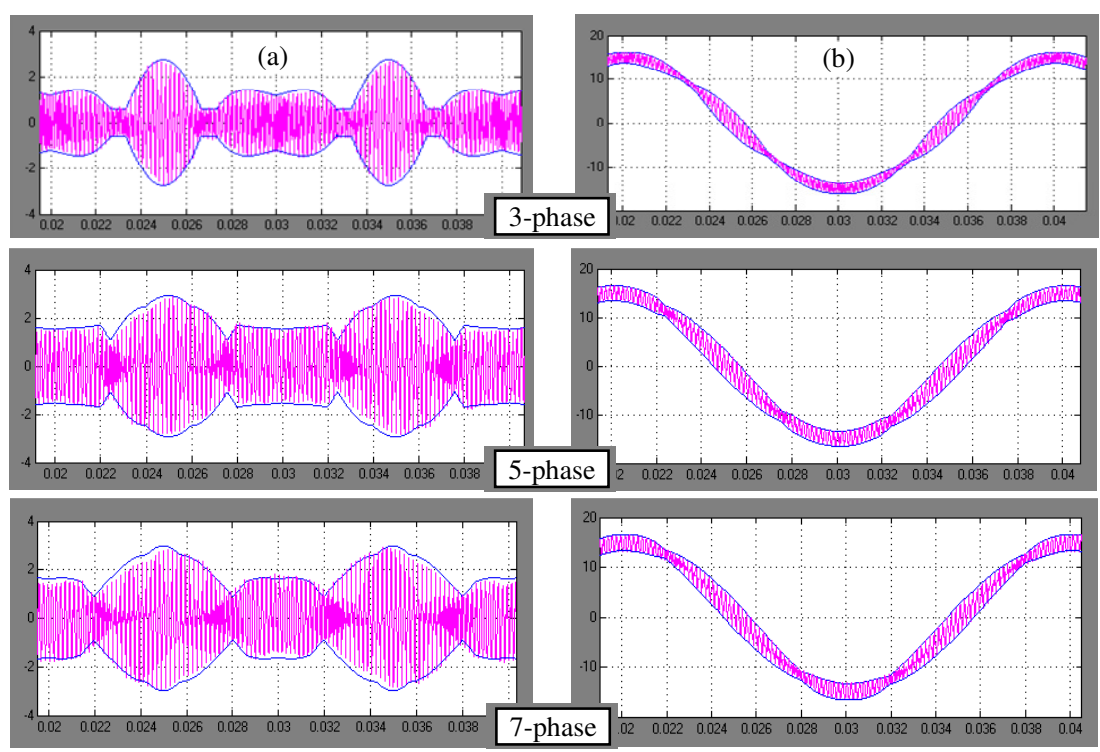

Fig. 5.8. Simulation results and calculated current ripple envelopes, $m=1 / 2$ : (a) simulated current ripple (pink) with evaluated peak-to-peak amplitude (blue); (b) simulated instantaneous current (pink) with calculated ripple envelopes (blue). 


\subsection{Comparison between different number of levels}

The comparison of the output current ripple amplitude in two- and threelevel inverters is presented in this section, by considering the same range of the output voltage for the two inverters. This means that for the two-level inverter the double of dc voltage is considered, as shown in Fig. 5.9. For the three-level inverter comparison can be made considering either the H-bridge or NPC configuration, as presented in Fig. 5.10. To have the same range of the output voltage as in two-level inverter, for the total supply of the NPC inverter is considered the double of dc voltage, $2 V_{d c}$. The peak-to-peak ripple amplitude is evaluated as described in the previous chapters for specific case and is given as the function of the modulation index over a fundamental period. Reference is made to optimal modulation, so-called centered PWM, obtained by both carried-based phase disposition modulation or nearest three vector space vector modulation. The instantaneous current ripple is determined for a generic balanced three-phase load consisting of series RL impedance and ac back emf (RLE), representing both motor-load and grid-connected applications. In addition, the conclusions made by the comparison are verified by numerical simulations on a realistic inverter-RL load circuit model implemented by the Simulink tool of Matlab.

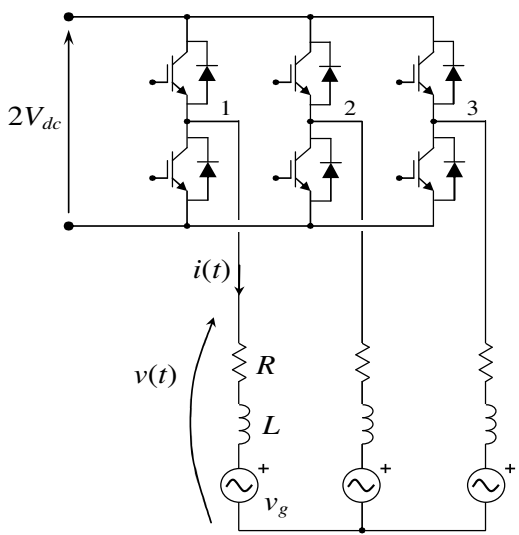

Fig. 5.9. Two-level inverter supplying an RLE circuit, representing either a motor-load or a grid-connected application 


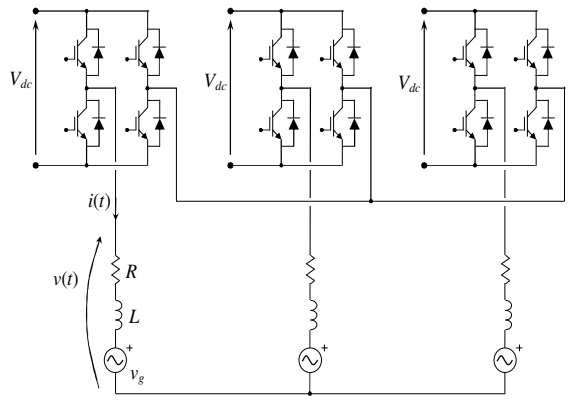

H-bridge configuration

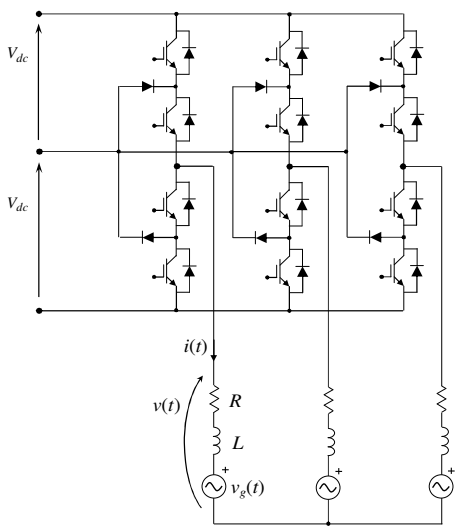

NPC configuration

Fig. 5.10. Three-level inverter supplying an RLE circuit, representing either a motor-load or a grid-connected application

5.2.1. Review of peak-to-peak current ripple evaluation in multilevel inverters

The output voltage vector $\boldsymbol{v}$ of a two-level inverter can be written considering the switching states of the $k$-th inverter phase, $S_{k}=[0,1]$, as

$$
\boldsymbol{v}=\frac{4}{3} V_{d c}\left[S_{1}+S_{2} \boldsymbol{\alpha}+S_{3} \boldsymbol{\alpha}^{2}\right],
$$

being $\boldsymbol{\alpha}=\exp (j 2 \pi / 3)$, and the dc supply voltage $2 V_{d c}$, according to Fig. 5.9.

For the three-level inverter, the output voltage can be similarly written by introducing the switching states $S_{k}=[-1,0,1]$ leading to

$$
\boldsymbol{v}=\frac{2}{3} V_{d c}\left[S_{1}+S_{2} \boldsymbol{\alpha}+S_{3} \boldsymbol{\alpha}^{2}\right] .
$$

Due to the symmetry among the three phases in the considered case of sinusoidal balanced currents, only the first phase is examined in the following analysis. In terms of space vectors, the variables of the first phase are given by the projection of the corresponding space vectors on the real axes. In particular, 
if the reference voltage is within the modulation limits, i.e. the reference space vector $v^{*}$ lies within the outer hexagon, the average output voltage is given by

$$
\bar{v}\left(T_{s}\right)=v^{*}=\operatorname{Re}\left\{v^{*}\right\}=V^{*} \cos \vartheta=V_{d c} m \cos \vartheta .
$$

The instantaneous output phase voltage $v(t)$ can be expressed by the switching states. For the first phase it can be calculated as the real part of the output voltage vectors (5.12) and (5.13), leading to

for two-level VSI: $\quad v=2 V_{d c}\left[S_{1}-\frac{1}{3}\left(S_{1}+S_{2}+S_{3}\right)\right]$,

and three-level VSI: $\quad v=V_{d c}\left[S_{1}-\frac{1}{3}\left(S_{1}+S_{2}+S_{3}\right)\right]$.

The alternating component of inverter output voltage of the first phase can be determined by introducing for two-level inverter (5.14) and (5.15) in (2.6), or for three-level inverter (5.14) and (5.16) in (2.6), leading to

for two-level VSI: $\tilde{v}(t)=2 V_{d c}\left[S_{1}-\frac{1}{3}\left(S_{1}+S_{2}+S_{3}\right)\right]-m V_{d c} \cos \vartheta$,

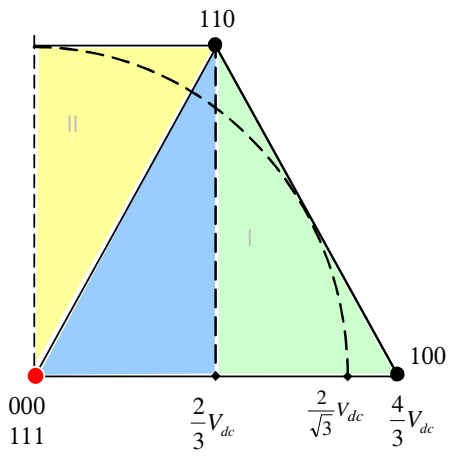

2-level VSI

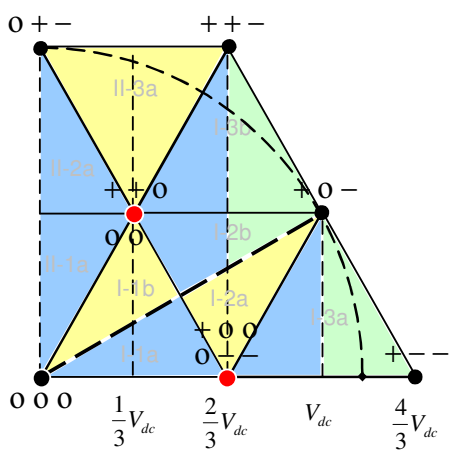

3-level VSI

Fig. 5.11. Output voltage diagram of two-level and three-level inverter in the first quadrant of $\alpha-\beta$ plane 
and three-level VSI: $\tilde{v}(t)=V_{d c}\left[S_{1}-\frac{1}{3}\left(S_{1}+S_{2}+S_{3}\right)\right]-m V_{d c} \cos \vartheta$

For the current ripple evaluation in the whole phase angle range $0<\vartheta<\pi / 2$ for both inverters, different cases corresponding to different sectors were considered, identified in Fig. 5. 11. The choice made for dc supply voltages leads to hexagons of equal size for two- and three-level inverters, corresponding to the same output voltage modulation limits. Additional subcases, also determined by the value of modulation index, are identified in Fig. 5.11 with different colored areas. Peak-to-peak current ripple has been evaluated on the basis of the instantaneous current ripple and its peak-to-peak value, (2.10) and (2.11), and by taking into account (5.17) for two-level inverter, and (5.18) for three-level inverter, following the procedures developed in previous chapters.

\subsubsection{Comparison of the peak-to-peak current ripple diagrams}

In order to show the behavior of the peak-to-peak current ripple amplitude in the fundamental period for all the considered cases, in Figs. 5.12 and 5.13 is represented the normalized function $r(m, \vartheta)$ defined by (5.7). Fig. 5.12 shows $r(\vartheta)$ for $m=1 / 3,2 / 3$, and 1 (from left to right), for two-, and three-level inverters (red and blue lines), respectively. According to Chapter 2, ripple in two-level inverters is shown to be almost linear with the modulation index and a simplified expression for maximum of peak-to-peak current ripple amplitude is obtained

$$
\tilde{i}_{p p}{ }^{\max } \cong \frac{V_{d c} T_{s}}{3.46 L} m, r^{\max } \cong 0.578 m
$$

From Fig. 5.12 can be noticed that the maximum current ripple amplitude for three-level inverters, blue line, is almost constant for intermediate modulation indexes, i.e., $m \approx[0.1-1]$, ranging around the value 0.2 , leading to the simplified expression

$$
\tilde{i}_{p p}{ }^{\max } \approx \frac{V_{d c} T_{s}}{10 L} \text { or } r^{\max } \approx 0.2 \text { in the approximate range } 0.1 \leq m \leq 1
$$



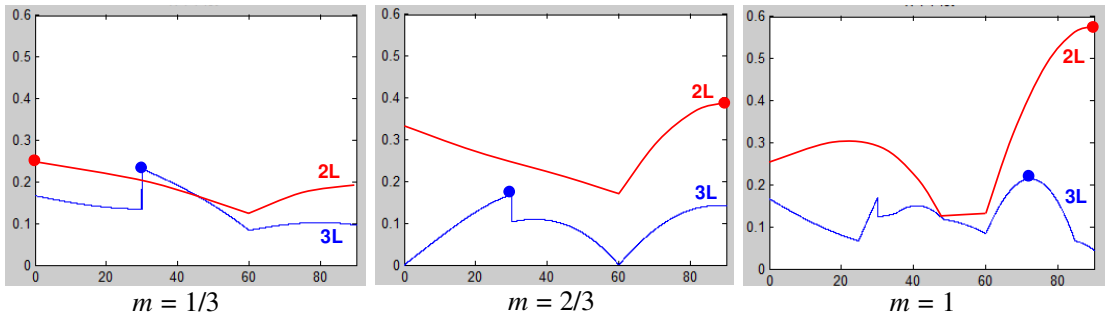

Fig. 5.12. Normalized peak-to-peak current ripple amplitude $r(m, \vartheta)$ for two-level and three-level VSIs in the range $\vartheta=\left[0,90^{\circ}\right]$ for different modulation indexes.

By considering the low modulation indexes $(0<m<1 / 3)$, we can notice that the maximum of the current ripple shows better behavior for the two-level inverter. For the higher modulation indexes the behavior of the three-level inverter is considerably better.

Fig. 5.13 shows the colored maps of $r(m, \vartheta)$ for the first quadrant within the modulation limits for two- and three-level inverters, respectively. For both two-level and three-level inverters can be noted that ripple amplitude is obviously zero for $m=0$, since the null configurations are the only applied. The same can be noted for the tips of the output voltage vectors, since the reference vector is almost perfectly synthesized and the alternating voltage goes to zero. Even though are kept the same colors for both cases, the normalized ripple of the two-level inverter is the double than for three-level inverter, since there is double dc voltage applied. In the map of three-level inverter the part of the hexagon of the two-level inverter can be identified, with

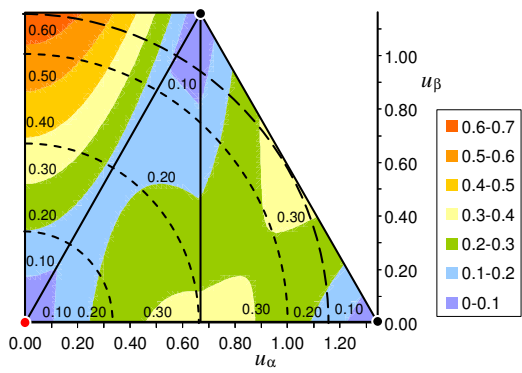

two-level VSI

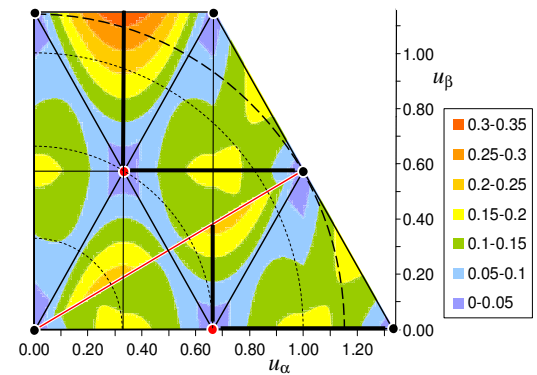

three-level VSI

Fig. 5.13. Map of the normalized peak-to-peak current ripple amplitude $r(m, \vartheta)$ for two-level and three-level VSIs, respectively. 
selected bolded areas matching the ripple distribution. The discontinuity of the ripple across the borders of pivot sector can be noted, due to the pivot sector change (red line).

On Fig. 5.14 the average values of the normalized current ripple as the function of the modulation index are shown. Three different cases are considered, three-level and two-level inverter with same output voltage range, i.e. three-level supplied with $V_{d c}$ and two-level with $2 V_{d c}$, and two-level inverter supplied with $V_{d c}$. It is clear, also from the previous observations, that the two-level inverter supplied with $2 V_{d c}$ has almost the double of the normalized ripple average compared to the three-level inverter, except for the low modulation indexes. On the other hand, the two-level inverter supplied with $V_{d c}$ shows the better behavior for the low modulation indexes, approximately for $m<1 / 3$. This can be explained with the reference to the Fig. 5.11. For the modulation indexes $m<1 / 3$, the nearest pivot vector is the zero vector, that is the one used in the modulation of the two-level inverter. In the case of modulation of the three-level inverter, one of the six pivot vectors, $\boldsymbol{v}_{\mathrm{p} 1}$ $\boldsymbol{v}_{\mathrm{p} 6}$, is used, depending where reference voltage lies, also if the voltage reference is more close to zero.

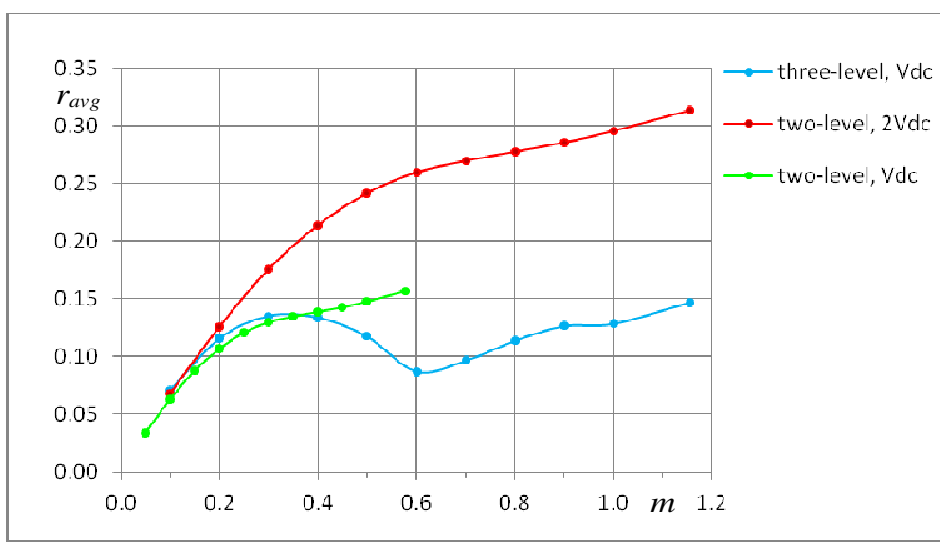

Fig. 5.14. The average of the normalized peak-to-peak current ripple amplitude as function of modulation index, for two-level inverter supplied by $V_{d c}$ and $2 V_{d c}$, and three-level inverter by $V_{d c}$. 


\subsubsection{Numerical results}

In order to verify the theoretical developments shown in previous section, circuit simulations are carried out by Sim-PowerSystems of Matlab considering two-level inverter and three-level cascaded H-bridge inverter with ideal switches connected to balanced RL load: $R=20 \Omega$ and $L=3 \mathrm{mH}$. In all simulations fundamental frequency $f$ is set to $50 \mathrm{~Hz}$, the switching frequency $1 / T_{s}$ is $6 \mathrm{kHz}$, and the dc voltage supply $V_{d c}=300 \mathrm{~V}$. A centered symmetrical carrier-based PWM technique is considered for both inverters. In the simulations instantaneous current ripple is calculated as the difference between the instantaneous and fundamental current component as in (5.11).

The first phase is selected for further analysis and different values of $m$ are investigated $(1 / 3,2 / 3$, and 1$)$.

Figs. 5.15a, 5.16a, and 5.17a (left columns) show the comparison between the instantaneous current ripple $\tilde{i}$ obtained in simulations by (5.11) (pink trace), and the half of peak-to-peak current ripple obtained by the analytical developments, $\tilde{i}_{p p} / 2$ (blue traces), for two-level and three-level inverters in one fundamental period. The results are obtained with different modulation indexes.

The corresponding Figs. 5.15b, 5.16b, and 5.17b (right column) show the comparison between the simulated instantaneous output current (pink trace) and calculated upper/lower current envelope (blue trace), for different modulation indexes.

The considered modulation indexes cover some of the possible sub-cases, i.e., colored regions in Fig. 5.11. Also the simulation results prove the conclusions made in previous section about the comparison of the two inverters. The agreement is good in the whole fundamental period, for all cases. 

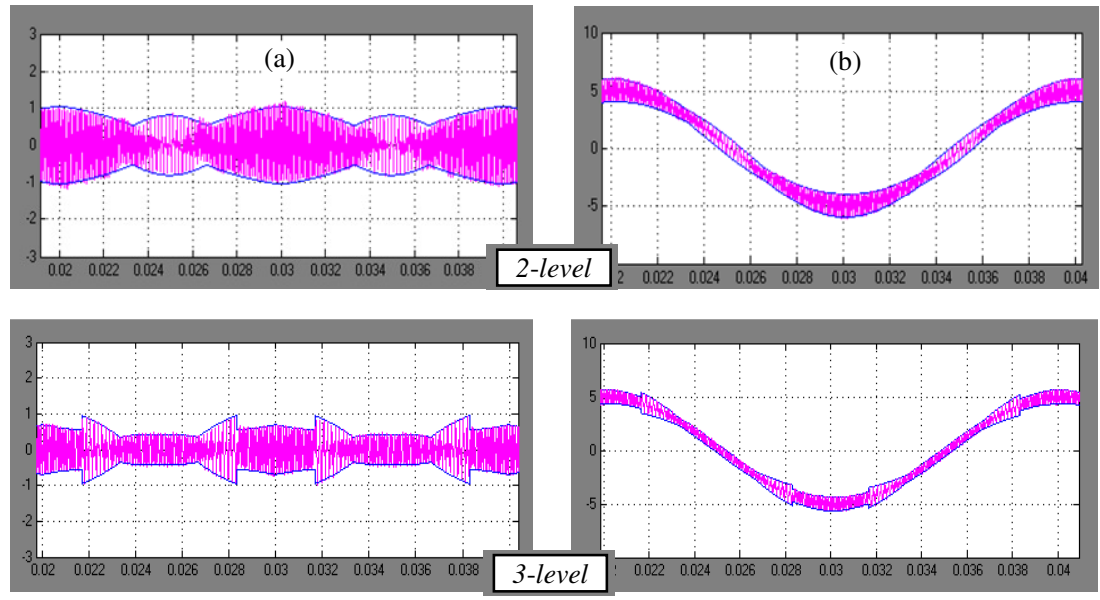

Fig. 5.15. Comparison between the simulation results (pink trace) and evaluated current ripple (blue trace) for $m=1 / 3$ : (a) simulated current ripple and evaluated peak-to-peak amplitude, (b) simulated instantaneous output current with calculated current envelopes.
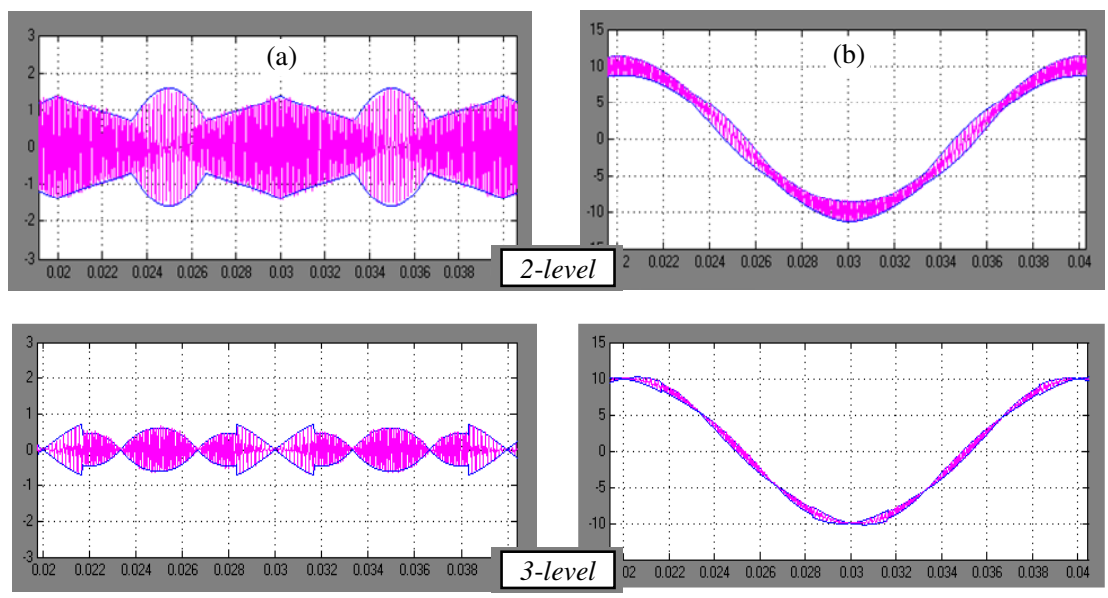

Fig. 5.16. Comparison between the simulation results (pink trace) and evaluated current ripple (blue trace) for $m=2 / 3$ : (a) simulated current ripple and evaluated peak-to-peak amplitude, (b) simulated instantaneous output current with calculated current envelopes. 

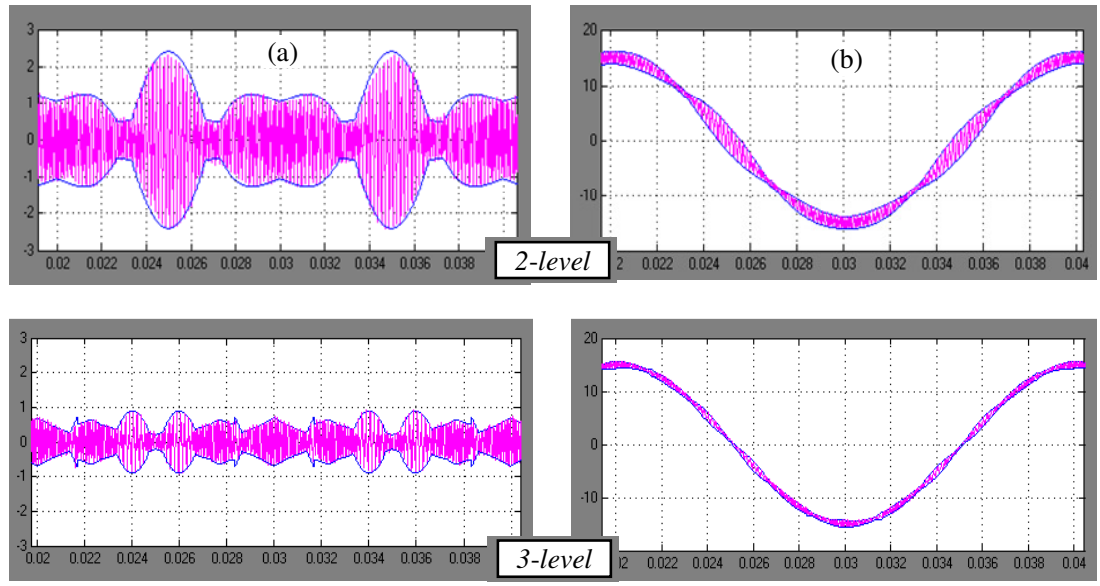

Fig. 5.17. Comparison between the simulation results (pink trace) and evaluated current ripple (blue trace) for $m=1$ : (a) simulated current ripple and evaluated peak-to-peak amplitude, (b) simulated instantaneous output current with calculated current envelopes.

\subsection{Authored papers}

- G. Grandi, J. Loncarski, C. Rossi, "Comparison of peak-to-peak current ripple amplitude in multiphase PWM voltage source inverters," In Proc. of $15^{\text {th }}$ IEEE Conf. on Power Electronics and Applications (EPE'13 ECCE Europe), Lille FR, 3-5 Sept 2013. DOI: 10.1109/EPE.2013.6634719 


\section{Examples of application}

The evaluation of the peak-to-peak output current ripple, in addition to the RMS of the output current ripple which has been studied in the literature, can be used in several applications. In particular, the knowledge of the peak-topeak current ripple distribution can be useful to determine the output voltage distortion due to the inverter dead-time in case of output currents with high ripple, by determining the multiple zero-crossing interval [1]. Another example of application is referred to the comparison of the band in hysteresis current controller and the average of the peak-to-peak amplitude in terms of the switching frequency [2]-[6]. Peak-to-peak current ripple evaluation can also be used in variable switching frequency PWM control architecture. In particular, switching frequency can be adapted in each switching period to satisfy certain ripple requirements, such as ripple peak or ripple RMS. By setting the maximum ripple peak following the requirements of the system, it is possible to lower the switching frequency in some regions where it is noted that ripple is lower than the maximum [4]. Furthermore, the peak-to-peak current ripple amplitude, in addition to the fundamental current component, is useful to determine the absolute current peak, affecting the thresholds of protection systems and the design of power components. The first two mentioned examples will be further developed in this chapter.

\section{1) Determination of the zero-crossing interval}

The example of the application where value of the peak-to-peak current ripple can used for the calculation of the current zero-crossing intervals is explained in more details. The dead-time effects are well known in the case when the sinusoidal output currents with single zero-crossing are considered. However, the studies can be extended also considering the case of the highripple output currents, with multiple zero-crossings. In general, dead-time effects can be determined on the basis of the sign of the output current. If the current is positive, part of the pole voltage pulse will be lost, depending on the amount of the dead-time. If the current is negative, the corresponding part of the pole voltage pulse will be gained. It can be simply be evaluated by the expression for the actual pole voltage of $k$-th leg $(k=1,2,3), e_{k}$, which can be 

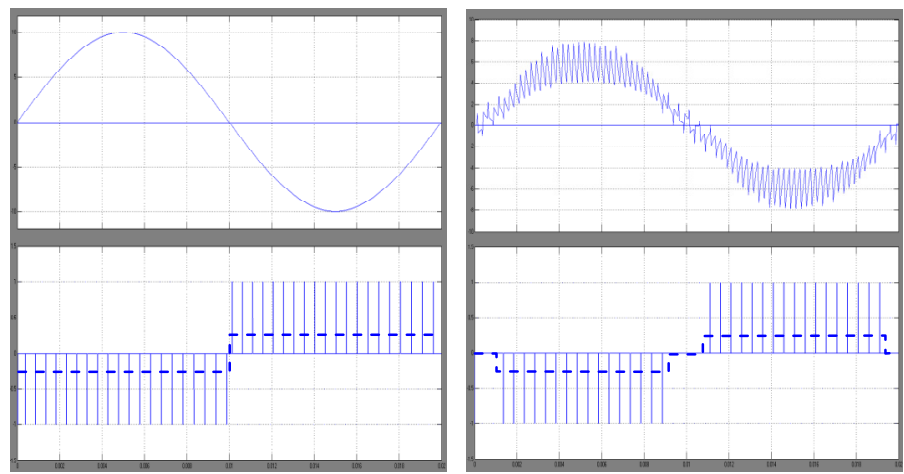

Fig. 6.1. Current $i_{k}$ (top) and dead-time pole voltage error (bottom) for three-phase VSI with currents without ripple (left) and with ripple (right)

calculated on the basis of the reference pole voltage, $e_{k}{ }^{*}$, and the direction of the phase current $i_{k}$, as

$$
e_{k}=e_{k}^{*}-\operatorname{sign}\left(i_{k}\right) \Delta V_{d},
$$

where $\Delta V_{d}$ is the averaged voltage contribution due to the dead-time $t_{d}$ over the switching period $T_{s}, \Delta V_{d}=t_{d} / T_{s} V_{d c}$. High output current ripple introduces multiple zero-crossings, leading to modified output voltage distortions.

The further analysis has been carried out in the case of the three-phase inverter with balanced RL load, but it can readily be extended also to multiphase inverters, as done in the case of dead-time effects for pure sinusoidal currents [7], [8]. If the output current is with ripple, the effect of dead-time is reduced, since during the zero-crossings the average of sign function of current is zero, so there is no effect on specific pulses of pole voltage at zero-crossings. Taking this into account, dead-time doesn't affect the width of the pole voltage pulse if the current turns from negative to positive during the voltage pulse. On Fig. 6.1 are shown the phase currents without and with ripple and the corresponding dead-time instantaneous voltage error (the dashed line is the average), obtained with simulation. In the case of sinusoidal current there is continuous dead-time effect. In the case when there is highripple current, around zero crossing there are no dead-time effects. The result when current changes sign from negative to positive during the pulse is that actual pulse is equal to reference one. On Fig. 6.2a is depicted the current with 


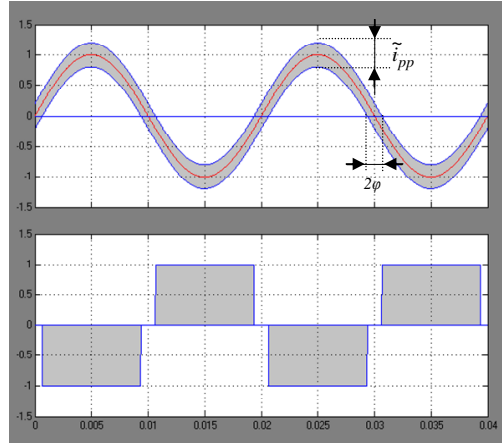

(a)

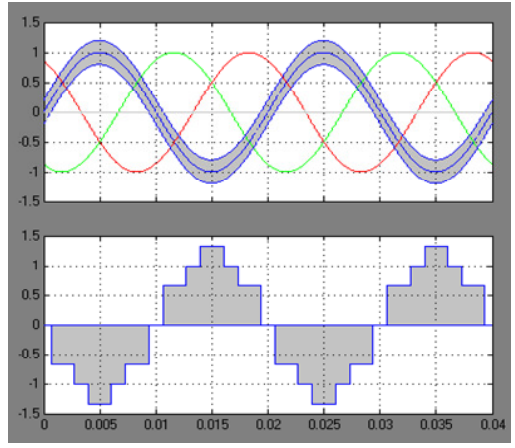

(b)

Fig. 6.2. (a) Current $i_{\mathrm{k}}$ with peak-to-peak envelope of ripple (top) with averaged pole voltage error, (b) Three-phase currents $i_{k}$ (top) and load voltage error $u_{1}$ (normalized with respect to $\Delta V_{d}$, bottom)

peak-to-peak envelope of ripple and averaged pole voltage error due to deadtime, taking into account the zero crossings. This effect on pole voltage can be summarized with a modified sign function $\underline{\operatorname{sign}}\left(i_{k}\right)$, having zero values corresponding to multiple zero-crossing interval of the current. Introducing this effect to the pole voltage, modified pole voltage is still calculated on the basis of (6.1), but taking into account the modified sign function, according to the direction of the phase current $i_{k}$ as

$$
e_{k}=e_{k}^{*}-\underline{\operatorname{sign}}\left(i_{k}\right) \Delta V_{d} .
$$

The modified effects of the dead-time on the load voltage can be evaluated starting from the expression for the actual load voltage $v_{k}$ expressed by pole voltages

$$
v_{k}=e_{k}-\left(e_{1}+e_{2}+e_{3}\right) / 3 .
$$

Introducing in (6.3) the modified pole voltages (6.2) leads to

$$
v_{k}=v_{k}^{*}+u_{k},
$$

being $v_{k}^{*}$ the reference load voltage

$$
v_{k}^{*}=e_{k}^{*}-\left(e_{1}^{*}+e_{2}^{*}+e_{3}^{*}\right) / 3,
$$

and $u_{k}$ the modified average load voltage error introduced by dead-time 

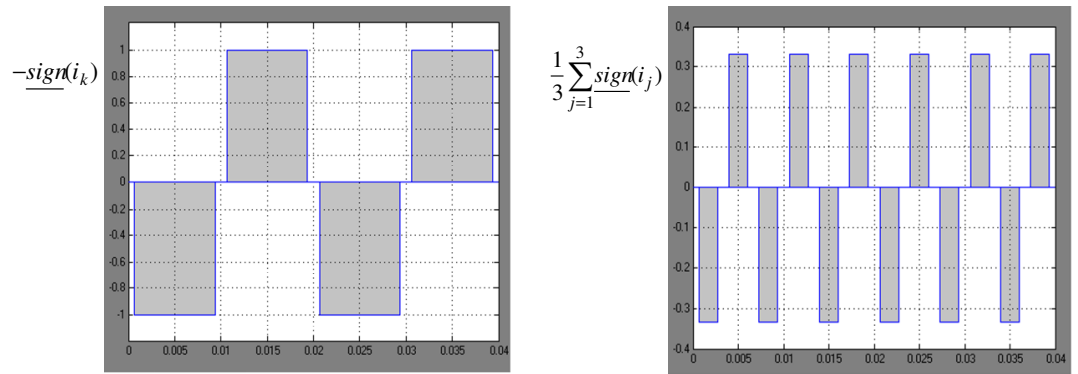

Fig. 6.3. Waveforms of the two contributions to the voltage error $u_{k}$ normalized with respect to $\Delta V_{d}$

$$
\left.u_{k}=\Delta V_{d}\left\{\underline{-\operatorname{sign}}\left(i_{k}\right)+\underline{\operatorname{sign}}\left(i_{1}\right)+\underline{\operatorname{sign}}\left(i_{2}\right)+\underline{\operatorname{sign}}\left(i_{3}\right)\right] / 3\right\} .
$$

The waveform of $u_{k}$ depicted in Fig. 6.2b, can be seen as the sum of two contributions: the sign of the $k$-th leg current, $-\underline{\operatorname{sign}}\left(i_{k}\right)$, and average of signs of all output currents $1 / 3 \sum \underline{\operatorname{sign}}\left(i_{k}\right)$, considering modified sign functions. The two contributions are represented in Fig. 6.3. The first contribution corresponds to unity square wave signal with fundamental period $T$, but modified with taking into account the zero values due to zero crossings. The second one corresponds to a square wave signal, also modified, with amplitude $1 / n$ and period $T / n$, being $n$ the number of phases, in this case $n=3$.

The Fourier development of the load voltage error (6.6) can be obtained as a combination based on these two signals, leading to

$$
\begin{aligned}
& u_{k}=\Delta V_{d} \frac{4}{\pi}\left\{-\cos \varphi \sin \left[\omega_{0} t-(k-1) \frac{2 \pi}{3}\right]-\frac{1}{3} \cos (3 \varphi) \sin \left[3\left(\omega_{0} t-(k-1) \frac{2 \pi}{3}\right)\right]-\right. \\
& -\frac{1}{5} \cos (5 \varphi) \sin \left[5\left(\omega_{0} t-(k-1) \frac{2 \pi}{3}\right)\right]-\ldots+\frac{1}{3} \cos (3 \varphi) \sin \left[3\left(\omega_{0} t-(k-1) \frac{2 \pi}{3}\right)\right]+ \\
& \left.+\frac{1}{9} \cos (9 \varphi) \sin \left[9\left(\omega_{0} t-(k-1) \frac{2 \pi}{3}\right)\right]+\frac{1}{15} \cos (15 \varphi) \sin \left[15\left(\omega_{0} t-(k-1) \frac{2 \pi}{3}\right)\right]+\ldots\right\}
\end{aligned}
$$


where $\omega_{0}=2 \pi / T$ is the fundamental frequency and $\varphi$ is the angle corresponding to half of the multiple zero crossing period of the current, as represented in Fig. $6.2 \mathrm{a}$.

From (6.7) can be noticed that all harmonic components of the second signal completely erase with corresponding harmonics of the first signal, leading to the following simplified expression

$$
u_{k}=-\Delta V_{d} \frac{4}{\pi} \sum_{h=1}^{\infty} \frac{1}{h} \cos (h \varphi) \sin \left[h\left(\omega_{o} t-(k-1) \frac{2 \pi}{3}\right)\right] .
$$

The amplitude of harmonics is calculated according to (6.8) as

$$
U_{h}=\frac{4}{\pi} \frac{1}{h} \Delta V_{d} \cos (h \varphi),(h \text { odd integer not multiple of } n) .
$$

The amplitude of fundamental voltage error is given by (6.9) having $h=1$ is

$$
U_{1}=\frac{4}{\pi} \Delta V_{d} \cos (\varphi) .
$$

In order to verify what is the effect of dead-time on output voltages, i.e. amplitude of the harmonics, multiple zero-crossing time interval (angle $\varphi$ ) of output currents should be determined. According to Fig. 6.2a, the angle corresponding to zero crossings of the current can be evaluated on the basis of the half of the peak-to-peak current envelope and fundamental of the current amplitude as

$$
\varphi=\arcsin \left(\frac{\tilde{i}_{p p} / 2}{I_{\text {fund }}}\right) .
$$

The peak-to-peak output current ripple should be calculated for the specific case $\left(\tilde{i}_{p p} / 2\right.$ during the zero crossing of the current, i.e. $\vartheta=\phi-\pi / 2$, being $\phi$ the load power factor angle), as introduced in Chapter 2 for the three-phase two-level inverters. In this way, knowing the peak-to-peak current ripple amplitude, the angle corresponding to the zero crossing of the current $\varphi$ can be determined, and furthermore also the effect of the dead-time on the output voltages. 


\section{1) Comparison with the hysteresis current controller}

The peak-to-peak current ripple envelope can be used in comparison with the constant band hysteresis controllers. By determining the average of the peak-to-peak ripple envelope and setting it as the band in hysteresis controller, the two frequencies can be compared.

This is further examined with the simulations implemented by Simulink, Matlab. The three-phase inverter connected to sinusoidal voltage sources by RL impedance is considered in the simulations, having $R=5 \Omega$ and $L=20$ $\mathrm{mH}$. One simulation is done with implemented optimal carrier-based PWM, and the other with hysteresis current controller. In both simulations fundamental frequency is set to $50 \mathrm{~Hz}$, and the dc voltage supply $V_{d c}$ is $600 \mathrm{~V}$. The fundamental of the output current was kept at constant value by adjusting the voltage sources. In the simulation with PWM, the switching frequency $1 / T_{s}$ was set to $2.1 \mathrm{kHz}$. Different modulation indexes have been considered (1/6, $1 / 5,1 / 4,1 / 3,2 / 5,1 / 2$, and $1 / \sqrt{ } 3=m_{\max }$ ).

In the simulation with PWM the upper and lower peak-to-peak current ripple envelope, $\tilde{i}_{p p} / 2$, is calculated as explained in Chapter 2 for three-phase two-level inverters. In the cases of different modulation indexes, the different values for the average of the ripple envelope have been calculated, as shown in Table I. In further step, this average values were set as the upper and lower constant band in the simulation with hysteresis current controller, i.e. $\pm \tilde{i}_{p p_{-} a v g} / 2$.

Table I: Switching frequencies with hysteresis current controller and PWM

\begin{tabular}{|c|c|c|c|c|c|c|c|}
\hline \multicolumn{2}{|c|}{$m$} & \multicolumn{5}{c|}{ Switching frequency, $f_{c}[\mathrm{kHz}]$} \\
\hline $1 / 6$ & 0.17 & 0.34 & 3.4 & 3.3 & 3.5 & 3.4 & 2.1 \\
\hline $1 / 5$ & 0.20 & 0.38 & 2.6 & 2.7 & 2.9 & 2.7 & 2.1 \\
\hline $1 / 4$ & 0.25 & 0.43 & 2.7 & 2.9 & 3.0 & 2.9 & 2.1 \\
\hline $1 / 3$ & 0.33 & 0.48 & 2.7 & 2.6 & 3.0 & 2.8 & 2.1 \\
\hline $2 / 5$ & 0.40 & 0.50 & 2.6 & 2.6 & 2.4 & 2.5 & 2.1 \\
\hline $1 / 2$ & 0.50 & 0.53 & 1.7 & 1.9 & 1.9 & 1.8 & 2.1 \\
\hline $1 / \sqrt{ } 3$ & 0.58 & 0.56 & 1.2 & 1.2 & 1.2 & 1.2 & 2.1 \\
\hline
\end{tabular}


The gate signals of the two IGBTs for all three legs were processed with the implemented pulse counter, in order to determine the switching frequency. In Table I the results of all the frequencies are shown for all the considered cases.

In Fig. 6.4 is shown the comparison of the frequencies obtained with PWM and hysteresis current controller. The PWM shows better behavior in wide range of modulation indexes, i.e. approximately $0.2 \leq m \leq 0.46$. For the modulation indexes $m>0.46$, the hysteresis current controller has the frequency that is lower.

In Figs. 6.5, 6.6, 6.7, and 6.8 the results obtained with simulations are shown, with some of the considered modulation indexes. In Figs. 6.5a-6.8a is shown the simulated ripple with PWM (up) with its calculated upper and lower envelope. The average value of the half peak-to-peak envelope is emphasized in red color. The ripple simulated with hysteresis current controller (down) is shown with its constant bands. Since in the hysteresis current controller there are three independent controllers, the comparator state change in one phase influence the voltage in the other phase. The consequence of that is that instantaneous error can reach double the value of the hysteresis band [9], as can be observed in the figures.

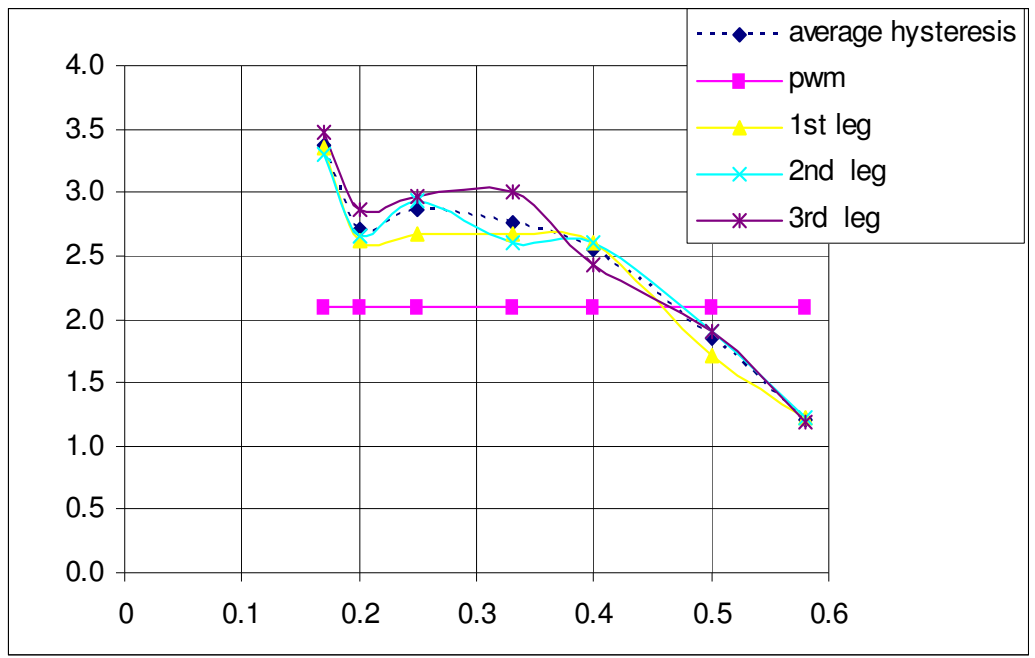

Fig. 6.4. Comparison of the frequencies obtained with PWM and hysteresis current controller. 
In Figs. 6.5b-6.8b are shown the instantaneous currents with PWM modulation (up) and hysteresis current controller (down). Instantaneous current with PWM is shown with calculated upper and lower ripple envelopes. The corresponding current simulated with hysteresis controller is show with its constant upper and lower bands. 

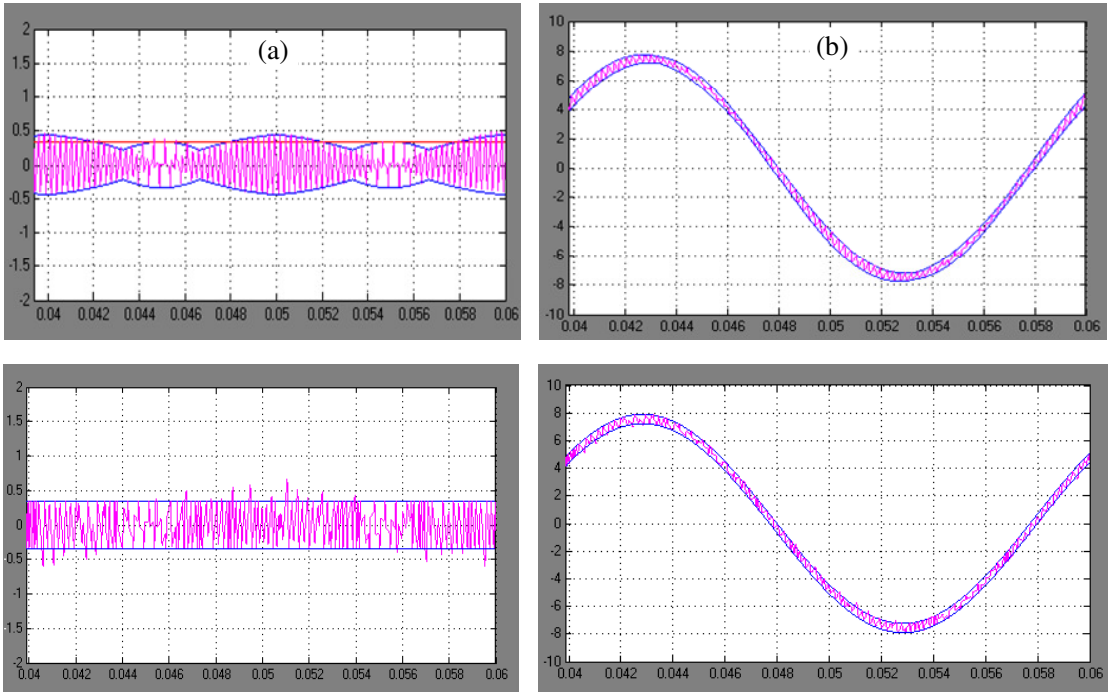

Fig. 6.5. Simulations with PWM (up) and hysteresis current controller (down), $m=1 / 6$ : (a) output current ripples with calculated/constant envelopes/band (blue color), (b) instantaneous output current with calculated/constant upper and lower envelope/band.
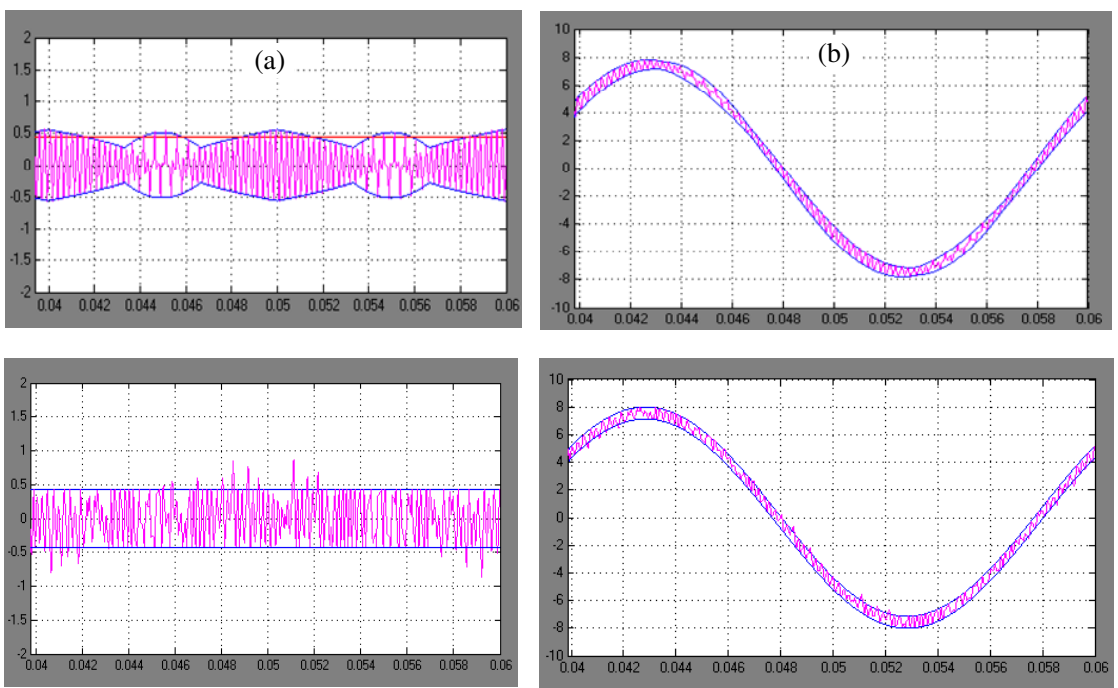

Fig. 6.6. Simulations with PWM (up) and hysteresis current controller (down), $m=1 / 4$ : (a) output current ripples with calculated/constant envelopes/band (blue color), (b) instantaneous output current with calculated/constant upper and lower envelope/band. 

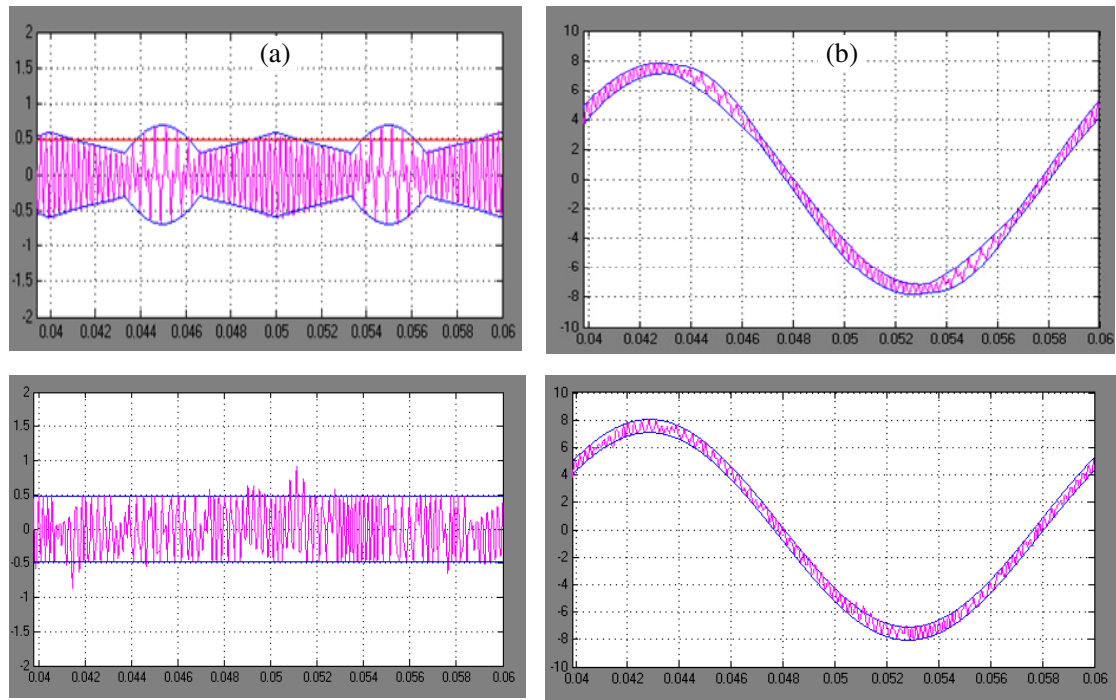

Fig. 6.7. Simulations with PWM (up) and hysteresis current controller (down), $m=1 / 3$ : (a) output current ripples with calculated/constant envelopes/band (blue color), (b) instantaneous output current with calculated/constant upper and lower envelope/band.
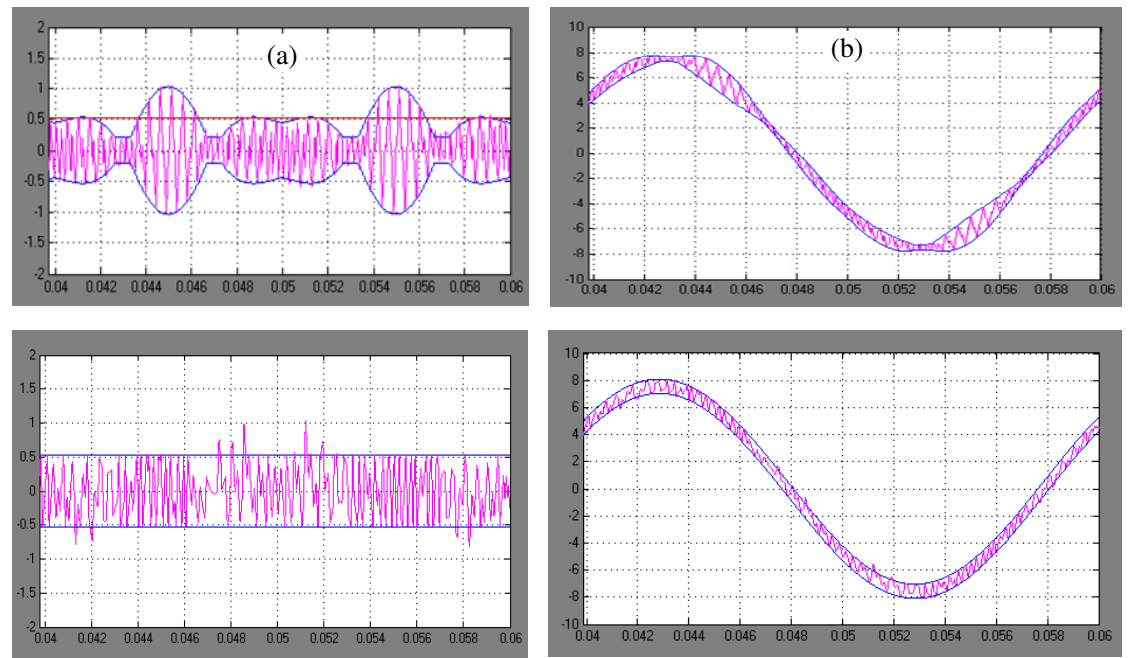

Fig. 6.8. Simulations with PWM (up) and hysteresis current controller (down), $m=1 / 2$ : (a) output current ripples with calculated/constant envelopes/band (blue color), (b) instantaneous output current with calculated/constant upper and lower envelope/band. 


\subsection{References}

[1] G. Grandi, J. Loncarski, R. Seebacher, "Effects of current ripple on dead-time analysis of three-phase inverters, " In Proc. of IEEE Energy Conference (ENERGYCON), Florence IT, 9-12 Sept. 2012, pp. 207212 .

[2] C.N.M. Ho, V.S.P. Cheung, H.S.H. Chung, "Constant-frequency hysteresis current control of grid-connected VSI without bandwidth control, "IEEE Trans. on Power Electronics, vol. 24, no. 11, pp. 2484 2495, Nov. 2009.

[3] D.G. Holmes, R. Davoodnezhad, B.P. McGrath, "An improved threephase variable-band hysteresis current regulator, "IEEE Trans. on Power Electronics, vol. 28, no. 1, pp. 441-450, Jan. 2013.

[4] D. Jiang, F. Wang, "Variable switching frequency PWM for three-phase converters based on current ripple prediction, " IEEE Trans. on Power Electronics, vol. 28, no. 11, pp. 4951-4961, Nov. 2013.

[5] S. Srikanthan, M.K. Mishra, R.K.V. Rao, "Improved hysteresis current control of three-level inverter for distribution static compensator application," IET Power Electronics, vol. 2, no. 5, pp. 517 - 526, Sept. 2009.

[6] A. Shukla, A. Ghosh, A. Joshi, "Hysteresis modulation of multilevel inverters, " IEEE Trans. on Power Electronics, vol. 26, no. 5, pp. 1396 1409, May 2011.

[7] G. Grandi, J. Loncarski, "Analysis of dead-time effects in multi-phase voltage sourse inverters," Proc. of IET Power Electronics, Machines and Drives (PEMD), Bristol, UK, CD-ROM paper 0223, 2012, DOI: 10.1049/cp.2012.0170.

[8] G. Grandi, J. Loncarski, "Space Vector Analysis of Dead-Time Voltage Distortion in Multiphase Inverters," Proc. of $15^{\text {th }}$ Power Electronics and Motion Control Conference (EPE-PEMC), Novi Sad, RS, Sept. 4-6, 2012, DOI: 10.1109/EPEPEMC.2012.6397342.

[9] M. Kazimierkowski, L. Malesani, "Current control techniques for threephase voltage-sourcc converters: A survey," IEEE Transactions on Industrial Electronics, vo1. 45, no. 5, pp. 691-703, October 1998. 


\section{Conclusion and future work}

\subsection{Conclusion}

This thesis deals with the analysis of the peak-to-peak output current ripple amplitude in multiphase and multilevel inverters with reference to centered symmetrical PWM, that is optimal modulation in terms of minimum output current RMS.

The current ripple was defined by observing the output voltage waveforms for all the different cases indentified in the whole modulating range with the considered PWM strategy. The analysis has been carried out in first quadrant of the output voltage vector, exploiting the quarter-wave symmetry for the extension to the whole fundamental period. In all the considered cases, the current ripple is defined with reference to simplified RLE circuit load, which can represent, in most of cases, both grid-connected systems and motor loads.

The expression of the peak-to-peak current ripple amplitude has been determined as a function of the modulation index, and an effective normalization has been introduced to better emphasize the ripple behavior and to summarize it in some diagrams and maps.

Simplified expressions to determine the maximum peak-to-peak ripple amplitude have been proposed for both multiphase and multilevel inverters. The peak-to-peak ripple is compared for the different cases of phase and level numbers, and some application examples are given for three-phase systems. Both simulation and experimental results are presented to prove the analytical developments. The agreement among numerical, experimental, and analytical results is good for all the relevant considered cases, proving the validity of the proposed approach. 


\subsection{Future work}

The material presented in this thesis, in spite the significant new knowledge has been developed, leaves large space for further research work. Some of them are listed here, as possible directions for future work:

- investigation of the peak-to-peak output current ripple in multilevel inverters for higher number of levels;

- investigation of the variable switching frequency techniques able to produce optimal switching patterns in terms of absolute peak-to-peak maximum and RMS ripple limitations;

- correlation between peak-to-peak amplitude and current ripple RMS;

- since the developments have been carried out specifically for symmetrical centered PWM, they can be similarly extended to discontinuous and/or asymmetrical modulations. 